\title{
QUANTUM ALGEBRAS AND SYMPLECTIC REFLECTION ALGEBRAS FOR WREATH PRODUCTS
}

\author{
NICOLAS GUAY
}

\begin{abstract}
To a finite subgroup $\Gamma$ of $S L_{2}(\mathbb{C})$, we associate a new family of quantum algebras which are related to symplectic reflection algebras for wreath products $S_{l} \succ \Gamma$ via a functor of Schur-Weyl type. We explain that they are deformations of matrix algebras over rank-one symplectic reflection algebras for $\Gamma$ and construct for them a PBW basis. When $\Gamma$ is a cyclic group, we are able to give more information about their structure and to relate them to Yangians.
\end{abstract}

\section{INTRODUCTION}

The theory of symplectic reflection algebras was introduced a few years ago in the seminal paper EtGi of P. Etingof and V. Ginzburg. Since then, applications have been found in representation theory and in algebraic geometry; see, e.g., Bo, EGGO, GoSt, GoSm]. One important example of such algebras is given by the rational Cherednik algebras [Ch2, GGOR, BEG associated to a complex reflection group $W$ acting on the symplectic vector space $\mathfrak{h} \oplus \mathfrak{h}^{*}, \mathfrak{h}$ being its reflection representation. A large class of symplectic reflection algebras are those associated to wreath products $\Gamma_{l}=S_{l} \succ \Gamma=\Gamma^{\times l} \rtimes S_{l}$ for $\Gamma$ a finite subgroup of $S L_{2}(\mathbb{C})$.

In this paper, we introduce a new family of quantum algebras that we call $\Gamma$ deformed double current algebras $(\Gamma-\mathrm{DDCA})$. They are flat deformations of the enveloping algebra of an enlargement of $\widehat{\mathfrak{s l}}_{n}(\mathbb{C}[u, v] \rtimes \Gamma)$, the universal central extension of $\mathfrak{s l}_{n}(\mathbb{C}[u, v] \rtimes \Gamma)$. They can also be viewed as flat deformations of $\mathfrak{U g l}_{n}\left(A_{1} \rtimes \Gamma\right)$ where $A_{1}$ is the first Weyl algebra. We construct a PBW basis for $\Gamma$-DDCA by using a Schur-Weyl functor which relates them to symplectic reflection algebras for $\Gamma_{l}$. When $\Gamma=\mathbb{Z} / r \mathbb{Z}$, we are able to give a second definition of $\Gamma$-DDCA by realizing them as certain subalgebras of a cyclic version of affine Yangians.

One can consider the general problem of studying spaces of maps $X \longrightarrow \mathfrak{g}$ from an algebraic variety to a semisimple Lie algebra $\mathfrak{g}$. When $X$ is smooth and of dimension one, this leads to current Lie algebras $\mathfrak{g} \otimes_{\mathbb{C}} \mathbb{C}[u]$, loop algebras $\mathfrak{g} \otimes_{\mathbb{C}} \mathbb{C}\left[u, u^{-1}\right]$ and their universal central extensions, the affine Lie algebras. When $X$ has dimension two, the most natural case to consider is the two-dimensional torus $X=\mathbb{C}^{\times} \times \mathbb{C}^{\times}$, but two simpler cases are $X=\mathbb{C} \times \mathbb{C}^{\times}$and $X=\mathbb{C}^{2}$. Quantizations of the corresponding enveloping algebras are known as quantum toroidal algeras [GKV], affine Yangians and deformed double current algebras Gu2, respectively. We may also consider singular varieties, and one of the simplest example is a Kleinian singularity $\mathbb{C}^{2} / \Gamma$. We are thus led to consider the Lie algebra $\mathfrak{g} \otimes_{\mathbb{C}} \mathbb{C}[u, v]^{\Gamma}$ and its universal

Received by the editors October 19, 2007 and, in revised form, September 29, 2009. 2010 Mathematics Subject Classification. Primary 17B37; Secondary 20 C08.

(C)2010 American Mathematical Society 
central extension. We can also follow one of the main ideas in [EtGi] and replace the ring of invariants $\mathbb{C}[u, v]^{\Gamma}$ by the smash-product $\mathbb{C}[u, v] \rtimes \Gamma$ since it is believed by ring theorists that the latter encodes more the geometry of the quotient $\mathbb{C}^{2} / \Gamma$ and of its (minimal) resolution of singularities than the former. This is another motivation for studying $\Gamma$-DDCA.

The representation theory of quantum toroidal algebras was studied in $\mathrm{He} 1, \mathrm{He} 2$, VaVa1, VaVa2 and, via geometric methods, in VaVa3. We hope that understanding the representations of $\Gamma$-DDCA will eventually lead to a better understanding of quantum toroidal algebras and affine Yangians, but we will also exhibit new phenomena which do not occur for these two types of algebras.

After recalling the definition of symplectic reflection algebras for wreath products, we devote two sections to the Lie algebras $\mathfrak{s l}_{n}(\mathbb{C}[u, v])$ and $\widehat{\mathfrak{s l}}_{n}(\mathbb{C}[u, v] \rtimes \Gamma)$, giving presentations in terms of families of generators and relations which are useful later on. The main idea is to obtain presentations with only finitely many generators and relations of low degree. The principal results here are Lemma 3.5 and Lemma 4.1. The latter is a modified version of a theorem of C. Kassel and J.L. Loday KaLo which is useful for our purposes. We also mention some results pertaining to the first cyclic homology group of the smash product $\mathbb{C}[u, v] \rtimes \Gamma$ since this space gives the center of the universal central extension $\widehat{\mathfrak{s l}}_{n}(\mathbb{C}[u, v] \rtimes \Gamma)$. The following section is simply devoted to defining the $\Gamma$-deformed double current algebras $\mathrm{D}_{\beta, \mathbf{b}}^{n}$. Section 6 contains one of the main results of this paper: we explain how to extend the classical Schur-Weyl functor to the double affine setup and when it yields an equivalence of certain categories of modules; see Theorem 6.1. When $\lambda=0$, the $\Gamma$-deformed double current algebras are enveloping algebras of Lie algebras closely related to $\mathfrak{s l}_{n}$ with entries in a rank-one symplectic reflection algebra for $\Gamma$ : this is the content of section 7 Section 8 contains our second main theorem: we prove that the associated graded ring of $\mathrm{D}_{\beta \mathbf{b}}^{n}$ is isomorphic to the undeformed ring $\mathrm{D}_{\beta=0, \mathbf{b}=\mathbf{0}}^{n}$, whence the name PBW property by analogy with the classical Poincaré-Birkhoff-Witt theorem.

The second half of the paper (all of section 9) is devoted to the special case $\Gamma=\mathbb{Z} / d \mathbb{Z}$. The symplectic reflection algebras for the wreath product $(\mathbb{Z} / d \mathbb{Z})^{\times l} \rtimes$ $S_{l}$ are rational Cherednik algebras, so they afford a $\mathbb{Z}$-grading. This explains in part why we can obtain more results in this specific case. We start by studying certain degenerate affine Hecke algebras associated to $\Gamma_{l}$ and then extend the results of Gu1 to the double affine trigonometric setting where we have a functor of Schur-Weyl type (see Theorem 9.1) between modules for a localization of a rational Cherednik algebra for $\Gamma_{l}$ and a certain algebra which turns out to be isomorphic to a Yangian for $\mathfrak{s l}_{n d}$ (see Corollary 9.3). The main goal of section 9 is reached in subsection 9.3 where we prove that deformed double current algebras for $\mathbb{Z} / d \mathbb{Z}$ can be realized as subalgebras of certain loop Yangians: see Theorem 9.3. This provides another set of generators, which might be convenient in the study of representations.

Throughout this paper, we will assume that $n \geq 4$ : analogous results most probably hold for $n=2,3$, but some definitions may involve more complicated relations and certain proofs would have to be modified accordingly. 
Acknowledgments. The author gratefully acknowledges the financial support of the Ministère français de l'Enseignement supérieur et de la Recherche and would like to thank David Hernandez and the Laboratoire de Mathématiques de l'Université de Versailles-St-Quentin-en-Yvelines for their hospitality while this paper was written. The author is now supported by an NSERC Discovery Grant.

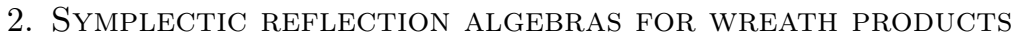

Isomorphism classes of finite subgroups of $S L_{2}(\mathbb{C})$ are known to be in bijective correspondence with affine Dynkin diagrams of type A,D,E via the McKay correspondence. We will denote by $\Gamma$ such a subgroup. For instance, the group corresponding to the Dynkin diagram of type $\widehat{A}_{r-1}$ is the cyclic group $\Gamma=\mathbb{Z} / r \mathbb{Z}$. In this section, we recall a few definitions and facts about symplectic reflection algebras for the wreath product $\Gamma_{l}=S_{l}\left\langle\Gamma\right.$ of $\Gamma$ with the symmetric group $S_{l}$. Let $\omega$ be a nondegenerate symplectic form on $U \cong \mathbb{C}^{2}$ and choose a basis $\{x, y\}$ of $U$ such that $\omega(x, y)=1$. We will denote by $\left\{x_{i}, y_{i}\right\}$ the same basis of $\mathbb{C}^{2}$, this time viewed as the $i^{\text {th }}$ direct summand of $U^{\oplus l}$. Note that $\Gamma_{l}$ acts on $U^{\oplus l}$.

The definition of a symplectic reflection algebra depends on two parameters: $t \in \mathbb{C}$ and $\mathbf{c}=\kappa \cdot \mathrm{id}+\sum_{\gamma \in \Gamma \backslash\{\mathrm{id}\}} c_{\gamma} \gamma \in \mathrm{Z} \Gamma$, which is an element in the center $\mathrm{Z} \Gamma$ of $\mathbb{C}[\Gamma]$. We have adapted the definition of the symplectic reflection algebra $\mathrm{H}_{t, \mathbf{c}}\left(\Gamma_{l}\right)$ from GaGi. For $\gamma \in \Gamma$, we write $\gamma_{i}$ for (id, .., id, $\gamma$, id, $\ldots$, id $) \in \Gamma_{l}$, where $\gamma$ is in the $i^{\text {th }}$ position.

Definition 2.1. The symplectic reflection algebra $\mathrm{H}_{t, \mathbf{c}}\left(\Gamma_{l}\right)$ is defined as the algebra generated by the two sets of pairwise commuting elements $x_{1}, \ldots, x_{l}, y_{1}, \ldots, y_{l}$ and by $\tau \in \Gamma_{l}$ with the relations:

$$
\begin{gathered}
\tau \cdot x_{i} \cdot \tau^{-1}=\tau\left(x_{i}\right), \quad \tau \cdot y_{i} \cdot \tau^{-1}=\tau\left(y_{i}\right), i=1, \ldots, l, \forall \tau \in \Gamma_{l}, \\
{\left[x_{i}, y_{i}\right]=t+\frac{\kappa}{2} \sum_{\substack{j=1 \\
j \neq i}}^{l} \sum_{\gamma \in \Gamma} \sigma_{i j} \gamma_{i} \gamma_{j}^{-1}+\sum_{\gamma \in \Gamma \backslash\{\mathrm{id}\}} c_{\gamma} \gamma_{i}, i=1, \ldots, l,}
\end{gathered}
$$

where $\sigma_{i j} \in S_{l}$ is the permutation $i \leftrightarrow j$. For $i \neq j$ and any $w^{1}, w^{2} \in U, w_{i}^{j} \in$ $\operatorname{span}\left\{x_{i}, y_{i}\right\}$ :

$$
\left[w_{i}^{1}, w_{j}^{2}\right]=-\frac{\kappa}{2} \sum_{\gamma \in \Gamma} \omega\left(\gamma\left(w^{1}\right), w^{2}\right) \sigma_{i j} \gamma_{i} \gamma_{j}^{-1} .
$$

To simplify the notation, we will write $\omega_{\gamma}^{x}=\omega(\gamma(x), x), \omega_{\gamma}^{y}=\omega(\gamma(y), y), \omega_{\gamma}^{x, y}=$ $\omega(\gamma(x), y)$.

It is possible to filter the algebra $\mathrm{H}_{t, \mathbf{c}}\left(\Gamma_{l}\right)$ by giving degree 1 to the generators $x_{i}, y_{i}, 1 \leq i \leq l$, and degree 0 to the elements of $\Gamma_{l}$. This filtration will be denoted $F_{\bullet}\left(\mathrm{H}_{t, \mathbf{c}}\left(\Gamma_{l}\right)\right)$ and the corresponding associated graded ring $\operatorname{gr}\left(\mathrm{H}_{t, \mathbf{c}}\left(\Gamma_{l}\right)\right)$.

Theorem 2.1 (PBW Property, EtGi]). The canonical map $\mathrm{H}_{t=0, \mathbf{c}=\mathbf{0}}\left(\Gamma_{l}\right) \longrightarrow$ $\operatorname{gr}\left(\mathrm{H}_{t, \mathbf{c}}\left(\Gamma_{l}\right)\right)$ is an isomorphism.

\section{Double Current algebras}

Before defining $\Gamma$-deformed double current algebras in section [5, we need to prove a series of lemmas for the Lie algebra $\widehat{\mathfrak{s l}}_{n}(\mathbb{C}[u, v] \rtimes \Gamma)$, the universal central extension of $\mathfrak{s l}_{n}(\mathbb{C}[u, v] \rtimes \Gamma)$. In this section, we treat the case $\Gamma=\{\operatorname{id}\}$. We 
will need to assume that $n \geq 4$ in this section and, a fortiori, for the rest of the paper. The first lemma is similar to proposition 3.5 in [MRY] and should admit an analog for other semisimple Lie algebras; however, we doubt that Lemma 3.2 admits such a generalization, except perhaps by adding a few relations. We start with a theorem which gives a description of $\widehat{\mathfrak{s l}}_{n}[u, v]$. We will denote by $\Omega^{1}\left(\mathbb{C}^{2}\right)$ the space of polynomial 1 -forms on the affine plane $\mathbb{C}^{2}$ and by $d(\mathbb{C}[u, v])$ the space of exact 1 -forms on $\mathbb{C}^{2}$.

Theorem 3.1 ([Ka]). The Lie algebra $\widehat{\mathfrak{s l}}_{n}[u, v]$ is isomorphic to the Lie algebra $\mathfrak{s l}_{n}[u, v] \oplus \frac{\Omega^{1}\left(\mathbb{C}^{2}\right)}{d(\mathbb{C}[u, v])}$ with the following bracket (where $(\cdot, \cdot)$ is the Killing form on $\left.\mathfrak{s l}_{n}\right)$ :

$\left[m_{1} \otimes p_{1}, m_{2} \otimes p_{2}\right]=\left[m_{1}, m_{2}\right] \otimes p_{1} p_{2}+\left(m_{1}, m_{2}\right) p_{1} d p_{2}, \quad \forall m_{1}, m_{2} \in \mathfrak{s l}_{n}, p_{1}, p_{2} \in \mathbb{C}[u, v]$ and the elements of $\frac{\Omega^{1}\left(\mathbb{C}^{2}\right)}{d(\mathbb{C}[u, v])}$ are central. $\widehat{A}_{n-1}$ :

We denote by $\widehat{C}_{n-1}=\left(c_{i j}\right)_{0 \leq i, j \leq n-1}$ the $n \times n$ Cartan matrix of affine type

$$
\widehat{C}_{n-1}=\left(\begin{array}{ccccccc}
2 & -1 & 0 & \ldots & \ldots & 0 & -1 \\
-1 & 2 & -1 & 0 & \ldots & \ldots & 0 \\
0 & -1 & 2 & -1 & 0 & \ldots & 0 \\
\vdots & \vdots & \vdots & \vdots & & & \\
0 & \cdots & 0 & -1 & 2 & -1 & 0 \\
0 & \ldots & \ldots & 0 & -1 & 2 & -1 \\
-1 & 0 & \ldots & \ldots & 0 & -1 & 2
\end{array}\right) .
$$

The set of roots of $\mathfrak{s l}_{n}$ will be denoted by $\Delta=\left\{\alpha_{i j} \mid 1 \leq i \neq j \leq n\right\}$ with the choice of positive roots denoted by $\Delta^{+}=\left\{\alpha_{i j} \mid 1 \leq i<j \leq n\right\}$. The longest positive root $\theta$ equals $\alpha_{1 n}$. The elementary matrices will be written $E_{i j}, H_{i}=E_{i i}-E_{i+1, i+1}$ for $1 \leq i \leq n-1$ and $H_{i j}=E_{i i}-E_{j j}$. We set $E_{i}^{+}=E_{i, i+1}, E_{i}^{-}=E_{i+1, i}, 1 \leq i \leq n-1$.

The following is lemma 2.5 in Gu2.

Lemma 3.1. The Lie algebra $\widehat{\mathfrak{s l}}_{n}[u, v]$ is isomorphic to the Lie algebra $\mathfrak{l}$ which is generated by the elements $X_{i, r}^{ \pm}, H_{i, r}, X_{0, r}^{+}, 1 \leq i \leq n-1, r \geq 0$ which satisfy the following relations:

$$
\begin{aligned}
{\left[H_{i, r}, H_{j, s}\right] } & =0 \forall 1 \leq i, j \leq n-1, r, s \geq 0, \\
{\left[H_{i, 0}, X_{j, r}^{ \pm}\right] } & = \pm c_{i j} X_{j, r}^{ \pm} \forall 1 \leq i \leq n-1,0 \leq j \leq n-1, r \geq 0, \\
{\left[X_{i, r}^{ \pm}, X_{i, s}^{ \pm}\right] } & =0,\left[X_{i, r+1}^{ \pm}, X_{j, s}^{ \pm}\right]=\left[X_{i, r}^{ \pm}, X_{j, s+1}^{ \pm}\right], \\
{\left[H_{i, r+1}, X_{j, s}^{ \pm}\right] } & =\left[H_{i, r}, X_{j, s+1}^{ \pm}\right] \forall 1 \leq i, j \leq n-1, r, s \geq 0, \\
{\left[X_{0, r}^{+}, X_{0, s}^{+}\right] } & =0,\left[X_{i, r+1}^{+}, X_{0, s}^{+}\right]=\left[X_{i, r}^{+}, X_{0, s+1}^{+}\right], \\
{\left[H_{i, r+1}, X_{0, s}^{+}\right] } & =\left[H_{i, r}, X_{0, s+1}^{+}\right] \forall 1 \leq i \leq n-1, r, s \geq 0, \\
{\left[X_{i, r}^{+}, X_{j, s}^{-}\right] } & =\delta_{i j} H_{i, r+s}, \forall 0 \leq i \leq n-1,1 \leq j \leq n-1, r, s \geq 0, \\
{\left[X_{i, 0}^{ \pm},\left[X_{i, 0}^{ \pm}, X_{j, r}^{ \pm}\right]\right] } & =0 \forall r \geq 0 \text { if } c_{i j}=-1,\left[X_{i, 0}^{ \pm}, X_{j, r}^{ \pm}\right]=0 \forall r \geq 0 \text { if } c_{i j}=0 .
\end{aligned}
$$

Remark 3.1. In (8), when $i=0$ or $j=0$, we have defining relations only in the + case. 
We will need a simpler set of generators and relations for the Lie algebra $\widehat{\mathfrak{s l}}_{n}[u, v]$, whence the importance of the next lemma, which is lemma 2.7 in Gu2, except for a minor difference.

Lemma 3.2. The Lie algebra $\widehat{\mathfrak{s l}}_{n}[u, v]$ is isomorphic to the Lie algebra $\mathfrak{k}$ which is generated by the elements $X_{i, r}^{ \pm}, H_{i, r}, X_{0, r}^{+}, 1 \leq i \leq n-1, r=0,1$ satisfying the following relations:

(9) $\left[H_{i, r}, H_{j, s}\right]=0,1 \leq i, j \leq n-1, r, s=0,1$,

$\left[H_{i, 0}, X_{j, r}^{ \pm}\right]= \pm c_{i j} X_{j, r}^{ \pm}, 1 \leq i \leq n-1,0 \leq j \leq n-1, r=0,1$,

(10) $\left[X_{i, 0}^{ \pm}, X_{i, 1}^{ \pm}\right]=0,\left[X_{i, 1}^{ \pm}, X_{j, 0}^{ \pm}\right]=\left[X_{i, 0}^{ \pm}, X_{j, 1}^{ \pm}\right]$,

$\left[H_{i, 1}, X_{j, 0}^{ \pm}\right]=\left[H_{i, 0}, X_{j, 1}^{ \pm}\right], 1 \leq i, j \leq n-1$,

$\left[X_{0,0}^{+}, X_{0,1}^{+}\right]=0,\left[X_{i, 1}^{+}, X_{0,0}^{+}\right]=\left[X_{i, 0}^{+}, X_{0,1}^{+}\right]$,

$\left[H_{i, 1}, X_{0,0}^{+}\right]=\left[H_{i, 0}, X_{0,1}^{+}\right], 1 \leq i \leq n-1$,

(12) $\left[X_{i, r}^{+}, X_{j, 0}^{-}\right]=\delta_{i j} H_{i, r}=\left[X_{i, 0}^{+}, X_{j, r}^{-}\right], \forall 0 \leq i \leq n-1,1 \leq j \leq n-1, r=0,1$,

$$
\begin{aligned}
& {\left[X_{i, 0}^{ \pm},\left[X_{i, 0}^{ \pm}, X_{j, 0}^{ \pm}\right]\right]=0 \text { if } c_{i j}=-1} \\
& {\left[X_{i, 0}^{ \pm}, X_{j, 0}^{ \pm}\right]=0 \text { if } c_{i j}=0,0 \leq i, j \leq n-1 .}
\end{aligned}
$$

An isomorphism $\mathfrak{k} \stackrel{\sim}{\longrightarrow} \widehat{\mathfrak{s l}}_{n}[u, v]$ is given by

$X_{i, r}^{ \pm} \mapsto E_{i}^{ \pm} \otimes v^{r}, H_{i, r} \mapsto H_{i} \otimes v^{r}$ for $1 \leq i \leq n-1, X_{0, r}^{+} \mapsto E_{n 1} \otimes u v^{r}, r=0,1$.

We will need a corollary of the previous lemma which gives a fourth presentation of $\widehat{\mathfrak{s l}}_{n}[u, v]$. It is an immediate consequence of Lemma 3.2 since we are only eliminating $X_{0,1}^{+}$from all the relations, so we will use the same letter $\mathfrak{k}$.

Lemma 3.3. The Lie algebra $\widehat{\mathfrak{s l}}_{n}[u, v]$ is isomorphic to the Lie algebra $\mathfrak{k}$ which is generated by the elements $X_{i, r}^{ \pm}, H_{i, r}, X_{0,0}^{+}, 1 \leq i \leq n-1, r=0,1$ satisfying the following relations:

$$
\begin{aligned}
& {\left[H_{i, r}, H_{j, s}\right]=0,1 \leq i, j \leq n-1, r, s=0 \text { or } 1,} \\
& {\left[H_{i, 0}, X_{j, r}^{ \pm}\right]= \pm c_{i j} X_{j, r}^{ \pm}, 1 \leq i, j \leq n-1, r=0,1,} \\
& {\left[X_{i, 0}^{ \pm}, X_{i, 1}^{ \pm}\right]=0,\left[X_{i, 1}^{ \pm}, X_{j, 0}^{ \pm}\right]=\left[X_{i, 0}^{ \pm}, X_{j, 1}^{ \pm}\right],} \\
& {\left[H_{i, 1}, X_{j, 0}^{ \pm}\right]=\left[H_{i, 0}, X_{j, 1}^{ \pm}\right], \forall 1 \leq i, j \leq n-1,} \\
& {\left[X_{j, 1}^{+}, X_{0,0}^{+}\right]=\left[H_{j, 1},\left[X_{j, 0}^{+}, X_{0,0}^{+}\right]\right],} \\
& {\left[H_{1,1}, X_{0,0}^{+}\right]=\left[H_{n-1,1}, X_{0,0}^{+}\right], \forall 1 \leq i, j \leq n-1,} \\
& {\left[X_{0,0}^{+},\left[H_{n-1,1}, X_{0,0}^{+}\right]\right]=0} \\
& {\left[H_{i, 0}, X_{0,0}^{+}\right]=c_{i 0} X_{0,0}^{+},\left[H_{i, 1}, X_{0,0}^{+}\right]=0 \text { if } i \neq 1, n-1,} \\
& {\left[X_{i, r}^{+}, X_{j, s}^{-}\right]=\delta_{i j} H_{i, r+s},\left[\left[H_{n-1, r}, X_{0,0}^{+}\right], X_{j, s}^{-}\right]=0,} \\
& \quad \forall 0 \leq i \leq n-1,1 \leq j \leq n-1,0 \leq r+s \leq 1 \quad(r=0 \text { if } i=0) \\
& {\left[X_{i, 0}^{ \pm},\left[X_{i, 0}^{ \pm}, X_{j, 0}^{ \pm}\right]\right]=0 \text { if } c_{i j}=-1,} \\
& {\left[X_{i, 0}^{ \pm}, X_{j, 0}^{ \pm}\right]=0 \text { if } c_{i j}=0,0 \leq i, j \leq n-1 .}
\end{aligned}
$$

In the previous lemmas, the elements $X_{i, r}^{ \pm}, H_{i, r}$ with $i \neq 0$ generate a Lie subalgebra which is isomorphic to $\mathfrak{s l}_{n}[v]$, whereas those with $r=0$ along with $X_{0,0}^{+}$ 
generate an isomorphic image of $\mathfrak{s l}_{n}[u]$. We would like to end this section by giving one last definition of $\widehat{\mathfrak{s l}}_{n}[u, v]$ in which these two algebras play a more symmetric role, but before that we need to introduce one more lemma (which is probably known to other people).

Lemma 3.4. The Lie algebra $\mathfrak{s l}_{n}[v]$ is isomorphic to the Lie algebra $\mathfrak{f}$ generated by elements $\mathrm{E}_{a b} \in \mathfrak{s l}_{n}, \mathrm{E}_{a b}(v)$ for $1 \leq a \neq b \leq n$ which satisfy the following relations:

$$
\begin{aligned}
{\left[\mathrm{E}_{a b}, \mathrm{E}_{b c}(v)\right] } & =\mathrm{E}_{a c}(v)=\left[\mathrm{E}_{a d}(v), \mathrm{E}_{d c}\right], \\
{\left[\mathrm{E}_{a b}(v), \mathrm{E}_{b c}(v)\right] } & =\left[\mathrm{E}_{a d}(v), \mathrm{E}_{d c}(v)\right] \text { if } a \neq b \neq c \neq a \neq d \neq c . \\
{\left[\mathrm{E}_{a b}, \mathrm{E}_{c d}(v)\right] } & =0=\left[\mathrm{E}_{a b}(v), \mathrm{E}_{c d}(v)\right] \text { if } a \neq b \neq c \neq d \neq a .
\end{aligned}
$$

Proof. We want to define elements $\mathrm{E}_{a b}\left(v^{k}\right) \forall k \geq 2,1 \leq a \neq b \leq n$, by setting inductively $\mathrm{E}_{a b}\left(v^{k+1}\right)=\left[\mathrm{E}_{a c}(v), \mathrm{E}_{c b}\left(v^{k}\right)\right]$ for some $c \neq a, b$. This does not depend on the choice of $c$, for if $d \neq a, b, c$ and (21) is satisfied for $k$ instead of $k+1$, then:

$$
\begin{aligned}
{\left[\mathrm{E}_{a d}(v), \mathrm{E}_{d b}\left(v^{k}\right)\right] } & =\left[\mathrm{E}_{a d}(v),\left[\mathrm{E}_{d c}, \mathrm{E}_{c b}\left(v^{k}\right)\right]\right] \\
& =\left[\left[\mathrm{E}_{a d}(v), \mathrm{E}_{d c}\right], \mathrm{E}_{c b}\left(v^{k}\right)\right]=\left[\mathrm{E}_{a c}(v), \mathrm{E}_{c b}\left(v^{k}\right)\right] .
\end{aligned}
$$

We have to show that

$$
\left[\mathrm{E}_{a b}\left(v^{i}\right), \mathrm{E}_{b c}\left(v^{j}\right)\right]=\mathrm{E}_{a c}\left(v^{i+j}\right) \text { if } i+j=k+1, a \neq b \neq c \neq a
$$

and

$$
\begin{aligned}
{\left[\mathrm{E}_{a b}\left(v^{i}\right), \mathrm{E}_{c d}\left(v^{j}\right)\right]=0 \text { if } i+j } & =k+1 \\
& \text { or } i=1, j=k+1, \text { when } a \neq b \neq c \neq d \neq a .
\end{aligned}
$$

We proceed by induction on $k$, the case $k=0$ being true by the definition of $\mathfrak{f}$. Assume that $i+j=k+1$. Suppose that $a \neq b \neq c \neq a$ and choose $d \neq a, b, c$. First, suppose that $i \geq 1$.

$$
\left[\mathrm{E}_{a b}\left(v^{i}\right), \mathrm{E}_{b c}\left(v^{j}\right)\right]=\left[\left[\mathrm{E}_{a d}(v), \mathrm{E}_{d b}\left(v^{i-1}\right)\right], \mathrm{E}_{b c}\left(v^{j}\right)\right]=\left[\mathrm{E}_{a d}(v), \mathrm{E}_{d c}\left(v^{i+j-1}\right)\right]=\mathrm{E}_{a c}\left(v^{i+j}\right) .
$$

If $i=0, j=k+1$, then

$$
\left[\mathrm{E}_{a b}, \mathrm{E}_{b c}\left(v^{k+1}\right)\right]=\left[\mathrm{E}_{a b},\left[\mathrm{E}_{b d}(v), \mathrm{E}_{d c}\left(v^{k}\right)\right]\right]=\left[\mathrm{E}_{a d}(v), \mathrm{E}_{d c}\left(v^{k}\right)\right]=\mathrm{E}_{a c}\left(v^{k+1}\right) .
$$

We have established (20), so let us turn to (21). If $a \neq b, c \neq a$, choose $d \neq a, b, c$. Then, if $i+j=k+1$ and, without loss of generality, $j \geq 2$,

$$
\left[\mathrm{E}_{a b}\left(v^{i}\right), \mathrm{E}_{a c}\left(v^{j}\right)\right]=\left[\mathrm{E}_{a b}\left(v^{i}\right),\left[\mathrm{E}_{a d}(v), \mathrm{E}_{d c}\left(v^{j-1}\right)\right]\right]=0
$$

by induction. Similarly, [ $\left.\mathrm{E}_{a b}\left(v^{i}\right), \mathrm{E}_{c b}\left(v^{j}\right)\right]=0$ if $i+j=k+1$, and, if $i=1, j=k+1$, we can show that $\left[\mathrm{E}_{a b}(v), \mathrm{E}_{a c}\left(v^{k+1}\right)\right]=0=\left[\mathrm{E}_{a b}(v), \mathrm{E}_{c b}\left(v^{k+1}\right)\right]$.

If $a, b, c, d$ are all distinct and $i+j=k+1,1 \leq i, j \leq k$, then

$$
\begin{aligned}
{\left[\mathrm{E}_{a b}\left(v^{i}\right), \mathrm{E}_{c d}\left(v^{j}\right)\right] } & =\left[\mathrm{E}_{a b}\left(v^{i}\right),\left[\mathrm{E}_{c b}(v), \mathrm{E}_{b d}\left(v^{j-1}\right)\right]\right]=\left[\mathrm{E}_{c b}(v), \mathrm{E}_{a d}\left(v^{i+j-1}\right)\right] \\
& =\left[\mathrm{E}_{c b}(v),\left[\mathrm{E}_{a c}\left(v^{i-1}\right), \mathrm{E}_{c d}\left(v^{j}\right)\right]\right]=-\left[\mathrm{E}_{a b}\left(v^{i}\right), \mathrm{E}_{c d}\left(v^{j}\right)\right] .
\end{aligned}
$$

Comparing the first and last terms, we see that $\left[\mathrm{E}_{a b}\left(v^{i}\right), \mathrm{E}_{c d}\left(v^{j}\right)\right]=0$. If $i=0, j=$ $k+1$, then

$$
\left[\mathrm{E}_{a b}, \mathrm{E}_{c d}\left(v^{k+1}\right)\right]=\left[\mathrm{E}_{a b},\left[\mathrm{E}_{c b}(v), \mathrm{E}_{b d}\left(v^{k}\right)\right]\right]=\left[\mathrm{E}_{c b}(v), \mathrm{E}_{a d}\left(v^{k}\right)\right]=0
$$

by the previous case. The same argument works if $i=k+1, j=0$. 
Finally, if again $a, b, c, d$ are all distinct, we have

$$
\begin{aligned}
{\left[\mathrm{E}_{a b}(v), \mathrm{E}_{c d}\left(v^{k+1}\right)\right] } & =\left[\mathrm{E}_{a b}(v),\left[\mathrm{E}_{c a}\left(v^{k}\right), \mathrm{E}_{a d}(v)\right]\right]=-\left[\mathrm{E}_{c b}\left(v^{k+1}\right), \mathrm{E}_{a d}(v)\right] \\
& \left.=-\left[\left[\mathrm{E}_{c d}\left(v^{k+1}\right), \mathrm{E}_{d b}\right], \mathrm{E}_{a d}(v)\right]\right]=\left[\mathrm{E}_{c d}\left(v^{k+1}\right), \mathrm{E}_{a b}(v)\right] .
\end{aligned}
$$

Comparing the first and last terms shows that $\left[\mathrm{E}_{a b}(v), \mathrm{E}_{c d}\left(v^{k+1}\right)\right]=0$.

Lemma 3.5. The Lie algebra $\widehat{\mathfrak{s l}}_{n}[u, v]$ is isomorphic to the Lie algebra $\mathfrak{t}$ that is generated by the elements $\mathrm{E}_{a b} \in \mathfrak{s l}_{n}, \mathrm{E}_{a b}(u), \mathrm{E}_{a b}(v)$ for $1 \leq a \neq b \leq n$ with the following relations: For any $w_{1}=a_{1} u+b_{1} v, w_{2}=a_{2} u+b_{2} v, a_{i}, b_{i} \in \mathbb{C}$,

$$
\begin{gathered}
{\left[\mathrm{E}_{a b}, \mathrm{E}_{b c}\left(w_{1}\right)\right]=\mathrm{E}_{a c}\left(w_{1}\right)=\left[\mathrm{E}_{a b}\left(w_{1}\right), \mathrm{E}_{b c}\right],} \\
{\left[\mathrm{E}_{a b}\left(w_{1}\right), \mathrm{E}_{b c}\left(w_{2}\right)\right]=\left[\mathrm{E}_{a d}\left(w_{1}\right), \mathrm{E}_{d c}\left(w_{2}\right)\right],} \\
{\left[\mathrm{E}_{a b}\left(w_{1}\right), \mathrm{E}_{b c}\left(w_{2}\right)\right]=\left[\mathrm{E}_{a b}\left(w_{2}\right), \mathrm{E}_{b c}\left(w_{1}\right)\right] \text { if } a \neq b \neq c \neq a \neq d \neq c,} \\
{\left[\mathrm{E}_{a b}\left(w_{1}\right), \mathrm{E}_{c d}\left(w_{2}\right)\right]=0=\left[\mathrm{E}_{a b}, \mathrm{E}_{c d}\left(w_{1}\right)\right] \text { if } a \neq b \neq c \neq d \neq a .}
\end{gathered}
$$

Proof. We can define an epimorphism $\mathfrak{k} \longrightarrow \mathfrak{t}$ by the formulas

$$
\begin{array}{r}
X_{i, 1}^{+} \mapsto \mathrm{E}_{i, i+1}(v), X_{i, 1}^{-} \mapsto \mathrm{E}_{i+1, i}(v), H_{i, 1} \mapsto \mathrm{H}_{i, i+1}(v) \text { for } 1 \leq i \leq n-1, \\
X_{0,0}^{+} \mapsto \mathrm{E}_{n 1}(u) .
\end{array}
$$

We have to check that this respects the relations (14)-(19). We will explain why this is indeed the case for the first equation in (14), the first and second one in (16), the first one in (17) and the second one in (18), but before we do this, we need to deduce a few consequences of the relations in this lemma.

For $a \neq b$, we define $\mathrm{H}_{a b}(w)$ by $\mathrm{H}_{a b}(w)=\left[\mathrm{E}_{a b}(w), \mathrm{E}_{b a}\right]$. Choose $c \neq a, b$, so

$$
\begin{aligned}
\mathrm{H}_{a b}(w) & =\left[\left[\mathrm{E}_{a c}, \mathrm{E}_{c b}(w)\right], \mathrm{E}_{b a}\right]=-\left[\mathrm{E}_{b c}, \mathrm{E}_{c b}(w)\right]+\left[\mathrm{E}_{a c}, \mathrm{E}_{c a}(w)\right] \\
& =-\left[\mathrm{E}_{b c}, \mathrm{E}_{c b}(w)\right]+\left[\left[\mathrm{E}_{a b}, \mathrm{E}_{b c}\right], \mathrm{E}_{c a}(w)\right]=\left[\mathrm{E}_{a b}, \mathrm{E}_{b a}(w)\right]=-\mathrm{H}_{b a}(w) .
\end{aligned}
$$

Starting from $\left[\mathrm{E}_{a b}\left(w_{1}\right), \mathrm{E}_{b c}\left(w_{2}\right)\right]=\left[\mathrm{E}_{a d}\left(w_{1}\right), \mathrm{E}_{d c}\left(w_{2}\right)\right]$ with $a, b, c, d$ all distinct and applying $\left[\cdot, \mathrm{E}_{b a}\right]$ gives the relation

$$
\left[\mathrm{H}_{a b}\left(w_{1}\right), \mathrm{E}_{b c}\left(w_{2}\right)\right]=-\left[\mathrm{E}_{b d}\left(w_{1}\right), \mathrm{E}_{d c}\left(w_{2}\right)\right] .
$$

Although we needed to assume that $a, b, c, d$ were distinct to deduce this equality, it is also true that $\left[\mathrm{H}_{a b}\left(w_{1}\right), \mathrm{E}_{b c}\left(w_{2}\right)\right]=-\left[\mathrm{E}_{b a}\left(w_{1}\right), \mathrm{E}_{a c}\left(w_{2}\right)\right]$ if $a, b, c$ are all distinct, due to relation (23).

Similarly, $\left[\mathrm{H}_{a b}(w), \mathrm{E}_{b c}(w)\right]=-\left[\mathrm{E}_{b d}(w), \mathrm{E}_{d c}(w)\right]$ and commuting both sides with $\mathrm{E}_{c a}$ yields

$$
\left[\mathrm{H}_{a b}(w), \mathrm{E}_{b a}(w)\right]=\left[\mathrm{E}_{c a}(w), \mathrm{E}_{b c}(w)\right]-\left[\mathrm{E}_{b d}(w), \mathrm{E}_{d a}(w)\right]=-2\left[\mathrm{E}_{b d}(w), \mathrm{E}_{d a}(w)\right] .
$$

We now apply $\left[\mathrm{E}_{a b}, \cdot\right]$ to both sides of this equation to get

$$
\left[\mathrm{E}_{a b}(w), \mathrm{E}_{b a}(w)\right]+\left[\mathrm{E}_{b d}(w), \mathrm{E}_{d b}(w)\right]+\left[\mathrm{E}_{d a}(w), \mathrm{E}_{a d}(w)\right] .
$$

This is a useful equation since it helps us deduce the following for $a, b, c$ all distinct:

$$
\begin{aligned}
{\left[\mathrm{H}_{a b}(w), \mathrm{H}_{b c}(w)\right] } & =\left[\left[\mathrm{E}_{a b}(w), \mathrm{E}_{b a}\right],\left[\mathrm{E}_{b c}, \mathrm{E}_{c b}(w)\right]\right] \\
& =\left[\left[\mathrm{E}_{a c}(w), \mathrm{E}_{b a}\right], \mathrm{E}_{c b}(w)\right]-\left[\mathrm{E}_{b c},\left[\mathrm{E}_{a b}(w), \mathrm{E}_{c a}(w)\right]\right] \\
& =-\left[\mathrm{E}_{b c}(w), \mathrm{E}_{c b}(w)\right]+\left[\mathrm{E}_{a c}(w), \mathrm{E}_{c a}(w)\right]-\left[\mathrm{E}_{a b}(w), \mathrm{E}_{b a}(w)\right]=0 .
\end{aligned}
$$


The first equation in (14) is now an immediate consequence of (27). Applying $\left[\mathrm{E}_{12}, \cdot\right]$ to $0=\left[\mathrm{E}_{n-1,1}(u), \mathrm{E}_{21}(v)\right]$ gives $0=-\left[\mathrm{E}_{n-1,2}(u), \mathrm{E}_{21}(v)\right]+\left[\mathrm{E}_{n-1,1}(u), \mathrm{H}_{12}(v)\right]$. Therefore,

$$
\begin{aligned}
-\left[\mathrm{H}_{12}(v), \mathrm{E}_{n-1,1}(u)\right] & =\left[\mathrm{E}_{n-1,2}(u), \mathrm{E}_{21}(v)\right] \\
& =\left[\mathrm{E}_{n-1, n}(v), \mathrm{E}_{n 1}(u)\right]=\left[\mathrm{H}_{n-1, n}(v), \mathrm{E}_{n-1,1}(u)\right],
\end{aligned}
$$

the last equality being a consequence of applying $\left[\cdot, \mathrm{E}_{n, n-1}\right]$ to $0=\left[\mathrm{E}_{n-1, n}(v)\right.$, $\left.\mathrm{E}_{n-1,1}(u)\right]$. We now use $\left[\mathrm{E}_{n, n-1}, \cdot\right]$ again to obtain

$$
\begin{aligned}
& -\left[\mathrm{H}_{12}(v), \mathrm{E}_{n 1}(u)\right]=\left[\mathrm{H}_{n-1, n}(v), \mathrm{E}_{n 1}(u)\right]+2\left[\mathrm{E}_{n, n-1}(v), \mathrm{E}_{n-1,1}(u)\right] \\
& \quad=\left[\mathrm{H}_{n-1, n}(v), \mathrm{E}_{n 1}(u)\right]+2\left[\mathrm{H}_{n, n-1}(v), \mathrm{E}_{n 1}(u)\right]=-\left[\mathrm{H}_{n-1, n}(v), \mathrm{E}_{n 1}(u)\right] .
\end{aligned}
$$

This implies that the second relation in (16) is respected. As for the first relation in (16) when $j=n-1$ or $j=1$, it is a consequence of (25) with $w_{1}=v, w_{2}=u$.

The first relation in (17) and the second one in (18) follow also from (25) and from (24).

To prove that it is an isomorphism, we would like to construct an inverse $\xi$. We do this by using Lemma 3.3, which identifies $\mathfrak{k}$ with the Lie algebra in Theorem 3.1 , We set $\xi\left(\mathrm{E}_{a b}(u)\right)=E_{a b} \otimes u$ and $\xi\left(\mathrm{E}_{a b}(v)\right)=E_{a b} \otimes v$. Clearly, this defines a Lie algebra map $\mathfrak{t} \rightarrow \widehat{\mathfrak{s l}}_{n}[u, v]$. Taking the composite with the map $\mathfrak{k} \rightarrow \mathfrak{t}$ above yields a homomorphism $\mathfrak{k} \rightarrow \widehat{\mathfrak{s l}}_{n}[u, v]$ which is the isomorphism given in Lemma 3.3 (see the formulas after Lemma 3.2). Therefore, $\mathfrak{k} \stackrel{\sim}{\longrightarrow} \mathfrak{t}$.

\section{The universal Central extension of $\mathfrak{s l}_{n}(\mathbb{C}[u, v] \rtimes \Gamma)$}

For an arbitrary associative algebra $A, \mathfrak{s l}_{n}(A)$ is defined as the space of matrices in $\mathfrak{g l}_{n}(A)$ with trace in $[A, A]$. This Lie algebra is perfect, so it admits a universal central extension whose kernel is isomorphic to the first cyclic homology group $\mathrm{HC}_{1}(A)$ KaLo. When $A$ is the group ring $A=\mathbb{C}[\Gamma], \mathrm{HC}_{1}(A)=0$ (see chapter 9 in $[\mathrm{We}]$, so we conclude that $\mathfrak{s l}_{n}(\mathbb{C}[\Gamma])$ is universally closed. Therefore, Theorem 4.1 gives a description of $\mathfrak{s l}_{n}(\mathbb{C}[\Gamma])$. We will need to use the following theorem of C. Kassel and J.L. Loday in the case $A=\mathbb{C}[u, v] \rtimes \Gamma$. We will compute later in this section $\mathrm{HC}_{1}(\mathbb{C}[u, v] \rtimes \Gamma)$.

Theorem 4.1 ([KaLo $)$. Let $A$ be an associative algebra over $\mathbb{C}$. The universal central extension $\widehat{\mathfrak{s l}}_{n}(A)$ of $\mathfrak{s l}_{n}(A)$ is the Lie algebra generated by elements $F_{a b}(p), 1 \leq a \neq b \leq n, p \in A$, satisfying the following relations:

$$
\begin{gathered}
F_{a b}\left(t_{1} p_{1}+t_{2} p_{2}\right)=t_{1} F_{a b}\left(p_{1}\right)+t_{2} F_{a b}\left(p_{2}\right) \quad t_{1}, t_{2} \in \mathbb{C}, p_{1}, p_{2} \in A, \\
{\left[F_{a b}\left(p_{1}\right), F_{b c}\left(p_{2}\right)\right]=F_{a c}\left(p_{1} p_{2}\right) \text { if } a \neq b \neq c \neq a,} \\
{\left[F_{a b}\left(p_{1}\right), F_{c d}\left(p_{2}\right)\right]=0 \text { if } a \neq b \neq c \neq d \neq a .}
\end{gathered}
$$

We will need to simplify Theorem 4.1] when $A=\mathbb{C}[u, v] \rtimes \Gamma$. A generalization of the following lemma, under the extra condition that $n \geq 5$, is given by proposition 3.3 in $\mathrm{Gu} 3$.

Lemma 4.1. The universal central extension $\widehat{\mathfrak{s l}}_{n}(\mathbb{C}[u, v] \rtimes \Gamma)$ is isomorphic to the Lie algebra e generated by elements $\mathrm{E}_{a b}(w), w=t u+s v, \mathrm{E}_{a b}(\gamma), 1 \leq a \neq b \leq n, \gamma \in$ $\Gamma, s, t \in \mathbb{C}$ such that the following relations hold: If $a \neq b \neq c \neq a \neq d \neq c$ and $w_{i}=t_{i} u+s_{i} v, i=1,2$ :

$$
\mathrm{E}_{a b}(w)=t \mathrm{E}_{a b}(u)+s \mathrm{E}_{a b}(v), \quad\left[\mathrm{E}_{a b}\left(w_{1}\right), \mathrm{E}_{b c}\left(w_{2}\right)\right]=\left[\mathrm{E}_{a d}\left(w_{2}\right), \mathrm{E}_{d c}\left(w_{1}\right)\right],
$$




$$
\left[\mathrm{E}_{a b}(\gamma), \mathrm{E}_{b c}(w)\right]=\left[\mathrm{E}_{a d}(\gamma(w)), \mathrm{E}_{d c}(\gamma)\right], \quad\left[\mathrm{E}_{a b}\left(\gamma_{1}\right), \mathrm{E}_{b c}\left(\gamma_{2}\right)\right]=\mathrm{E}_{a c}\left(\gamma_{1} \gamma_{2}\right) .
$$

If $a \neq b \neq c \neq d \neq a$ :

$$
\left[\mathrm{E}_{a b}\left(w_{1}\right), \mathrm{E}_{c d}\left(w_{2}\right)\right]=0=\left[\mathrm{E}_{a b}\left(\gamma_{1}\right), \mathrm{E}_{c d}\left(\gamma_{2}\right)\right]=\left[\mathrm{E}_{a b}(\gamma), \mathrm{E}_{c d}(w)\right] .
$$

Proof. We will introduce elements $\mathrm{E}_{a b}(q)$ for any $q \in \mathbb{C}[u, v] \rtimes \Gamma$ and show that they satisfy the relations in Theorem 4.1, When $q \in \mathbb{C}[u, v]$, the elements $\mathrm{E}_{a b}(q)$ can be defined using the map $\widehat{\mathfrak{s l}}_{n}[u, v] \longrightarrow \mathfrak{e}$ given by Lemma 3.5 and Theorem 4.1 in the case $A=\mathbb{C}[u, v]$. Suppose $q=p \gamma, p \in \mathbb{C}[u, v], \gamma \in \Gamma$. We can assume that $p=u^{e_{1}} v^{e_{2}}$. Set $e=e_{1}+e_{2}$; we will use induction on $e$. Choose $a \neq b$ and $c \neq a, b$; set $\mathrm{E}_{a b}(q)=\left[\mathrm{E}_{a c}(p), \mathrm{E}_{c b}(\gamma)\right]$. We claim that this definition of $\mathrm{E}_{a b}(q)$ does not depend on the choice of $c$. (This is true when the degree of $p$ is one according to (32).) Indeed, suppose that $d \neq a, b, c$ and $e_{1}, e_{2} \geq 1$ (the cases $e_{1}=0$ or $e_{2}=0$ are similar) and write $\mathrm{E}_{a c}(p)=\left[\mathrm{E}_{a d}(v), \mathrm{E}_{d c}\left(u^{e_{1}} v^{e_{2}-1}\right)\right]$. Arguing by induction, we can assume that $\left[\mathrm{E}_{d b}\left(u^{e_{1}} v^{e_{2}-1}\right), \mathrm{E}_{d b}(\gamma)\right]=0$. Then

$$
\begin{aligned}
\mathrm{E}_{a b}(q) & =\left[\left[\mathrm{E}_{a d}(v), \mathrm{E}_{d c}\left(u^{e_{1}} v^{e_{2}-1}\right)\right], \mathrm{E}_{c b}(\gamma)\right]=\left[\mathrm{E}_{a d}(v),\left[\mathrm{E}_{d c}\left(u^{e_{1}} v^{e_{2}-1}\right), \mathrm{E}_{c b}(\gamma)\right]\right] \\
& =\left[\mathrm{E}_{a d}(v),\left[\mathrm{E}_{d c}\left(u^{e_{1}} v^{e_{2}-1}\right),\left[\mathrm{E}_{c d}, \mathrm{E}_{d b}(\gamma)\right]\right]\right] \\
& =\left[\mathrm{E}_{a d}(v),\left[\left[\mathrm{E}_{d c}\left(u^{e_{1}} v^{e_{2}-1}\right), \mathrm{E}_{c d}\right], \mathrm{E}_{d b}(\gamma)\right]\right] \\
& =\left[\mathrm{E}_{a d}\left(u^{e_{1}} v^{e_{2}}\right), \mathrm{E}_{d b}(\gamma)\right]+\left[\left[\mathrm{E}_{d c}\left(u^{e_{1}} v^{e_{2}-1}\right), \mathrm{E}_{c d}\right], \mathrm{E}_{a b}(v \gamma)\right] \\
& =\left[\mathrm{E}_{a d}\left(u^{e_{1}} v^{e_{2}}\right), \mathrm{E}_{d b}(\gamma)\right]
\end{aligned}
$$

since $\left[\mathrm{E}_{d c}\left(u^{e_{1}} v^{e_{2}-1}\right), \mathrm{E}_{a b}(v \gamma)\right]=\frac{1}{2}\left[\left[\mathrm{H}_{d c}(u), \mathrm{E}_{d c}\left(u^{e_{1}-1} v^{e_{2}-1}\right)\right], \mathrm{E}_{a b}(v \gamma)\right]=0$ by induction.

Let us assume that $\left[\mathrm{E}_{a b}\left(p_{1} \gamma_{1}\right), \mathrm{E}_{b c}\left(p_{2} \gamma_{2}\right)\right]=\mathrm{E}_{a c}\left(p_{1} \gamma_{1}\left(p_{2}\right) \gamma_{1} \gamma_{2}\right)$ for any $a \neq b \neq$ $c \neq a$ and also that $\left[\mathrm{E}_{a b}\left(p_{1} \gamma_{1}\right), \mathrm{E}_{c d}\left(p_{2} \gamma_{2}\right)\right]=0$ for any $a \neq b \neq c \neq d \neq a$, any $\gamma_{1}, \gamma_{2} \in \Gamma$ and any $p_{1}, p_{2} \in \mathbb{C}[u, v]$ of total degree $<e$. We want to prove that the same relations hold when the total degree of $p_{1}, p_{2}$ is $e$.

Step 1: Suppose that $a, b, c, d$ are all distinct and $e_{1} \geq 1$. Set $\widetilde{p}=u^{e_{1}-1} v^{e_{2}}$, so $p=u \widetilde{p}$. Using induction, we get

$$
\begin{aligned}
{\left[\mathrm{E}_{a b}(\gamma), \mathrm{E}_{b c}(p)\right] } & =\left[\mathrm{E}_{a b}(\gamma),\left[\mathrm{E}_{b d}(u), \mathrm{E}_{d c}(\widetilde{p})\right]\right]=\left[\left[\mathrm{E}_{a b}(\gamma), \mathrm{E}_{b d}(u)\right], \mathrm{E}_{d c}(\widetilde{p})\right] \\
& =\left[\left[\mathrm{E}_{a b}(\gamma(u)), \mathrm{E}_{b d}(\gamma)\right], \mathrm{E}_{d c}(\widetilde{p})\right] \\
& =\left[\mathrm{E}_{a b}(\gamma(u)),\left[\mathrm{E}_{b d}(\gamma), \mathrm{E}_{d c}(\widetilde{p})\right]\right]=\left[\mathrm{E}_{a b}(\gamma(u)), \mathrm{E}_{b c}(\gamma(\widetilde{p}) \gamma)\right] \\
& =\left[\mathrm{E}_{a b}(\gamma(u)),\left[\mathrm{E}_{b d}(\gamma(\widetilde{p})), \mathrm{E}_{d c}(\gamma)\right]\right]=\left[\left[\mathrm{E}_{a b}(\gamma(u)), \mathrm{E}_{b d}(\gamma(\widetilde{p}))\right], \mathrm{E}_{d c}(\gamma)\right] \\
& =\left[\mathrm{E}_{a d}(\gamma(u \widetilde{p})), \mathrm{E}_{d c}(\gamma)\right]=\mathrm{E}_{a c}(\gamma(p) \gamma) .
\end{aligned}
$$

Step 2: Assume that $a \neq b \neq c \neq d \neq a$ and $e_{1} \geq 1$. There are three subcases to consider: $a, b, c, d$ are all distinct, $a=c, b=d$. In the first subcase, $\left[\mathrm{E}_{a b}(p), \mathrm{E}_{c d}(\gamma)\right]=\frac{1}{2}\left[\left[\mathrm{H}_{a b}(u), \mathrm{E}_{a b}(\widetilde{p})\right], \mathrm{E}_{c d}(\gamma)\right]=0$ by induction. In the second subcase, choose $e \neq a, c, d$; then $\left[\mathrm{E}_{a b}(p), \mathrm{E}_{a d}(\gamma)\right]=\left[\left[\mathrm{E}_{a e}(u), \mathrm{E}_{e b}(\widetilde{p})\right], \mathrm{E}_{a d}(\gamma)\right]=0$ by induction since $\operatorname{deg}(\widetilde{p})<e$. The third subcase is similar to the second one.

Step 3: Choose $a, b, c, d$ all distinct. We know from step 2 that $\left[\mathrm{E}_{a b}\left(p_{1}\right), \mathrm{E}_{d c}\left(\gamma_{2}\right)\right]$ $=0$, so

$$
\begin{aligned}
{\left[\mathrm{E}_{a b}\left(p_{1}\right), \mathrm{E}_{b c}\left(p_{2} \gamma_{2}\right)\right] } & =\left[\mathrm{E}_{a b}\left(p_{1}\right),\left[\mathrm{E}_{b d}\left(p_{2}\right), \mathrm{E}_{d c}\left(\gamma_{2}\right)\right]\right]=\left[\left[\mathrm{E}_{a b}\left(p_{1}\right), \mathrm{E}_{b d}\left(p_{2}\right)\right], \mathrm{E}_{d c}\left(\gamma_{2}\right)\right] \\
& =\left[\mathrm{E}_{a d}\left(p_{1} p_{2}\right), \mathrm{E}_{d c}\left(\gamma_{2}\right)\right]=\mathrm{E}_{a c}\left(p_{1} p_{2} \gamma_{2}\right) .
\end{aligned}
$$


Step 4: Again, suppose that $a, b, c, d$ are all distinct. Then

$$
\begin{aligned}
{\left[\mathrm{E}_{a b}\left(p_{1} \gamma_{1}\right), \mathrm{E}_{b c}\left(p_{2}\right)\right] } & =\left[\left[\mathrm{E}_{a d}\left(p_{1}\right), \mathrm{E}_{d b}\left(\gamma_{1}\right)\right], \mathrm{E}_{b c}\left(p_{2}\right)\right]=\left[\mathrm{E}_{a d}\left(p_{1}\right),\left[\mathrm{E}_{d b}\left(\gamma_{1}\right), \mathrm{E}_{b c}\left(p_{2}\right)\right]\right] \\
& =\left[\mathrm{E}_{a d}\left(p_{1}\right), \mathrm{E}_{d c}\left(\gamma_{1}\left(p_{2}\right) \gamma_{1}\right)\right]=\mathrm{E}_{a c}\left(p_{1} \gamma_{1}\left(p_{2}\right) \gamma_{1}\right) .
\end{aligned}
$$

The last equality is a consequence of step 3.

Step 5: Assume that $a \neq b \neq c \neq d \neq a$. As in step 2, there are three subcases to consider. In the first subcase, using step 2 twice, we get

$$
\left[\mathrm{E}_{a b}\left(p_{1} \gamma_{1}\right), \mathrm{E}_{c d}\left(\gamma_{2}\right)\right]=\left[\left[\mathrm{E}_{a d}\left(p_{1}\right), \mathrm{E}_{d b}\left(\gamma_{1}\right)\right], \mathrm{E}_{c d}\left(\gamma_{2}\right)\right]=-\left[\mathrm{E}_{a d}\left(p_{1}\right), \mathrm{E}_{c b}\left(\gamma_{2} \gamma_{1}\right)\right]=0
$$

In the second subcase, choosing $e \neq a, b, d$, we get $\left[\mathrm{E}_{a b}\left(p_{1} \gamma_{1}\right), \mathrm{E}_{a c}\left(\gamma_{2}\right)\right]=\left[\left[\mathrm{E}_{a e}\left(p_{1}\right)\right.\right.$, $\left.\left.\mathrm{E}_{e b}\left(\gamma_{1}\right)\right], \mathrm{E}_{a d}\left(\gamma_{2}\right)\right]=0$. The third subcase is similar to the second one.

Step 6: Suppose that $a, b, c, d$ are all distinct. Then

$$
\begin{aligned}
{\left[\mathrm{E}_{a b}\left(p_{1} \gamma_{1}\right), \mathrm{E}_{b c}\left(p_{2} \gamma_{2}\right)\right] } & =\left[\mathrm{E}_{a b}\left(p_{1} \gamma_{1}\right),\left[\mathrm{E}_{b d}\left(p_{2}\right), \mathrm{E}_{d c}\left(\gamma_{2}\right)\right]\right] \\
& =\left[\left[\mathrm{E}_{a b}\left(p_{1} \gamma_{1}\right), \mathrm{E}_{b d}\left(p_{2}\right)\right], \mathrm{E}_{d c}\left(\gamma_{2}\right)\right] \\
& =\left[\mathrm{E}_{a d}\left(p_{1} \gamma_{1}\left(p_{2}\right) \gamma_{1}\right), \mathrm{E}_{d c}\left(\gamma_{2}\right)\right] \\
& =\left[\left[\mathrm{E}_{a b}\left(p_{1} \gamma_{1}\left(p_{2}\right)\right), \mathrm{E}_{b d}\left(\gamma_{1}\right)\right], \mathrm{E}_{d c}\left(\gamma_{2}\right)\right] \\
& =\left[\mathrm{E}_{a b}\left(p_{1} \gamma_{1}\left(p_{2}\right)\right),\left[\mathrm{E}_{b d}\left(\gamma_{1}\right), \mathrm{E}_{d c}\left(\gamma_{2}\right)\right]\right] \\
& =\left[\mathrm{E}_{a b}\left(p_{1} \gamma_{1}\left(p_{2}\right)\right), \mathrm{E}_{b c}\left(\gamma_{1} \gamma_{2}\right)\right] \\
& =\mathrm{E}_{a c}\left(p_{1} \gamma_{1}\left(p_{2}\right) \gamma_{1} \gamma_{2}\right) .
\end{aligned}
$$

Step 7: Finally, suppose that $a \neq b \neq c \neq d \neq a$ and $q_{1}=p_{1} \gamma_{1}, q_{2}=p_{2} \gamma_{2}$. As in steps 2 and 5, there are three subcases. In the first case,

$$
\begin{aligned}
{\left[\mathrm{E}_{a b}\left(q_{1}\right), \mathrm{E}_{c d}\left(q_{2}\right)\right] } & =\left[\left[\mathrm{E}_{a c}\left(q_{1}\right), \mathrm{E}_{c b}\right], \mathrm{E}_{c d}\left(q_{2}\right)\right]=\left[\mathrm{E}_{a d}\left(q_{1} q_{2}\right), \mathrm{E}_{c b}\right] \\
& =\left[\left[\mathrm{E}_{a b}\left(q_{1}\right), \mathrm{E}_{b d}\left(q_{2}\right)\right], \mathrm{E}_{c b}\right]=-\left[\mathrm{E}_{a b}\left(q_{1}\right), \mathrm{E}_{c d}\left(q_{2}\right)\right] .
\end{aligned}
$$

Comparing the first and last terms, we conclude that $\left[\mathrm{E}_{a b}\left(q_{1}\right), \mathrm{E}_{c d}\left(q_{2}\right)\right]=0$. In the second case, suppose that $a, b, d$ are all distinct and choose $e \neq a, b, d$. Then $\left[\mathrm{E}_{a b}\left(q_{1}\right), \mathrm{E}_{a d}\left(q_{2}\right)\right]=\left[\left[\mathrm{E}_{a e}, \mathrm{E}_{e b}\left(q_{1}\right)\right], \mathrm{E}_{a d}\left(q_{2}\right)\right]=0$ by the previous subcase and step 2. The third case can be handled as the second one.

As recalled earlier, the center of $\widehat{\mathfrak{s l}}_{n}(\mathbb{C}[u, v] \rtimes \Gamma)$ is known to be isomorphic to $\mathrm{HC}_{1}(\mathbb{C}[u, v] \rtimes \Gamma)$; see $[\mathrm{KaLO}$. Consequently, the following proposition will be useful:

Proposition 4.1. The first cyclic homology group of $\mathbb{C}[u, v] \rtimes \Gamma$ is isomorphic to $\Omega^{1}(\mathbb{C}[u, v])^{\Gamma} / d\left(\mathbb{C}[u, v]^{\Gamma}\right)$, the quotient of the space of $\Gamma$-invariant 1 -forms on the complex affine plane by the space of exact forms coming from $\Gamma$-invariant polynomials.

Proof. It is proved in $[\mathrm{Fa}]$ that the Hochschild homology of $\mathbb{C}[u, v] \rtimes \Gamma$ is given by:

$$
\begin{gathered}
\mathrm{HH}_{0}(\mathbb{C}[u, v] \rtimes \Gamma)=\mathbb{C}[u, v]^{\Gamma} \oplus \mathbb{C}^{c l(\Gamma)-1}, \\
\mathrm{HH}_{1}(\mathbb{C}[u, v] \rtimes \Gamma)=\left(\mathbb{C}[u, v] \otimes_{\mathbb{C}} U\right)^{\Gamma} \text { where } U \cong \operatorname{span}\{u, v\} \cong \mathbb{C}^{2}, \\
\mathrm{HH}_{2}(\mathbb{C}[u, v] \rtimes \Gamma) \cong \mathbb{C}[u, v]^{\Gamma}, \quad \mathrm{HH}_{i}(\mathbb{C}[u, v] \rtimes \Gamma)=0 \text { for } i \geq 3 .
\end{gathered}
$$

Here, $\operatorname{cl}(\Gamma)$ is the number of conjugacy classes of $\Gamma$. There exists an exact sequence $\mathrm{HH}_{0}(\mathbb{C}[u, v] \rtimes \Gamma) \longrightarrow \mathrm{HH}_{1}(\mathbb{C}[u, v] \rtimes \Gamma) \longrightarrow \mathrm{HC}_{1}(\mathbb{C}[u, v] \rtimes \Gamma) \longrightarrow 0$ and the first map is given by the differential $d$, the space $\mathbb{C}[u, v] \otimes U$ being identified with the space of regular 1 -forms on $\mathbb{C}^{2} \cong U$. 
Proposition 4.2. The first cyclic homology group of $\mathbb{C}[u, v] \rtimes \Gamma$ can be identified as a vector space with $\mathbb{C}[u, v]^{\Gamma}$.

Proof. The form $\omega$ (see section 2 ) allows us to identify $\Omega^{1}(\mathbb{C}[u, v])$ with the space $\mathcal{V}$ of polynomial vector fields on $\mathbb{C}^{2}$. (We assume here that $\omega(u, v)=1$.) There is a contraction map $\mathcal{V} \rightarrow \mathbb{C}[u, v]$, so we can define a linear map $\Omega^{1}(\mathbb{C}[u, v]) \longrightarrow \mathbb{C}[u, v]$ given explicitly by $u^{s} v^{r} d u \mapsto r u^{s} v^{r-1}, u^{s} v^{r} d v \mapsto-s u^{s-1} v^{r}$. Since $\omega$ is $\Gamma$-invariant, this restricts to a surjective map $\Omega^{1}(\mathbb{C}[u, v])^{\Gamma} \longrightarrow \mathbb{C}[u, v]^{\Gamma}$ and the kernel of this last map is $d(\mathbb{C}[u, v])^{\Gamma}$, which equals $d\left(\mathbb{C}[u, v]^{\Gamma}\right)$. Thus, $\mathrm{HC}_{1}(\mathbb{C}[u, v] \rtimes \Gamma) \cong \mathbb{C}[u, v]^{\Gamma}$.

These two propositions suggest that it may be possible to relate the enveloping algebra of $\widehat{\mathfrak{s l}}_{n}(\mathbb{C}[u, v] \rtimes \Gamma)$ to $\mathfrak{U g l}_{n}\left(A_{1} \rtimes \Gamma\right)$, where $A_{1}$ is the first Weyl algebra: this is explained in section 7

In the last section, we will consider deformations of an algebra related to $\widehat{U}_{\mathfrak{s} l}(\mathbb{C}[u, v] \rtimes \Gamma) ;$ namely, $\mathfrak{U}_{\mathfrak{s l}}\left(\mathbb{C}\left[u^{ \pm 1}, w\right] \rtimes \Gamma\right)$ when $\Gamma \cong \mathbb{Z} / d \mathbb{Z}$ is cyclic acts trivially on $w$ and on $u$ by $\xi(u)=\zeta u, \xi$ being a generator of $\mathbb{Z} / d \mathbb{Z}$ and $\zeta$ a primitive $d^{\text {th }}$-root of unity. It is explained in [GHL that $\mathfrak{s l}_{n}\left(\mathbb{C}\left[u^{ \pm 1}, w\right] \rtimes \Gamma\right) \cong \mathfrak{s l}_{n d}\left(\mathbb{C}\left[s^{ \pm 1}, w\right]\right)$ : this follows from the isomorphism of associative algebras given in loc. cit. $\mathbb{C}\left[u^{ \pm 1}\right] \rtimes \Gamma \cong$ $M_{d}\left(\mathbb{C}\left[s^{ \pm 1}\right]\right)$, where $s=u^{d}$. It follows that $\operatorname{HC}_{0}\left(\mathbb{C}\left[u^{ \pm 1}, w\right] \rtimes \Gamma\right) \cong \mathbb{C}\left[s^{ \pm 1}, w\right] \cong$ $\mathbb{C}\left[u^{ \pm 1}, w\right]^{\Gamma}$ and

$$
\mathrm{HC}_{1}\left(\mathbb{C}\left[u^{ \pm 1}, w\right] \rtimes \Gamma\right) \cong \frac{\Omega^{1}\left(\mathbb{C}\left[s^{ \pm 1}, w\right]\right)}{d\left(\mathbb{C}\left[s^{ \pm 1}, w\right]\right)} \cong \mathbb{C} \cdot s^{-1} d s \oplus \mathbb{C}\left[s^{ \pm 1}, w\right] w d s .
$$

Let $\mathfrak{g}$ be the Lie algebra defined by the relations in Definition 9.5 when $\lambda=$ $0, \beta=\mathbf{0}$. The next proposition will be useful to understand the algebras in section 9 .

Proposition 4.3. The Lie algebra $\mathfrak{g}$ is isomorphic to $\widehat{\mathfrak{s l}}_{n}\left(\mathbb{C}\left[u^{ \pm 1}, w\right] \rtimes \Gamma\right)$.

Proof. See proposition 4.4 in [GHL and also [MRY]. An isomorphism is given by

$$
\begin{gathered}
\text { For } 1 \leq i \leq n-1, \quad X_{i, r, \mathbf{j}}^{ \pm 1} \mapsto E_{i}^{ \pm} \otimes w^{r} \mathbf{e}_{j}, H_{i, r, \mathbf{j}} \mapsto H_{i} \otimes w^{r} \mathbf{e}_{j}, \\
X_{0, r, \mathbf{j}}^{+} \mapsto E_{n 1} \otimes w^{r} u \mathbf{e}_{j}, X_{0, r, \mathbf{j}}^{-} \mapsto E_{1 n} \otimes w^{r} u^{-1} \mathbf{e}_{j}, \\
H_{0, r, \mathbf{j}} \mapsto E_{n n} \otimes w^{r} \mathbf{e}_{j}-E_{11} \otimes w^{r} \mathbf{e}_{j+1}+\delta_{0 \mathbf{j}} s^{-1} w^{r} d s .
\end{gathered}
$$

Here, we identify the center of $\mathfrak{s l}_{n}\left(\mathbb{C}\left[u^{ \pm 1}, w\right] \rtimes \Gamma\right.$ with $\mathrm{HC}_{1}\left(\mathbb{C}\left[u^{ \pm 1}, w\right] \rtimes \Gamma\right)$ as above.

Let $\mathfrak{a}$ be the Lie subalgebra of $\mathfrak{g}$ generated by $X_{i, r, \mathbf{j}}^{ \pm}, H_{i, r, \mathbf{j}}, X_{0, r, \mathbf{j}}^{+}, X_{0, r+1, \mathbf{j}}^{-}$for $1 \leq i \leq n-1, r \geq 0,0 \leq j \leq d-1$. Via the isomorphism $\mathfrak{g} \stackrel{\sim}{\longrightarrow} \widehat{\mathfrak{s l}}_{n}\left(\mathbb{C}\left[u^{ \pm 1}, w\right] \rtimes \Gamma\right)$, we see that $\mathfrak{a}$ contains $\widehat{\mathfrak{s l}}_{n}(\mathbb{C}[u, v] \rtimes \Gamma)$ with $v=u^{-1} w$. As we have mentioned earlier, we are interested in deformations of the enveloping algebra of a Lie algebra slightly bigger than $\widehat{\mathfrak{s l}}_{n}(\mathbb{C}[u, v] \rtimes \Gamma)$. The projection $\mathfrak{g} \rightarrow \mathfrak{g} /\left\langle\sum_{i=0}^{n-1} \sum_{j=0}^{d-1} H_{i, 0, \mathbf{j}}\right\rangle=\widetilde{\mathfrak{g}}$ is injective on $\mathfrak{a}$ (note that $\sum_{i=0}^{n-1} \sum_{j=0}^{d-1} H_{i, 0, \mathbf{j}}$ is a central element of $\mathfrak{g}$ ), so we can view $\widehat{\mathfrak{s l}}_{n}(\mathbb{C}[u, v] \rtimes \Gamma)$ as contained in the Lie subalgebra $\widetilde{\mathfrak{a}}$ of $\widetilde{\mathfrak{g}}$ which is the image of $\mathfrak{a}$ under the previous projection. The Lie subalgebra of $\widetilde{\mathfrak{g}}$ generated by $\widetilde{X}_{i, 0, \mathbf{j}}^{ \pm}, \widetilde{H}_{i, 0, \mathbf{j}}, \widetilde{H}_{0,0, \mathbf{j}}, \widetilde{X}_{0,0, \mathbf{j}}^{+}$for $1 \leq i \leq n-1,0 \leq j \leq d-1$ is larger than $\mathfrak{s l}_{n}(\mathbb{C}[u] \rtimes \Gamma)$. (Here, ${ }^{\sim}$ denotes the image under the projection $\mathfrak{g} \rightarrow \widetilde{\mathfrak{g}}$.) For instance, $\sum_{i=0}^{n-1} \widetilde{H}_{i, 0, \mathbf{j}}=$ $E_{11}\left(\mathbf{e}_{j}-\mathbf{e}_{j+1}\right) \notin \mathfrak{s l}_{n}(\mathbb{C}[u] \rtimes \Gamma)$. (Note that $\operatorname{span}\left\{\mathbf{e}_{j}-\mathbf{e}_{j+1} \mid 0 \leq j \leq d-1\right\}=$ 
$\bigoplus_{j=1}^{d-1} \mathbb{C} \cdot \xi^{j} \subsetneq \mathbb{C}[\Gamma]$.) In the last section, we will explain how $\Gamma$-DDCA in the cyclotomic case are deformations of $\mathfrak{U} \mathfrak{\mathfrak { a }}$.

\section{5. $\Gamma$-DEFORMED DOUBLE CURRENT ALGEBRAS}

We introduce in this section a new family of quantum algebras which are deformations of the enveloping algebra of an enlargement of $\widehat{\mathfrak{s l}}_{n}(\mathbb{C}[u, v] \rtimes \Gamma)$ and which are related to symplectic reflection algebras for wreath products of $\Gamma$ via a functor of Schur-Weyl type. (By enlargement of a Lie algebra $\mathfrak{g}$, we mean a Lie algebra $\check{\mathfrak{g}}$ which contains a.) Before defining them, we need to introduce the Lie algebra $\mathfrak{g l}_{n}(\mathbb{C}[\Gamma])$ which is the Lie subalgebra of $\mathfrak{g l}_{n}(\mathbb{C}[\Gamma])$ spanned by $\mathfrak{s l}_{n}(\mathbb{C}[\Gamma])$ and by the elements $E_{a a}(\gamma) \forall \gamma \in \Gamma \backslash\{\mathrm{id}\}, 1 \leq a \leq n$. The necessity to consider $\widetilde{\mathfrak{g l}}_{n}(\mathbb{C}[\Gamma])$ instead of just $\mathfrak{s l}_{n}(\mathbb{C}[\Gamma])$ will become clear in sections 6 and 7 .

We will use the following notation: given an algebra $A$ and elements $a_{1}, a_{2} \in A$, we will set $S\left(a_{1}, a_{2}\right)=a_{1} a_{2}+a_{2} a_{1}$.

Definition 5.1. The $\Gamma$-deformed double current algebra $D_{\beta, \mathbf{b}}^{n}$ with parameters $\beta \in \mathbb{C}, \mathbf{b} \in \mathbf{Z} \Gamma, \mathbf{b}=\lambda \cdot \mathrm{id}+\sum_{\gamma \in \Gamma \backslash\{\mathrm{id}\}} b_{\gamma} \gamma$ is the algebra generated by the elements of $\widetilde{\mathfrak{g l}}_{n}(\mathbb{C}[\Gamma]), \mathrm{E}_{a b}\left(t_{1} w_{1}+t_{2} w_{2}\right)$ for $1 \leq a \neq b \leq n, t_{1}, t_{2} \in \mathbb{C}, w, w_{1}, w_{2} \in U$ which satisfy $\mathrm{E}_{a b}\left(t_{1} w_{1}+t_{2} w_{2}\right)=t_{1} \mathrm{E}_{a b}\left(w_{1}\right)+t_{2} \mathrm{E}_{a b}\left(w_{2}\right)$ and the following relations: If $a \neq b \neq c \neq a \neq d \neq c$,

$$
\begin{aligned}
& {\left[\mathrm{E}_{a b}(\gamma), \mathrm{E}_{b c}(w)\right] }=\left[\mathrm{E}_{a d}(\gamma(w)), \mathrm{E}_{d c}(\gamma)\right], \\
& {\left[\mathrm{E}_{a a}(\gamma), \mathrm{E}_{a c}(w)\right] }=\left[\mathrm{E}_{a b}(\gamma), \mathrm{E}_{b c}(w)\right]=\left[\mathrm{E}_{a c}(\gamma(w)), \mathrm{E}_{c c}(\gamma)\right], \\
& {\left[\mathrm{E}_{a b}\left(w_{2}\right), \mathrm{E}_{b c}\left(w_{1}\right)\right]=\left[\mathrm{E}_{a d}\left(w_{1}\right), \mathrm{E}_{d c}\left(w_{2}\right)\right]+\omega\left(w_{1}, w_{2}\right) \mathrm{E}_{a c}(\mathbf{b}+\beta) } \\
&+\frac{\lambda}{8} \omega\left(w_{1}, w_{2}\right) \sum_{\gamma \in \Gamma} \sum_{i, j=1}^{n}( S\left(\left[\mathrm{E}_{a b}\left(\gamma^{-1}\right), \mathrm{E}_{i j}\right],\left[\mathrm{E}_{j i}, \mathrm{E}_{b c}(\gamma)\right]\right) \\
&+\left.S\left(\left[\mathrm{E}_{a d}(\gamma), \mathrm{E}_{i j}\right],\left[\mathrm{E}_{j i}, \mathrm{E}_{d c}\left(\gamma^{-1}\right)\right]\right)\right) \\
&-\frac{\lambda}{2} \sum_{\gamma \in \Gamma}\left(\omega\left(\gamma\left(w_{1}\right), w_{2}\right)-\omega\left(w_{1}, w_{2}\right)\right)\left(\mathrm{E}_{b b}\left(\gamma^{-1}\right) \mathrm{E}_{a c}(\gamma)+\mathrm{E}_{d d}(\gamma) \mathrm{E}_{a c}\left(\gamma^{-1}\right)\right) .
\end{aligned}
$$

If $a, b, c$ are all distinct, then $\left[\mathrm{E}_{c c}(\gamma), \mathrm{E}_{a b}(w)\right]=0$, and if $a \neq b \neq c \neq d \neq a$, then $\left[\mathrm{E}_{a b}(\gamma), \mathrm{E}_{c d}(w)\right]=0$ and

$$
\left[\mathrm{E}_{a b}\left(w_{1}\right), \mathrm{E}_{c d}\left(w_{2}\right)\right]=\frac{\lambda}{4} \sum_{\gamma \in \Gamma} \omega\left(\gamma\left(w_{1}\right), w_{2}\right) S\left(\mathrm{E}_{a d}\left(\gamma^{-1}\right), \mathrm{E}_{c b}(\gamma)\right) .
$$

Set $\widetilde{\mathbf{b}}=\sum_{\gamma \in \Gamma \backslash\{\mathrm{id}\}} b_{\gamma} \gamma$, so $\widetilde{\mathbf{b}}=\mathbf{b}(\lambda=0)$. Let $\widetilde{\mathrm{D}}_{\beta, \widetilde{\mathbf{b}}}^{n}$ be the subalgebra of $\mathrm{D}_{\beta, \mathbf{b}(\lambda=0)}^{n}$ generated by the elements $\mathrm{E}_{a b}\left(w_{1}\right), \mathrm{E}_{a b}\left(w_{2}\right), \mathrm{E}_{a b}(\gamma)$ for $a \neq b$. Lemma 4.1 says that $\widetilde{\mathrm{D}}_{\beta=0, \mathbf{b}=0}^{n}$ is isomorphic to the enveloping algebra of $\widehat{\mathfrak{s l}}_{n}(\mathbb{C}[u, v] \rtimes \Gamma)$. When $\beta=1, \lambda=0=b_{\gamma}$ for $\gamma \neq \mathrm{id}, \mathrm{D}_{\beta=1, \mathbf{b}=\mathbf{0}}^{n}$ is exactly the enveloping algebra of $\mathfrak{g l}_{n}\left(A_{1} \rtimes \Gamma\right)$, where $A_{1}$ is the first Weyl algebra. $\mathrm{D}_{\beta=0, \mathbf{b}=\mathbf{0}}^{n}$ is the enveloping algebra of a Lie algebra that we denote $\check{\mathfrak{s l}}_{n}(\mathbb{C}[u, v] \rtimes \Gamma)$ and we have $\check{\mathfrak{s l}}_{n}(\mathbb{C}[u, v] \rtimes \Gamma) \supset$ $\widehat{\mathfrak{s l}}_{n}(\mathbb{C}[u, v] \rtimes \Gamma)$. See section 7 for more details.

When $\Gamma$ is the trivial group, $\mathrm{D}_{\beta, \mathbf{b}}^{n}$ is isomorphic to the algebra $\mathrm{D}_{\lambda, \beta}^{n}$ in $\mathrm{Gu} 2$. (see also Gu1]). The main difference in the definitions of $\mathrm{D}_{\beta, \mathbf{b}}^{n}$ and $\mathrm{D}_{\lambda, \beta}^{n}$ is that the former does not involve any Yangian. Actually, there does not seem to be any 
sensible notion of Yangian associated to $\Gamma$ in general, which, from a heuristic point of view, is not surprising since, when $\Gamma$ is not cyclic, $\mathbb{C}^{2}$ is not the direct sum of two one-dimensional $\Gamma$-invariant subspaces. When $\Gamma$ is a finite cyclic group, we can give another definition of $\mathrm{D}_{\beta, \mathbf{b}}^{n}$ which involves Yangians: see section 9 .

By giving degree 0 to the elements of $\tilde{\mathfrak{g l}}_{n}(\mathbb{C}[\Gamma])$ and degree one to $\mathrm{E}_{a b}(w), w \in U$, we can define a filtration $F_{\bullet}$ on $\mathrm{D}_{\beta, \mathbf{b}}^{n}$ such that $\mathrm{D}_{\beta=0, \mathbf{b}=\mathbf{0}}^{n} \rightarrow \operatorname{gr}_{F}\left(\mathrm{D}_{\beta, \mathbf{b}}^{n}\right)$. The PBW theorem in section 8 says that this canonical map is an isomorphism.

\section{Schur-Weyl FunCtor AND EQUivalence of CATEGORIES}

Given a right module $M$ over $\mathrm{H}_{t, \mathbf{c}}\left(\Gamma_{l}\right)$, we set $\mathrm{SW}(M)=M \otimes_{\mathbb{C}\left[S_{l}\right]}\left(\mathbb{C}^{n}\right)^{\otimes l}$. We would like to give $\mathrm{SW}(M)$ a structure of a left module over $\mathrm{D}_{\beta, \mathbf{b}}^{n}$. The action of $\mathrm{E}_{a b}$ is simply via the $\mathfrak{s l}_{n}$-module structure on $\left(\mathbb{C}^{n}\right)^{\otimes l}$. Let us assume that $u, v, x, y \in U$ are such that the map $u \mapsto x, v \mapsto y$ is a $\Gamma$-equivariant automorphism of the symplectic vector space $U$, so that $\omega(\gamma(u), v)=\omega(\gamma(x), y)$. In particular, $\{u, v\}$ is a symplectic basis of $\mathbb{C}^{2}$. We would like to let $\mathrm{E}_{a b}(w), \mathrm{E}_{a b}(\gamma) \in \mathrm{D}_{\lambda, \beta}^{n}$ act on $\operatorname{SW}(M)$ in the following way:

$$
\mathrm{E}_{a b}(w)(m \otimes \mathbf{v})=\sum_{k=1}^{l} m w_{k} \otimes E_{a b}^{(k)}(\mathbf{v}), \quad \mathrm{E}_{a b}(\gamma)(m \otimes \mathbf{v})=\sum_{k=1}^{l} m \gamma_{k}^{-1} \otimes E_{a b}^{(k)}(\mathbf{v})
$$

Here, $w_{k}=t_{1} x_{k}+t_{2} y_{k}$ if $w=t_{1} x+t_{2} y, \mathbf{v}=v_{i_{1}} \otimes \cdots \otimes v_{i_{l}} \in \mathbb{C}^{n}$ and

$$
E_{a b}^{(k)}(\mathbf{v})=v_{i_{1}} \otimes \cdots \otimes v_{i_{k-1}} \otimes E_{a b}\left(v_{i_{k}}\right) \otimes v_{i_{k+1}} \otimes \cdots \otimes v_{i_{l}}
$$

These operators define a representation of $\mathrm{D}_{\beta, \mathbf{b}}^{n}$ on $\mathrm{SW}(M)$ if and only if the following relations hold between $t, \mathbf{c}, \lambda, \mathbf{b}: \lambda=\kappa, b_{\gamma}=c_{\gamma^{-1}}$ for $\gamma \neq \mathrm{id}$ and $\beta=t-\frac{n \kappa|\Gamma|}{4}-\kappa$.

To prove our claim, we have to verify that the operators above satisfy the defining relations of $\mathrm{D}_{\beta, \mathbf{b}}^{n}$. We start by computing that, for $a \neq b \neq c \neq a \neq d \neq c$,

$$
\begin{aligned}
& \left(\left[\mathrm{E}_{a b}(u), \mathrm{E}_{b c}(u)\right]-\left[\mathrm{E}_{a d}(u), \mathrm{E}_{d c}(u)\right]\right)(m \otimes \mathbf{v}) \\
& =\sum_{\substack{j, k=1 \\
j \neq k}}^{l} m\left(x_{k} x_{j}-x_{j} x_{k}\right) \otimes\left(E_{a b}^{(j)} E_{b c}^{(k)}-E_{a d}^{(j)} E_{d c}^{(k)}\right)(\mathbf{v}) \\
& =-\frac{\kappa}{2} \sum_{\substack{j, k=1 \\
j \neq k}}^{l} \sum_{\gamma \in \Gamma} \omega_{\gamma}^{x} m \sigma_{k j} \gamma_{k} \gamma_{j}^{-1} \otimes\left(E_{a b}^{(j)} E_{b c}^{(k)}-E_{a d}^{(j)} E_{d c}^{(k)}\right)(\mathbf{v}) \\
& =-\frac{\kappa}{2} \sum_{\substack{j, k=1 \\
j \neq k}}^{l} \sum_{\gamma \in \Gamma} \omega_{\gamma}^{x} m \gamma_{j} \gamma_{k}^{-1} \otimes\left(E_{b b}^{(j)} E_{a c}^{(k)}-E_{d d}^{(j)} E_{a c}^{(k)}\right)(\mathbf{v}) \\
& =-\frac{\kappa}{2} \sum_{\substack{j, k=1 \\
j \neq k}}^{l} \sum_{\gamma \in \Gamma} \omega_{\gamma}^{x} m \gamma_{j} \gamma_{k}^{-1} \otimes H_{b d}^{(j)} E_{a c}^{(k)}(\mathbf{v})=-\frac{\kappa}{2} \sum_{\gamma \in \Gamma} \omega_{\gamma}^{x} \mathrm{H}_{b d}\left(\gamma^{-1}\right) \mathrm{E}_{a c}(\gamma)(m \otimes \mathbf{v}) .
\end{aligned}
$$


The computations are the same when $u$ is replaced by $v$ and $\omega_{\gamma}^{x}$ is replaced by $\omega_{\gamma}^{y}$. Under the same assumption on $a, b, c, d$, we now compute:

$$
\begin{aligned}
& \left(\left[\mathrm{E}_{a b}(v), \mathrm{E}_{b c}(u)\right]-\left[\mathrm{E}_{a d}(u), \mathrm{E}_{d c}(v)\right]\right)(m \otimes \mathbf{v}) \\
& =\sum_{k=1}^{l} m\left(x_{k} y_{k}-y_{k} x_{k}\right) \otimes E_{a c}^{(k)}(\mathbf{v})+\sum_{\substack{j, k=1 \\
j \neq k}}^{l} m\left[x_{k}, y_{j}\right] \otimes E_{a b}^{(j)} E_{b c}^{(k)}(\mathbf{v}) \\
& -\sum_{\substack{j, k=1 \\
j \neq k}}^{l} m\left[y_{j}, x_{k}\right] \otimes E_{a d}^{(k)} E_{d c}^{(j)}(\mathbf{v}) \\
& =\sum_{k=1}^{l} m\left(t+\frac{\kappa}{2} \sum_{\substack{j, k=1 \\
j \neq k}}^{l} \sum_{\gamma \in \Gamma} \gamma_{j} \gamma_{k}^{-1} \sigma_{j k}+\sum_{\gamma \in \Gamma \backslash\{\mathrm{id}\}} c_{\gamma} \gamma_{k}\right) \otimes E_{a c}^{(k)}(\mathbf{v}) \\
& -\frac{\kappa}{2} \sum_{\substack{j, k=1 \\
j \neq k}}^{l} \sum_{\gamma \in \Gamma} \omega_{\gamma}^{x, y} m \gamma_{j} \gamma_{k}^{-1} \sigma_{j k} \otimes E_{a b}^{(j)} E_{b c}^{(k)}(\mathbf{v}) \\
& -\frac{\kappa}{2} \sum_{\substack{j, k=1 \\
j \neq k}}^{l} \sum_{\gamma \in \Gamma} \omega_{\gamma}^{x, y} m \gamma_{j} \gamma_{k}^{-1} \sigma_{j k} \otimes E_{a d}^{(k)} E_{d c}^{(j)}(\mathbf{v}) \\
& =\mathrm{E}_{a c}\left(t+\sum_{\gamma \in \Gamma \backslash\{\mathrm{id}\}} c_{\gamma} \gamma^{-1}\right)(m \otimes \mathbf{v})+\frac{\kappa}{2} \sum_{j \neq k} \sum_{\gamma \in \Gamma} \sum_{e=1}^{n} m \gamma_{j} \gamma_{k}^{-1} \otimes E_{a e}^{(j)} E_{e c}^{(k)}(\mathbf{v}) \\
& -\frac{\kappa}{2} \sum_{\substack{j, k=1 \\
j \neq k}}^{l} \sum_{\gamma \in \Gamma} \omega_{\gamma}^{x, y} m \gamma_{j} \gamma_{k}^{-1} \otimes E_{b b}^{(j)} E_{a c}^{(k)}(\mathbf{v}) \\
& -\frac{\kappa}{2} \sum_{\substack{j, k=1 \\
j \neq k}}^{l} \sum_{\gamma \in \Gamma} \omega_{\gamma}^{x, y} m \gamma_{j} \gamma_{k}^{-1} \otimes E_{d d}^{(k)} E_{a c}^{(j)}(\mathbf{v}) \\
& =\mathrm{E}_{a c}\left(t+\sum_{\gamma \in \Gamma \backslash\{\mathrm{id}\}} c_{\gamma} \gamma^{-1}-\frac{\kappa n}{4}|\Gamma|\right)(m \otimes \mathbf{v}) \\
& +\frac{\kappa}{4} \sum_{\gamma \in \Gamma} \sum_{e=1, e \neq a, c}^{n} S\left(\mathrm{E}_{a e}\left(\gamma^{-1}\right), \mathrm{E}_{e c}(\gamma)\right)(m \otimes \mathbf{v}) \\
& +\frac{\kappa}{8} \sum_{\gamma \in \Gamma}\left(S\left(\mathrm{H}_{a b}\left(\gamma^{-1}\right), \mathrm{E}_{a c}(\gamma)\right)+S\left(\mathrm{H}_{c d}(\gamma), \mathrm{E}_{a c}\left(\gamma^{-1}\right)\right)+S\left(\mathrm{H}_{a d}(\gamma), \mathrm{E}_{a c}\left(\gamma^{-1}\right)\right)\right. \\
& \left.+S\left(\mathrm{H}_{c b}\left(\gamma^{-1}\right), \mathrm{E}_{a c}(\gamma)\right)\right)(m \otimes \mathbf{v}) \\
& -\frac{\kappa}{2} \sum_{\gamma \in \Gamma}\left(\omega_{\gamma}^{x, y}-1\right)\left(\mathrm{E}_{b b}\left(\gamma^{-1}\right) \mathrm{E}_{a c}(\gamma)+\mathrm{E}_{d d}(\gamma) \mathrm{E}_{a c}\left(\gamma^{-1}\right)\right)(m \otimes \mathbf{v}) .
\end{aligned}
$$


We now check that $\left(\left[\mathrm{E}_{a b}(\gamma), \mathrm{E}_{b c}(u)\right]-\left[\mathrm{E}_{a d}(\gamma(u)), \mathrm{E}_{d c}(\gamma)\right]\right)(m \otimes \mathbf{v})$

$$
\begin{aligned}
& =\sum_{k=1}^{l} m\left(x_{k} \gamma_{k}^{-1}-\gamma_{k}^{-1} \gamma_{k}\left(x_{k}\right)\right) \otimes E_{a c}^{(k)}(\mathbf{v})+\sum_{\substack{j, k=1 \\
j \neq k}}^{l} m\left[x_{k}, \gamma_{j}^{-1}\right] \otimes E_{a b}^{(j)} E_{b c}^{(k)}(\mathbf{v}) \\
& -\sum_{\substack{j, k=1 \\
j \neq k}}^{l} m\left[\gamma_{k}^{-1}, \gamma_{j}\left(x_{j}\right)\right] \otimes E_{a d}^{(j)} E_{d c}^{(k)}(\mathbf{v}) \\
& =0
\end{aligned}
$$

since $\gamma_{k}^{-1} \gamma_{k}\left(x_{k}\right)=x_{k} \gamma_{k}^{-1}$ in $\mathrm{H}_{t, \mathbf{c}}\left(\Gamma_{l}\right)$ and $\gamma_{j} x_{k}=x_{k} \gamma_{j}, \forall \gamma \in \Gamma$ if $j \neq k$. Exactly the same computations work with $v$ instead of $u$ and in the case $a=b$ or $c=d$ with $\gamma \neq i d$.

Now, we will assume instead that $a \neq b \neq c \neq d \neq a$ :

$$
\begin{aligned}
{\left[\mathrm{E}_{a b}(u), \mathrm{E}_{c d}(v)\right](m \otimes \mathbf{v}) } & =\sum_{j \neq k} m\left[y_{j}, x_{k}\right] \otimes E_{a b}^{(k)} E_{c d}^{(j)}(\mathbf{v}) \\
& =\frac{\kappa}{2} \sum_{\gamma \in \Gamma} \sum_{j \neq k} \omega_{\gamma}^{x, y} m \sigma_{j k} \gamma_{k} \gamma_{j}^{-1} \otimes E_{a b}^{(k)} E_{c d}^{(j)}(\mathbf{v}) \\
& =\frac{\kappa}{2} \sum_{\gamma \in \Gamma} \sum_{j \neq k} \omega_{\gamma}^{x, y} m \gamma_{j} \gamma_{k}^{-1} \otimes E_{c b}^{(k)} E_{a d}^{(j)}(\mathbf{v}) \\
& =\frac{\kappa}{2} \sum_{\gamma \in \Gamma} \omega_{\gamma}^{x, y} \mathrm{E}_{c b}(\gamma) \mathrm{E}_{a d}\left(\gamma^{-1}\right)(m \otimes \mathbf{v}) .
\end{aligned}
$$

The computations are the same for $\left[\mathrm{E}_{a b}(u), \mathrm{E}_{c d}(u)\right](m \otimes \mathbf{v})$ and $\left[\mathrm{E}_{a b}(v), \mathrm{E}_{c d}(v)\right](m \otimes \mathbf{v})$ with $\omega_{\gamma}^{x}\left(\right.$ resp. $\left.\omega_{\gamma}^{y}\right)$ instead of $\omega_{\gamma}^{x, y}$.

We can state what we have proved so far in this section, but before that we need a definition.

Definition 6.1. A module over $D_{\beta, \mathbf{b}}^{n}$ is called integrable if it is a direct sum of integral weight spaces under the action of $\mathfrak{h}$ and is locally nilpotent under the action of $E_{a b}(w)$ for any $1 \leq a \neq b \leq n$ and any $w \in U$.

Definition 6.2. A module over $\mathrm{D}_{\beta, \mathbf{b}}^{n}$ is said to be of level $l$ if, as a module over $\mathfrak{s l}_{n}$, it decomposes as a direct sum of irreducible $\mathfrak{s l}_{n}$-submodules of $\left(\mathbb{C}^{n}\right)^{\otimes l}$.

Proposition 6.1. Suppose that $\lambda=\kappa, \beta=t-\frac{\kappa n|\Gamma|}{4}-\kappa$ and $b_{\gamma}=c_{\gamma^{-1}}$ for $\gamma \neq \mathrm{id}$. Then there exists a functor $\mathrm{SW}: \bmod _{R}-\mathrm{H}_{t, \mathbf{c}}\left(\Gamma_{l}\right) \longrightarrow \bmod _{L}^{\text {int,l }}-\mathrm{D}_{\beta, \mathbf{b}}^{n}$ given by $\operatorname{SW}(M)=M \otimes_{\mathbb{C}\left[S_{l}\right]}\left(\mathbb{C}^{n}\right)^{\otimes l}$. Here, $\bmod _{L}^{\text {int,l }}$ is the category of integrable left modules of level $l$.

This proposition can be strengthened to yield a new generalization of the classical Schur-Weyl duality theorem between $\mathfrak{s l}_{n}$ and $S_{l}$.

Theorem 6.1. Suppose that $\lambda=\kappa, \beta=t-\frac{\kappa n|\Gamma|}{4}-\kappa$ and $b_{\gamma}=c_{\gamma^{-1}}$ for $\gamma \neq$ id. If $l+2<n$, then the functor SW yields an equivalence between the category of right $\mathrm{H}_{t, \mathbf{c}}\left(\Gamma_{l}\right)$-modules and the category of left modules over $\mathrm{D}_{\beta, \mathbf{b}}^{n}$ which are integrable of level $l$.

The proof of this theorem will follow the same lines as the analogous result in Gu1, Gu2 (see also ChPr1, VaVa1]). However, before proving it, we have to 
establish a similar result for $\Gamma_{l}$ and $\mathfrak{s l}_{n}(\mathbb{C}[\Gamma]$ ). (When $\Gamma$ is the cyclic group $\mathbb{Z} / d \mathbb{Z}$, a more general result was established in ATY] in the context of cyclotomic Hecke algebras and where $\mathfrak{s l}_{n}(\mathbb{C}[\Gamma]) \cong \mathfrak{s l}_{n}^{\oplus d}$ is replaced by $\left.\mathfrak{g l}_{m_{1}} \oplus \cdots \oplus \mathfrak{g l}_{m_{d}}.\right)$ We will need the following lemma.

Lemma 6.1. If $\mathbf{v}=v_{i_{1}} \otimes \cdots \otimes v_{i_{l}}$ is a generator of $\left(\mathbb{C}^{n}\right)^{\otimes l}$ as a module over $\mathfrak{s l}_{n}$ (e.g., if $i_{j} \neq i_{k}$ for any $j \neq k$ ), then $m \otimes \mathbf{v}=0 \Longrightarrow m=0$.

Proposition 6.2. The functor $S W: \bmod _{R}-\mathbb{C}\left[\Gamma_{l}\right] \longrightarrow \bmod _{L}^{l}-\mathfrak{s l}_{n}(\mathbb{C}[\Gamma])$ is given by $M \mapsto M \otimes_{\mathbb{C}\left[S_{l}\right]}\left(\mathbb{C}^{n}\right)^{\otimes l}$, which is an equivalence of categories of finite-dimensional modules when $l+1<n$.

Proof. Given a right $\Gamma_{l}$-module $M$, we can put on $M \otimes_{\mathbb{C}\left[S_{l}\right]}\left(\mathbb{C}^{n}\right)^{\otimes l}$ a structure of a left module over $\mathfrak{s l}_{n}(\mathbb{C}[\Gamma])$ by setting

$$
\mathrm{E}_{a b}(\gamma)(m \otimes \mathbf{v})=\sum_{k=1}^{l} m \gamma_{k}^{-1} \otimes E_{a b}^{(k)}(\mathbf{v}) .
$$

This extends the classical Schur-Weyl functor to $\bmod _{R}-\mathbb{C}\left[\Gamma_{l}\right]$ and $\bmod _{L}^{l}-\mathfrak{s l}_{n}(\mathbb{C}[\Gamma])$. The second part of the proposition requires more work; to prove it, we will follow the approach and ideas in ChPr1.

Suppose that $l+1<n$. Let $N$ be a left $\mathfrak{s l}_{n}(\mathbb{C}[\Gamma])$-module which is of level $l$ as an $\mathfrak{s l}_{n}$-module. Then $N \cong M \otimes_{\mathbb{C}\left[S_{l}\right]}\left(\mathbb{C}^{n}\right)^{\otimes l}$ as a left $\mathfrak{s l}_{n}$-module for some right $S_{l}$-module $M$ by the classical case. We want to show that $M$ is a right module over the group $\Gamma_{l}$.

For $1 \leq k \leq l$, set $\mathbf{v}^{(k)}=v_{2} \otimes \cdots \otimes v_{k} \otimes v_{n} \otimes v_{k+1} \otimes \cdots \otimes v_{l}$, where $\left\{v_{1}, v_{2}, \ldots, v_{n}\right\}$ is the standard basis of $\mathbb{C}^{n}$. Let $\mathbf{w}^{(k)}$ be the same element of $\left(\mathbb{C}^{n}\right)^{\otimes l}$ as $\mathbf{v}^{(k)}$ except that $v_{n}$ is replaced by $v_{1}$. As in [ChPr1], we write $\mathbf{w}_{\tau}^{(k)}$ for the element obtained by permuting the factors of $\mathbf{w}^{(k)}$ by $\tau \in S_{l}$. The set $\left\{\mathbf{w}_{\tau}^{(k)} \mid \tau \in S_{l}\right\}$ is a basis for the subspace of $\left(\mathbb{C}^{n}\right)^{\otimes l}$ of weight $\lambda_{l}=\epsilon_{1}+\cdots+\epsilon_{l}$, where $\epsilon_{i}$ is the weight on diagonal matrices given by $E_{j j} \mapsto \delta_{i j}$, so we can write

$$
\mathrm{E}_{1 n}(\gamma)\left(m \otimes \mathbf{v}^{(k)}\right)=\sum_{\tau \in S_{l}} m_{\tau} \otimes \mathbf{w}_{\tau}^{(k)}
$$

for some $m_{\tau} \in M$. This can be rewritten as $\mathrm{E}_{1 n}(\gamma)\left(m \otimes \mathbf{v}^{(k)}\right)=m^{\prime} \otimes \mathbf{w}^{(k)}$ for some $m^{\prime} \in M$. By Lemma 6.1 above, $m^{\prime}$ is unique, so there exists a linear endomorphism $\zeta_{1 n}^{\gamma, k}$ of $M$ such that $m^{\prime}=\zeta_{1 n}^{\gamma, k}(m)$ for all $m \in M$.

One can show, exactly as in lemma 4.5 in ChPr1, that $\mathrm{E}_{1 n}(\gamma)(m \otimes \mathbf{v})=$ $\sum_{k=1}^{n} \zeta_{1 n}^{\gamma, k}(m) \otimes E_{1 n}^{(k)}(\mathbf{v})$ for any $\mathbf{v} \in\left(\mathbb{C}^{n}\right)^{\otimes l}$. Instead of the quantized Serre relation that they use, one should consider the relation $\left[\mathrm{E}_{n, n-1},\left[\mathrm{E}_{n, n-1}, \mathrm{E}_{1 n}(\gamma)\right]\right]=0$, which is a consequence of $\left[\mathrm{E}_{1 n}(\gamma), \mathrm{E}_{n, n-1}\right]=\left[\mathrm{E}_{1, n-2}(\gamma), \mathrm{E}_{n-2, n-1}\right]$.

Similarly, it is possible to show also that, for any $1 \leq a \neq b \leq n$, there exists an endomorphism $\zeta_{a b}^{\gamma, k} \in \operatorname{End}_{\mathbb{C}}(M)$ such that $\mathrm{E}_{a b}(\gamma)(m \otimes \mathbf{v})=\sum_{k=1}^{l} \zeta_{a b}^{\gamma, k}(m) \otimes E_{a b}^{(k)}(\mathbf{v})$. We claim that, for any choice of $a \neq b, c \neq d, \zeta_{a b}^{\gamma, k}=\zeta_{c d}^{\gamma, k}$. Suppose, for instance, that $b \neq d \neq a$, Then $\mathrm{E}_{a d}(\gamma)=\left[\mathrm{E}_{a b}(\gamma), \mathrm{E}_{b d}\right]$, so

$$
\mathrm{E}_{a d}(\gamma)(m \otimes \mathbf{v})=\left[\mathrm{E}_{a b}(\gamma), \mathrm{E}_{b d}\right](m \otimes \mathbf{v})=\sum_{k=1}^{l} \zeta_{a b}^{\gamma, k}(m) \otimes E_{a d}^{(k)}(\mathbf{v}) .
$$


Since this is true for any $\mathbf{v} \in\left(\mathbb{C}^{n}\right)^{l}, m \in M, \zeta_{a b}^{\gamma, k}=\zeta_{a d}^{\gamma, k}$. The other cases can be treated similarly. Therefore, we can define $\zeta^{\gamma, k} \in \operatorname{End}_{\mathbb{C}}(M)$ unambiguously by setting $\zeta^{\gamma, k}=\zeta_{a b}^{\gamma, k}$ for any choice of $a \neq b$.

We can now show that setting $m \gamma_{k}=\zeta^{\gamma^{-1}, k}(m)$ gives $M$ a structure of a right module over $\Gamma_{l}$. We will prove the following relations:

1. $\left(m \gamma_{k}\right) \tilde{\gamma}_{k}=m(\gamma \tilde{\gamma})_{k}, \forall \gamma, \tilde{\gamma} \in \Gamma$.

2. $\left(m \gamma_{k}\right) \tilde{\gamma}_{j}=\left(m \tilde{\gamma}_{j}\right) \gamma_{k} \forall \gamma, \widetilde{\gamma} \in \Gamma$ if $j \neq k$.

3. $\left(m \sigma_{j k}\right) \gamma_{j}=\left(m \gamma_{k}\right) \sigma_{j k}, \forall 1 \leq j \neq k \leq l, \forall \gamma \in \Gamma$.

(1): Set $\mathbf{v}=v_{2} \otimes \cdots \otimes v_{k} \otimes v_{n} \otimes v_{k+1} \otimes \cdots \otimes v_{l}$ and $\widetilde{\mathbf{v}}=v_{2} \otimes \cdots \otimes v_{k} \otimes v_{1} \otimes$ $v_{k+1} \otimes \cdots \otimes v_{l}$. Since $\left[\mathrm{E}_{1, n-1}\left(\tilde{\gamma}^{-1}\right), \mathrm{E}_{n-1, n}\left(\gamma^{-1}\right)\right]=\mathrm{E}_{1 n}\left(\tilde{\gamma}^{-1} \gamma^{-1}\right)$, we obtain

$$
\left[\mathrm{E}_{1, n-1}\left(\tilde{\gamma}^{-1}\right), \mathrm{E}_{n-1, n}\left(\gamma^{-1}\right)\right](m \otimes \mathbf{v})=\left(\left(m \gamma_{k}\right) \tilde{\gamma}_{k}\right) \otimes \widetilde{\mathbf{v}}=\mathrm{E}_{1 n}\left(\tilde{\gamma}^{-1} \gamma^{-1}\right)(m \otimes \mathbf{v}) .
$$

This equality, along with Lemma 6.1, imply that $\left(m \gamma_{k}\right) \tilde{\gamma}_{k}=m(\gamma \tilde{\gamma})_{k}$, which is what we wanted.

(2): Suppose that $1 \leq j<k \leq l$. Set $\mathbf{v}=v_{3} \otimes \cdots \otimes v_{j+1} \otimes v_{n} \otimes v_{j+2} \otimes \cdots \otimes v_{k} \otimes$ $v_{n-1} \otimes v_{k+1} \otimes \cdots \otimes v_{l}$ and $\widetilde{\mathbf{v}}=v_{3} \otimes \cdots \otimes v_{j+1} \otimes v_{1} \otimes v_{j+2} \otimes \cdots \otimes v_{k} \otimes v_{2} \otimes v_{k+1} \otimes \cdots \otimes v_{l}$. Since $\left[\mathrm{E}_{1 n}\left(\tilde{\gamma}^{-1}\right), \mathrm{E}_{2, n-1}\left(\gamma^{-1}\right)\right]=0$, we get

$$
0=\left[\mathrm{E}_{1 n}\left(\tilde{\gamma}^{-1}\right), \mathrm{E}_{2, n-1}\left(\gamma^{-1}\right)\right](m \otimes \mathbf{v})=\left(\left(m \gamma_{k}\right) \tilde{\gamma}_{j}\right) \otimes \widetilde{\mathbf{v}}-\left(\left(m \tilde{\gamma}_{j}\right) \gamma_{k}\right) \otimes \widetilde{\mathbf{v}},
$$

so, by Lemma 6.1 $\left(m \gamma_{k}\right) \tilde{\gamma}_{j}=\left(m \tilde{\gamma}_{j}\right) \gamma_{k}$.

(3): Set $\mathbf{v}=v_{2} \otimes \cdots \otimes v_{j} \otimes v_{n} \otimes v_{j+1} \otimes \cdots \otimes v_{k-1} \otimes v_{n-1} \otimes v_{k+1} \otimes \cdots \otimes v_{l}$ and $\widetilde{\mathbf{v}}=\sigma_{j k}(\mathbf{v})$; let $\widehat{\mathbf{v}}$ be the same as $\mathbf{v}$ except that $v_{n}$ is replaced by $v_{1}$ and set $\overline{\mathbf{v}}=\sigma_{j k}(\widehat{\mathbf{v}})$. Then

$\left(m \sigma_{j k}\right) \gamma_{j} \otimes \mathbf{v}=\mathrm{E}_{n 1}\left(\gamma^{-1}\right)\left(m \sigma_{j k} \otimes \widehat{\mathbf{v}}\right)=\mathrm{E}_{n 1}\left(\gamma^{-1}\right)(m \otimes \overline{\mathbf{v}})=m \gamma_{k} \otimes \widetilde{\mathbf{v}}=\left(m \gamma_{k}\right) \sigma_{j k} \otimes \mathbf{v}$.

Again, Lemma 6.1 allows us to conclude that $\left(m \sigma_{j k}\right) \gamma_{j}=\left(m \gamma_{k}\right) \sigma_{j k}$.

Finally, one can check that the functor $F$ is bijective on sets of morphisms.

Proof of Theorem 6.1. Let $N$ be a left module over $\mathrm{D}_{\beta, \mathbf{b}}^{n}$ which is integrable and of level $l$. Proposition 6.2 says that $N=M \otimes_{\mathbb{C}\left[S_{l}\right]}\left(\mathbb{C}^{n}\right)^{\otimes l}$ for some right $\Gamma_{l}$-module $M$. We have to extend this to a right module structure over $\mathrm{H}_{t, \mathbf{c}}\left(\Gamma_{l}\right)$.

We can proceed exactly as in the proof of Proposition 6.2 (mimicking the arguments in [ChPr1] ) to show that there exist endomorphisms $\zeta_{k}^{w} \in \operatorname{End}_{\mathbb{C}}(M)$ such that $\mathrm{E}_{a b}(w)(m \otimes \mathbf{v})=\sum_{k=1}^{l} \zeta_{k}^{w}(m) \otimes E_{a b}^{(k)}(\mathbf{v})$. We proceed as in $[\mathrm{Gu} 2$ to show that setting $m x_{k}=\zeta_{k}^{u}(m), m y_{j}=\zeta_{j}^{v}(m)$ turns $M$ into a module over $\mathrm{H}_{t, \mathbf{c}}\left(\Gamma_{l}\right)$.

Fix $1 \leq j, k \leq l, j \neq k$. Choose $\mathbf{v}=v_{i_{1}} \otimes \cdots \otimes v_{i_{l}}$ such that $i_{k}=2, i_{j}=n-1$, $i_{r}=r+2$ if $r<j, r \neq k, i_{r}=r+1$ if $r>j, r \neq k$. Set $\widetilde{\mathbf{v}}=E_{n 2}^{(k)} E_{1, n-1}^{(j)}(\mathbf{v})$. On the one hand,

$$
\begin{aligned}
& \left(\mathrm{E}_{1, n-1}\left(w_{2}\right) \mathrm{E}_{n 2}\left(w_{1}\right)-\mathrm{E}_{n 2}\left(w_{1}\right) \mathrm{E}_{1, n-1}\left(w_{2}\right)\right)(m \otimes \mathbf{v}) \\
& \quad=\sum_{s=1}^{l} \sum_{r=1}^{l} m w_{r}^{1} w_{s}^{2} \otimes E_{1, n-1}^{(s)} E_{n 2}^{(r)}(\mathbf{v})-\sum_{s=1}^{l} \sum_{r=1}^{l} m w_{s}^{2} w_{r}^{1} \otimes E_{n 2}^{(r)} E_{1, n-1}^{(s)}(\mathbf{v}) \\
& \quad=m\left(w_{k}^{1} w_{j}^{2}-w_{j}^{2} w_{k}^{1}\right) \otimes \widetilde{\mathbf{v}} .
\end{aligned}
$$

Using relation (36) for $E_{a b}=E_{n 2}$ and $E_{c d}=E_{1, n-1}$, we find that

$$
\left[\mathrm{E}_{1, n-1}\left(w_{2}\right), \mathrm{E}_{n 2}\left(w_{1}\right)\right]=-\frac{\lambda}{2} \sum_{\gamma \in \Gamma} \omega\left(\gamma\left(w_{1}\right), w_{2}\right) \mathrm{E}_{n, n-1}\left(\gamma^{-1}\right) \mathrm{E}_{12}(\gamma),
$$


SO

$$
\begin{aligned}
{\left[\mathrm{E}_{1, n-1}\left(w_{2}\right), \mathrm{E}_{n 2}\left(w_{1}\right)\right](m \otimes \mathbf{v}) } & =-\frac{\lambda}{2} \sum_{\gamma \in \Gamma} \omega\left(\gamma\left(w_{1}\right), w_{2}\right) m \gamma_{k}^{-1} \gamma_{j} \otimes E_{n, n-1}^{(j)} E_{12}^{(k)}(\mathbf{v}) \\
& =-\frac{\lambda}{2} \sum_{\gamma \in \Gamma} \omega\left(\gamma\left(w_{2}\right), w_{1}\right) m \gamma_{j} \gamma_{k}^{-1} \sigma_{j k} \otimes \widetilde{\mathbf{v}}
\end{aligned}
$$

Therefore, $m\left(w_{k}^{1} w_{j}^{2}-w_{j}^{2} w_{k}^{1}+\frac{\lambda}{2} \sum_{\gamma \in \Gamma} \omega\left(\gamma\left(w_{1}\right), w_{2}\right) \sigma_{j k} \gamma_{k} \gamma_{j}^{-1}\right) \otimes \widetilde{\mathbf{v}}=0$. From Lemma 6.1 and our assumption that $\lambda=\kappa$, we deduce that $m\left(w_{k}^{1} w_{j}^{2}-w_{j}^{2} w_{k}^{1}+\right.$ $\left.\left.\frac{\kappa}{2} \sum_{\gamma \in \Gamma} \omega(\gamma(x), y)\right) \sigma_{j k} \gamma_{k} \gamma_{j}^{-1}\right)=0$.

We use equation (35) in the case $(a, b)=(n, 1),(c, d)=(n-1,1)$. It implies that the difference $\left[\mathrm{E}_{n 1}(v), \mathrm{E}_{1, n-1}(u)\right]-\left[\mathrm{E}_{n 1}(u), \mathrm{E}_{1, n-1}(v)\right]$ is equal to

$$
\begin{aligned}
\mathrm{E}_{n, n-1} & (\mathbf{b}+\beta)+\frac{\lambda}{4} \sum_{\gamma \in \Gamma} \sum_{j=1}^{n-2} S\left(\mathrm{E}_{n j}(\gamma), \mathrm{E}_{j, n-1}\left(\gamma^{-1}\right)\right) \\
& +\frac{\lambda}{4} \sum_{\gamma \in \Gamma}\left(S\left(\mathrm{H}_{n 1}(\gamma), \mathrm{E}_{n, n-1}\left(\gamma^{-1}\right)\right)+S\left(\mathrm{E}_{n, n-1}(\gamma), \mathrm{H}_{n-1,1}\left(\gamma^{-1}\right)\right)\right) \\
& -\frac{\lambda}{2} \sum_{\gamma \in \Gamma}(\omega(\gamma(u), v)-1)\left(\mathrm{E}_{11}\left(\gamma^{-1}\right) \mathrm{E}_{n, n-1}(\gamma)+\mathrm{E}_{11}(\gamma) \mathrm{E}_{n, n-1}\left(\gamma^{-1}\right)\right) .
\end{aligned}
$$

Now fix $k$ and let $\mathbf{v}$ be determined by $i_{k}=n-1, i_{j}=j+1$ if $j \neq k$. Set $\widehat{\mathbf{v}}=E_{n, n-1}^{(k)}(\mathbf{v})$. Applying both sides of the previous equality to $m \otimes \mathbf{v}$, we deduce that

$$
\begin{aligned}
m\left(x_{k} y_{k}-y_{k} x_{k}\right) \otimes \widehat{\mathbf{v}}=m\left(\beta+\lambda+\sum_{\substack{\gamma \in \Gamma \\
\gamma \neq \mathrm{id}}} b_{\gamma} \gamma^{-1}\right) & \otimes \widehat{\mathbf{v}}+\frac{\lambda n|\Gamma|}{4} m \otimes \widehat{\mathbf{v}} \\
& +\frac{\lambda}{2} \sum_{\substack { \gamma \in \Gamma \\
\begin{subarray}{c}{j, k=1 \\
j \neq k{ \gamma \in \Gamma \\
\begin{subarray} { c } { j , k = 1 \\
j \neq k } }\end{subarray}}^{n} m \sigma_{j k} \gamma_{k} \gamma_{j}^{-1} \otimes \widehat{\mathbf{v}}
\end{aligned}
$$

Lemma 6.1 and our assumption that $\lambda=\kappa, \beta=t-\frac{\kappa n|\Gamma|}{4}-\kappa, b_{\gamma}=c_{\gamma^{-1}}$ imply that $\left[x_{k}, y_{k}\right]=t+\frac{\kappa}{2} \sum_{\gamma \in \Gamma} \sum_{\substack{j, k=1 \\ j \neq k}}^{n} \sigma_{j k} \gamma_{k} \gamma_{j}^{-1}+\sum_{\substack{\gamma \in \Gamma \\ \gamma \neq \mathrm{id}}} c_{\gamma} \gamma$

That the functor SW is bijective on sets of morphisms follows from the classical Schur-Weyl duality and Lemma 6.1.

\section{Specialization at $\lambda=0$}

In $[\mathrm{Gu} 2$, we proved that, when the parameters $\lambda=0$ and $\beta \neq 0$, the deformed double current algebra is isomorphic to the enveloping algebra of the Lie algebra $\mathfrak{g l}_{n}$ over the first Weyl algebra, which is a symplectic reflection algebra of rank one for the trivial group $\Gamma=\{1\}$. Therefore, it is natural to conjecture that, for an arbitrary finite subgroup $\Gamma$ of $S L_{2}(\mathbb{C})$, a similar result is true, the first Weyl algebra being replaced by a symplectic reflection algebra of rank one for $\Gamma$. Theorem 7.1 confirms this. 
Definition 7.1 ([CBHo $)$. Set $\widetilde{\mathbf{c}}=\sum_{\gamma \in \Gamma \backslash\{\text { id }\}} c_{\gamma} \gamma$. Let $\mathrm{A}_{t, \widetilde{\mathbf{c}}}$ be the algebra generated by the elements $x, y, \gamma \in \Gamma$ and satisfying the relations $\gamma \cdot x \cdot \gamma^{-1}=\gamma(x)$, $\gamma \cdot y \cdot \gamma^{-1}=\gamma(y)$ and $x y-y x=t+\widetilde{\mathbf{c}}$. (Here, $\operatorname{span}_{\mathbb{C}}\{x, y\} \cong \mathbb{C}^{2}$ and $\Gamma$ thus acts on $\left.\operatorname{span}_{\mathbb{C}}\{x, y\}.\right)$

We recall that the Lie algebra $\mathfrak{s l}_{n}\left(\mathrm{~A}_{t, \widetilde{\mathbf{c}}}\right)$ is defined as the Lie subalgebra of $\mathfrak{g l}_{n}\left(\mathrm{~A}_{t, \tilde{\mathbf{c}}}\right)$ of matrices with trace in $\left[\mathrm{A}_{t, \tilde{\mathbf{c}}}, \mathrm{A}_{t, \tilde{\mathbf{c}}}\right]$. As a vector space, $\mathfrak{s l}_{n}\left(\mathrm{~A}_{t, \widetilde{\mathbf{c}}}\right)=$ $\mathfrak{s l}_{n} \otimes_{\mathbb{C}} A_{t, \widetilde{\mathbf{c}}} \oplus \mathfrak{d}\left(\left[\mathrm{A}_{t, \tilde{\mathbf{c}}}, A_{t, \tilde{\mathbf{c}}}\right]\right)$, where $\mathfrak{d}\left(\left[\mathrm{A}_{t, \tilde{\mathbf{c}}}, \mathrm{A}_{t, \tilde{\mathbf{c}}}\right]\right)$ is the space of scalar matrices in $\mathfrak{g l}_{n}\left(\mathrm{~A}_{t, \tilde{\mathbf{c}}}\right)$ with entries in $\left[A_{t, \tilde{\mathbf{c}}}, A_{t, \tilde{\mathbf{c}}}\right]$.

Lemma 7.1. For all $\widetilde{\mathbf{c}}$ and all $t \in \mathbb{C}^{\times}$except a countable set, the Lie algebra $\mathfrak{s l}_{n}\left(\mathrm{~A}_{t, \widetilde{\mathbf{c}}}\right)$ is universally closed; that is, it is its own universal central extension.

Proof. Theorem 1.7 in KaLo states that the center of the universal central extension of $\mathfrak{s l}_{n}(\mathcal{A})$, where $\mathcal{A}$ is an arbitrary associative algebra, is isomorphic to the first cyclic homology group $\mathrm{HC}_{1}(\mathcal{A})$. It is shown in EtGi that the first Hochschild homology group $\mathrm{HH}_{1}\left(\mathrm{~A}_{t, \widetilde{\mathbf{c}}}\right)$ vanishes for all $\widetilde{\mathbf{c}}$ and all $t \in \mathbb{C}^{\times}$outside a countable set. The group $\mathrm{HC}_{1}\left(\mathrm{~A}_{t, \tilde{\mathbf{c}}}\right)$ is a quotient of $\mathrm{HH}_{1}\left(\mathrm{~A}_{t, \tilde{\mathbf{c}}}\right)$, so it vanishes also.

Theorem 7.1. Suppose that $\beta=t$ and $b_{\gamma}=c_{\gamma^{-1}}$ for $\gamma \neq \mathrm{id}$. Then the algebra $\widetilde{\mathrm{D}}_{\beta, \widetilde{\mathbf{b}}}^{n}$ is isomorphic to the enveloping algebra of the Lie algebra $\widehat{\mathfrak{s l}}_{n}\left(\mathrm{~A}_{t, \tilde{\mathbf{c}}}\right)$, the universal central extension of $\mathfrak{s l}_{n}\left(\mathrm{~A}_{t, \tilde{\mathbf{c}}}\right)$.

Proof. It follows from the definition of $\widetilde{\mathrm{D}}_{\beta, \widetilde{\mathbf{b}}}^{n}$ and Theorem 4.1 that $\mathfrak{U}_{\mathfrak{s l}}\left(\mathrm{A}_{t, \widetilde{\mathbf{c}}}\right)$ is a quotient of $\widetilde{\mathrm{D}}_{\beta, \mathbf{b}}^{n}$. To prove that the quotient map is an isomorphism, we construct elements $\mathrm{E}_{a b}(p) \in \widetilde{\mathrm{D}}_{\beta, \widetilde{\mathbf{b}}}^{n}$ for $1 \leq a \neq b \leq n$ and any $p \in \mathrm{A}_{t, \widetilde{\mathbf{c}}}$ and show that they satisfy the relations in Theorem 4.1. We will give a proof when $n \geq 5$; it illustrates how the calculations are sometimes simpler when $n \geq 5$. Let $\mathfrak{g}$ be the Lie algebra defined by the relations in Definition 5.1 with $\lambda=0$. Lemma 3.4 gives us homomorphisms $\mathfrak{s l}_{n}[v] \longrightarrow \mathfrak{g}, \mathfrak{s l}_{n}[u] \longrightarrow \mathfrak{g}$. Define $\mathrm{E}_{a b}\left(v^{j} \gamma\right)=\left[\mathrm{E}_{a c}\left(v^{j}\right), \mathrm{E}_{c b}(\gamma)\right]$ for $\gamma \neq \mathrm{id}, a, b, c$ all distinct, and set inductively $\mathrm{E}_{a b}\left(u^{i} v^{j} \gamma\right)=\left[\mathrm{E}_{a c}(u), \mathrm{E}_{c b}\left(u^{i-1} v^{j} \gamma\right)\right]$ for some $c \neq a, b$ and for $i, j \geq 1$. We define $\mathrm{E}_{a b}(p)$ by linearity when $p$ is a sum of monomials. We have to show that $\left[\mathrm{E}_{a b}\left(p_{1}\right), \mathrm{E}_{b c}\left(p_{2}\right)\right]=\mathrm{E}_{a c}\left(p_{1} p_{2}\right)$ if $a \neq b \neq c \neq a$ and $\left[\mathrm{E}_{a b}\left(p_{1}\right), \mathrm{E}_{c d}\left(p_{2}\right)\right]=0$ if $a \neq b \neq c \neq d \neq a$ for any $p_{1}=u^{i_{1}} v^{j_{1}} \gamma_{1}, p_{2}=u^{i_{2}} v^{j_{2}} \gamma_{2}$.

The first step, however, is to show that the definition of $\mathrm{E}_{a b}\left(u^{i} v^{j} \gamma\right)$ does not depend on the choice of $c$. Since we are assuming that $n \geq 5$, choose $d, e$ such that $a, b, c, d, e$ are all distinct and assume that $i \geq 2$. (The case $i=1, j \geq 1$ is similar.) Then

$$
\begin{aligned}
{\left[\mathrm{E}_{a c}(u), \mathrm{E}_{c b}\left(u^{i-1} v^{j} \gamma\right)\right] } & =\left[\mathrm{E}_{a c}(u),\left[\mathrm{E}_{c d},\left[\mathrm{E}_{d e}(u), \mathrm{E}_{e b}\left(u^{i-2} v^{j} \gamma\right)\right]\right]\right] \\
& =\left[\left[\mathrm{E}_{a c}(u), \mathrm{E}_{c d}\right],\left[\mathrm{E}_{d e}(u), \mathrm{E}_{e b}\left(u^{i-2} v^{j} \gamma\right)\right]\right] \\
& =\left[\mathrm{E}_{a d}(u), \mathrm{E}_{d b}\left(u^{i-2} v^{j} \gamma\right)\right] .
\end{aligned}
$$

The arguments used are similar to those in the proofs of Lemmas 3.4, 3.5 and 4.1. We proceed again by induction on $\operatorname{deg}\left(p_{1}\right)+\operatorname{deg}\left(p_{2}\right)$ to prove the two equalities above, which hold when $\operatorname{deg}\left(p_{1}\right)+\operatorname{deg}\left(p_{2}\right) \leq 1$.

If $a \neq b \neq c \neq d \neq a$, choose $e \neq a, b, c, d$. Without loss of generality, we can suppose that $p_{1}=u \widetilde{p}_{1}$ and $\operatorname{deg}\left(p_{1}\right) \geq 2$. Then $\left[\mathrm{E}_{a b}\left(p_{1}\right), \mathrm{E}_{c d}\left(p_{2}\right)\right]=\left[\left[\mathrm{E}_{a e}(u), \mathrm{E}_{e b}\left(\widetilde{p}_{1}\right)\right]\right.$, $\left.\mathrm{E}_{c d}\left(p_{2}\right)\right]=0$ by induction. 
If $p_{1}=u \widetilde{p}_{1}$ with $\operatorname{deg}\left(\widetilde{p}_{1}\right) \geq 1$, choose $a, b, c, d$ all distinct, so that, by induction,

$$
\begin{aligned}
{\left[\mathrm{E}_{a b}\left(p_{1}\right), \mathrm{E}_{b c}\left(p_{2}\right)\right] } & \left.=\left[\left[\mathrm{E}_{a d}(u), \mathrm{E}_{d b}\left(\widetilde{p}_{1}\right)\right], \mathrm{E}_{b c}\left(p_{2}\right)\right]=\left[\mathrm{E}_{a d}(u),\left[\mathrm{E}_{d b}\left(\widetilde{p}_{1}\right)\right], \mathrm{E}_{b c}\left(p_{2}\right)\right]\right] \\
& =\left[\mathrm{E}_{a d}(u), \mathrm{E}_{d c}\left(\widetilde{p}_{1} p_{2}\right)\right]=\mathrm{E}_{a c}\left(u \widetilde{p}_{1} p_{2}\right)=\mathrm{E}_{a c}\left(p_{1} p_{2}\right) .
\end{aligned}
$$

If $p_{1}=v^{r}$ with $r \geq 1$ and $p_{2}=u \widetilde{p}_{2}$, then choose $a, b, c, d, e$ all distinct, so that

$$
\begin{aligned}
& {\left[\mathrm{E}_{a b}\left(p_{1}\right), \mathrm{E}_{b c}\left(p_{2}\right)\right] } \\
= & {\left[\left[\mathrm{E}_{a d}(v), \mathrm{E}_{d b}\left(v^{r-1}\right], \mathrm{E}_{b c}\left(p_{2}\right)\right]=\left[\mathrm{E}_{a d}(v),\left[\mathrm{E}_{d b}\left(v^{r-1}\right), \mathrm{E}_{b c}\left(p_{2}\right)\right]\right]\right.} \\
= & {\left[\mathrm{E}_{a d}(v), \mathrm{E}_{d c}\left(v^{r-1} p_{2}\right)\right]=\left[\mathrm{E}_{a d}(v), \mathrm{E}_{d c}\left(u v^{r-1} \widetilde{p}_{2}\right)+\mathrm{E}_{d c}\left(\left[v^{r-1}, u\right] \widetilde{p}_{2}\right)\right] } \\
= & {\left[\mathrm{E}_{a d}(v),\left[\mathrm{E}_{d e}(u), \mathrm{E}_{e c}\left(v^{r-1} \widetilde{p}_{2}\right)\right]\right]+\left[\mathrm{E}_{a d}(v), \mathrm{E}_{d c}\left(\left[v^{r-1}, u\right] \widetilde{p}_{2}\right)\right] } \\
= & {\left[\left[\mathrm{E}_{a d}(v), \mathrm{E}_{d e}(u)\right], \mathrm{E}_{e c}\left(v^{r-1} \widetilde{p}_{2}\right)\right]+\mathrm{E}_{a c}\left(v\left[v^{r-1}, u\right] \widetilde{p}_{2}\right) } \\
= & {\left[\left[\mathrm{E}_{a d}(u), \mathrm{E}_{d e}(v)\right], \mathrm{E}_{e c}\left(v^{r-1} \widetilde{p}_{2}\right)\right]-\left[\mathrm{E}_{a e}(\widetilde{\mathbf{c}}), \mathrm{E}_{e c}\left(v^{r-1} \widetilde{p}_{2}\right)\right]+\mathrm{E}_{a c}\left(v\left[v^{r-1}, u\right] \widetilde{p}_{2}\right) } \\
= & \mathrm{E}_{a c}\left(u v^{r} \widetilde{p}_{2}\right)-\mathrm{E}_{a c}\left(\widetilde{\mathbf{c}} v^{r-1} \widetilde{p}_{2}\right)+\mathrm{E}_{a c}\left(v\left[v^{r-1}, u\right] \widetilde{p}_{2}\right) \\
= & \mathrm{E}_{a c}\left(p_{1} p_{2}\right) .
\end{aligned}
$$

If $p_{1}=\widetilde{p}_{1} \gamma$ with $\widetilde{p}_{1}$ a monomial in $u, v, p_{2}=u \widetilde{p}_{2}$ and $a, b, c, d, e$ are all distinct, then

$$
\begin{aligned}
{\left[\mathrm{E}_{a b}\left(p_{1}\right), \mathrm{E}_{b c}\left(p_{2}\right)\right] } & =\left[\left[\mathrm{E}_{a e}\left(\widetilde{p}_{1}\right), \mathrm{E}_{e b}(\gamma)\right],\left[\mathrm{E}_{b d}(u), \mathrm{E}_{d c}\left(\widetilde{p}_{2}\right)\right]\right] \\
& =\left[\mathrm{E}_{a e}\left(\widetilde{p}_{1}\right),\left[\left[\mathrm{E}_{e b}(\gamma), \mathrm{E}_{b d}(u)\right], \mathrm{E}_{d c}\left(\widetilde{p}_{2}\right)\right]\right] \\
& =\left[\mathrm{E}_{a e}\left(\widetilde{p}_{1}\right),\left[\left[\mathrm{E}_{e b}(\gamma(u)), \mathrm{E}_{b d}(\gamma)\right], \mathrm{E}_{d c}\left(\widetilde{p}_{2}\right)\right]\right] \\
& =\left[\mathrm{E}_{a e}\left(\widetilde{p}_{1}\right), \mathrm{E}_{e c}\left(\gamma\left(u \widetilde{p}_{2}\right) \gamma\right)\right] \\
& =\left[\mathrm{E}_{a e}\left(\widetilde{p}_{1}\right), \mathrm{E}_{e c}\left(\gamma\left(p_{2}\right) \gamma\right)\right]=\mathrm{E}_{a c}\left(p_{1} p_{2}\right) .
\end{aligned}
$$

The last line follows from the previous cases since $\widetilde{p}_{1}$ is assumed to be a monomial in $u, v$.

Corollary 7.1. For all $\mathbf{b}(\lambda=0)$ and all $\beta \in \mathbb{C}^{\times}$outside a countable set, the algebra $\mathrm{D}_{\beta, \mathbf{b}(\lambda=0)}^{n}$ is isomorphic to the enveloping algebra of the Lie algebra $\mathfrak{g l}_{n}\left(\mathrm{~A}_{t, \tilde{\mathbf{c}}}\right)$ with $t=\beta, c_{\gamma}=b_{\gamma^{-1}}$ for $\gamma \neq \mathrm{id}$. This is true, in particular, when $\lambda=0=b_{\gamma}$ for $\gamma \neq \mathrm{id}$ and $\beta \neq 0$.

Proof. Lemma 7.1 and Theorem 7.1 imply that $\widetilde{\mathrm{D}}_{\beta, \widetilde{\mathbf{b}}}^{n}$ is isomorphic to $\mathfrak{s l}_{n}\left(\mathrm{~A}_{t, \widetilde{\mathbf{c}}}\right)$ with $t=\beta, c_{\gamma}=b_{\gamma^{-1}}$ for $\gamma \neq$ id. The isomorphism given in Theorem 7.1 can be extended to $\mathrm{D}_{\beta, \mathbf{b}(\lambda=0)}^{n}$ and $\mathfrak{g l}_{n}\left(\mathrm{~A}_{t, \widetilde{\mathbf{c}}}\right)$ by sending $\mathrm{E}_{a a}(\gamma)$ to $E_{a a} \otimes \gamma$ for $\gamma \neq$ id. Note that $\frac{\mathfrak{g l}_{n}\left(\mathrm{~A}_{t, \tilde{\mathbf{c}}}\right)}{\mathfrak{s} \mathfrak{l}_{n}\left(\mathrm{~A}_{t, \tilde{\mathbf{c}}}\right)} \cong \frac{\mathrm{A}_{t, \tilde{\mathbf{c}}}}{\left[\mathrm{A}_{t, \tilde{\mathbf{c}}}, \mathrm{A}_{t, \tilde{\mathbf{c}}}\right]}=\mathrm{HC}_{0}\left(\mathrm{~A}_{t, \widetilde{\mathbf{c}}}\right)$, and it is proved in [EtGi] that $\operatorname{dim}_{\mathbb{C}}\left(\mathrm{A}_{t, \widetilde{\mathbf{c}}}\right)=$ $c l(\Gamma)-1$ for generic values of the parameters (in the same sense as above).

\section{PBW BASES}

We follow the same approach as in Gu2 to prove that a $\Gamma$-deformed double current algebra admits a vector space basis of PBW type. This can be formulated by saying that the map $\mathrm{D}_{\beta=0, \mathbf{b}=\mathbf{0}}^{n} \rightarrow \operatorname{gr}_{F}\left(\mathrm{D}_{\beta, \mathbf{b}}^{n}\right)$ is an isomorphism. We will construct inductively a vector space basis of $\mathrm{D}_{\beta, \mathbf{b}}^{n}$ which yields the natural PBW basis on $\operatorname{gr}_{F}\left(\mathrm{D}_{\beta, \mathbf{b}}^{n}\right) \cong \mathfrak{U}_{\mathfrak{s}} l_{n}(\mathbb{C}[u, v] \rtimes \Gamma)$. We make the same assumption on $u, v, x, y$ as in section 6. We need to assume that $\beta+\lambda-\frac{\lambda n|\Gamma|}{4} \neq 0$ in this section. 
We need to choose in $\mathbb{C}[u, v]$ a $\Gamma$-invariant space $E$ complementary to $\mathbb{C}[u, v]^{\Gamma}$, so that $\mathbb{C}[u, v] \cong \mathbb{C}[u, v]^{\Gamma} \oplus E$ as $\Gamma$-modules. We can suppose that $E=\bigoplus_{m>1} E[m]$ is graded by the degree of the monomials and $E(1)=U$. Let us assume that we have constructed elements $\mathrm{F}_{a b}(p) \in \mathrm{D}_{\beta, \mathbf{b}}^{n}$ for all $p \in E \oplus \operatorname{span}\{\widetilde{p} \gamma \mid \widetilde{p} \in \mathbb{C}[u, v], \gamma \neq \mathrm{id}\}$ of degree $\leq N \forall 1 \leq a, b \leq n$ and also $\forall p \in \mathbb{C}[u, v]^{\Gamma}+\mathbb{C}[\Gamma]$ of degree $\leq N-2$ $\forall 1 \leq a, b \leq n$, such that $\mathrm{F}_{a b}(p)(\mathbf{h} \otimes \mathbf{v})=\sum_{k=1}^{l} \mathrm{~h} p\left(x_{k}, y_{k}, \gamma_{k}\right) \otimes E_{a b}^{(k)}(\mathbf{v})$ if $\mathbf{h} \in$ $\mathrm{H}_{t, \mathbf{c}}\left(\Gamma_{l}\right), \mathbf{v} \in\left(\mathbb{C}^{n}\right)^{\otimes l}$. This is already known to hold for $N=1$. We use the notation $\mathrm{F}_{a b}(p)$ instead of $\mathrm{E}_{a b}(p)$ because we must set $\mathrm{F}_{a b}(\gamma)=\mathrm{E}_{a b}\left(\gamma^{-1}\right), \mathrm{F}_{a b}\left(t_{1} u+t_{2} v\right)=$ $\mathrm{E}_{a b}\left(t_{1} u+t_{2} v\right), t_{1}, t_{2} \in \mathbb{C}$ and $\mathrm{F}_{a b}(w \gamma)=\left[\mathrm{E}_{a c}\left(\gamma^{-1}\right), \mathrm{E}_{c b}(w)\right]$ for some $c \neq a, b$. We want to construct by induction such elements $\mathrm{F}_{a b}(p)$ for any $p \in \mathbb{C}[u, v] \rtimes \Gamma, 1 \leq$ $a, b \leq n$. Set $\overline{\mathrm{H}}_{a b}(p)=\left[\mathrm{F}_{a b}(p), \mathrm{F}_{b a}\right]$ for $1 \leq a \neq b \leq n$ if $\mathrm{F}_{a b}(p)$ has already been defined.

Let $p(u, v) \in \mathbb{C}[u, v]^{\Gamma}, p(u, v) \neq 0$ be a polynomial of degree $N-1$ (we can assume that $p(u, v)$ is homogeneous). In the computations below for $\widehat{P}(\mathbf{h} \otimes \mathbf{v})$, we will not need to use that $p(u, v)$ is $\Gamma$-invariant. However, we have to start with this case in the induction step.

Suppose that $p(u, v)=\sum_{r, s \geq 0}\left(c_{r, s}^{p} r u^{s} v^{r-1}-\tilde{c}_{r, s}^{p} s u^{s-1} v^{r}\right)$, where $c_{r, s}^{p}, \tilde{c}_{r, s}^{p} \in \mathbb{C}$ and $c_{r, s}^{p}=0=\widetilde{c}(p, r, s)$ if $r+s \neq f$. The proof of Proposition 4.2 suggests that we consider the following elements:

$$
\begin{aligned}
& \widehat{P}_{i}=\sum_{r, s \geq 0}\left(c_{r, s}^{p}\left[\overline{\mathrm{H}}_{i}\left(u^{s} v^{r}\right), \overline{\mathrm{H}}_{i}(u)\right]+\tilde{c}_{r, s}^{p}\left[\overline{\mathrm{H}}_{i}\left(u^{s} v^{r}\right), \overline{\mathrm{H}}_{i}(v)\right]\right) \text { and } \widehat{P}=\frac{1}{2} \sum_{i=1}^{n} \widehat{P}_{i} . \\
& \widehat{P}(\mathrm{~h} \otimes \mathbf{v})=\frac{1}{2} \sum_{i=1}^{n} \sum_{r, s \geq 0} \sum_{k=1}^{l}\left(c_{r, s}^{p} \mathrm{~h}\left[x_{k}, x_{k}^{s} y_{k}^{r}\right]-\tilde{c}_{r, s}^{p} \mathrm{~h}\left[x_{k}^{s} y_{k}^{r}, y_{k}\right]\right) \\
&+\frac{1}{2} \sum_{i=1}^{n} \sum_{r, s \geq 0} \sum_{\substack{j, k=1 \\
j \neq k}}^{l}\left(c_{r, s}^{p} \mathrm{~h}\left[x_{k}, x_{j}^{s} y_{j}^{r}\right]-\tilde{c}_{r, s}^{p} \mathrm{~h}\left[x_{j}^{s} y_{j}^{r}, y_{k}\right]\right) \otimes H_{i}^{(j)} H_{i}^{(k)} \mathbf{v} \\
&=\sum_{r, s \geq 0} \sum_{k=1}^{l}\left(c_{r, s}^{p} \sum_{d=0}^{r-1} \mathrm{~h} x_{k}^{s} y_{k}^{d}\left[x_{k}, y_{k}\right] y_{k}^{r-d-1}-\tilde{c}_{r, s}^{p} \sum_{d=0}^{s-1} \mathrm{~h} x_{k}^{d}\left[x_{k}, y_{k}\right] x_{k}^{s-d-1} y_{k}^{r}\right) \otimes \mathbf{v} \\
&+\sum_{i=1}^{n} \sum_{r, s \geq 0} \sum_{d=0}^{s-1} \sum_{\substack{d, k=1 \\
j \neq k}}^{l}\left(\frac{c_{r, s}^{p}}{2} \mathrm{~h} x_{j}^{d}\left[x_{k}, x_{j}\right] x_{j}^{s-d-1} y_{j}^{r}-\frac{\tilde{c}_{r, s}^{p}}{2} \mathrm{~h} x_{j}^{d}\left[x_{j}, y_{k}\right] x_{j}^{s-d-1} y_{j}^{r}\right) \\
&+\sum_{i=1}^{n} \sum_{r, s \geq 0} \sum_{\substack{j, k=1 \\
j \neq k}}^{l} \sum_{d=0}^{r-1}\left(\frac{c_{r, s}^{p}}{2} \mathrm{~h} x_{j}^{s} y_{j}^{d}\left[x_{k}, y_{j}\right] y_{j}^{r-d-1}-\frac{\tilde{c}_{r, s}^{p}}{2} \mathrm{~h} x_{j}^{s} y_{j}^{d}\left[y_{j}, y_{k}\right] y_{j}^{r-d-1}\right) \\
&=t \sum_{r, s \geq 0} \sum_{k=1}^{l}\left(c_{r, s}^{p} r \mathrm{~h} x_{k}^{s} y_{k}^{r-1}-\tilde{c}_{r, s}^{p} s \mathrm{~h} x_{k}^{s-1} y_{k}^{r}\right) \otimes \mathbf{v} \\
& H_{i}^{(k)} H_{i}^{(j)} \mathbf{v}
\end{aligned}
$$




$$
\begin{aligned}
& +\sum_{r, s \geq 0} c_{r, s}^{p} \sum_{d=0}^{r-1}\left(\frac{\kappa}{2} \sum_{\substack{j, k=1 \\
j \neq k}}^{l} \sum_{\gamma \in \Gamma} \mathrm{h} x_{k}^{s} y_{k}^{d} \sigma_{j k} \gamma_{k} \gamma_{j}^{-1} y_{k}^{r-d-1}\right. \\
& \left.+\sum_{\gamma \in \Gamma \backslash\{\mathrm{id}\}} c_{\gamma} \mathrm{h} x_{k}^{s} y_{k}^{d} \gamma_{k} y_{k}^{r-d-1}\right) \otimes \mathbf{v} \\
& -\sum_{r, s \geq 0} \tilde{c}_{r, s}^{p} \sum_{d=0}^{s-1}\left(\frac{\kappa}{2} \sum_{\substack{j, k=1 \\
j \neq k}}^{l} \sum_{\gamma \in \Gamma} \mathrm{h} x_{k}^{d} \sigma_{j k} \gamma_{k} \gamma_{j}^{-1} x_{k}^{s-d-1} y_{k}^{r}\right. \\
& \left.+\sum_{\gamma \in \Gamma \backslash\{\mathrm{id}\}} c_{\gamma} \mathrm{h} x_{k}^{d} \gamma_{k} x_{k}^{s-d-1} y_{k}^{r}\right) \otimes \mathbf{v} \\
& -\frac{\kappa}{4} \sum_{i=1}^{n} \sum_{r, s \geq 0} \sum_{j \neq k} \sum_{d=0}^{s-1} \sum_{\gamma \in \Gamma} \mathrm{h}\left(\omega_{\gamma}^{x} c_{r, s}^{p} x_{j}^{d} \sigma_{j k} \gamma_{k} \gamma_{j}^{-1} x_{j}^{s-d-1} y_{j}^{r}\right. \\
& \left.-\tilde{c}_{r, s}^{p} \omega_{\gamma}^{x, y} x_{j}^{d} \sigma_{j k} \gamma_{j} \gamma_{k}^{-1} x_{j}^{s-d-1} y_{j}^{r}\right) \otimes H_{i}^{(k)} H_{i}^{(j)}(\mathbf{v}) \\
& -\frac{\kappa}{4} \sum_{i=1}^{n} \sum_{r, s \geq 0} \sum_{j \neq k} \sum_{d=0}^{r-1} \sum_{\gamma \in \Gamma} \mathrm{h}\left(\omega_{\gamma}^{x, y} c_{r, s}^{p} x_{j}^{s} y_{j}^{d} \sigma_{j k} \gamma_{k} \gamma_{j}^{-1} y_{j}^{r-d-1}\right. \\
& \left.-\omega_{\gamma}^{y} \tilde{c}_{r, s}^{p} x_{j}^{s} y_{j}^{d} \sigma_{k j} \gamma_{j} \gamma_{k}^{-1} y_{j}^{r-d-1}\right) \otimes H_{i}^{(k)} H_{i}^{(j)}(\mathbf{v}) \\
& =t \sum_{k=1}^{l} \mathrm{~h} p\left(x_{k}, y_{k}\right) \otimes \mathbf{v} \\
& +\sum_{r, s \geq 0} \sum_{\gamma \in \Gamma \backslash\{\mathrm{id}\}} c_{\gamma}\left(c_{r, s}^{p} \sum_{d=0}^{r-1} \mathrm{~h} x_{k}^{s} y_{k}^{d} \gamma\left(y_{k}^{r-d-1}\right) \gamma_{k}\right. \\
& \left.-\tilde{c}_{r, s}^{p} \sum_{d=0}^{s-1} \mathrm{~h} x_{k}^{d} \gamma\left(x_{k}^{s-d-1} y_{k}^{r}\right) \gamma_{k}\right) \otimes \mathbf{v} \\
& +\frac{\kappa}{2} \sum_{r, s \geq 0} \sum_{\substack{j, k=1 \\
j \neq k}}^{l} \sum_{\gamma \in \Gamma} \sum_{e, i=1}^{n}\left(c_{r, s}^{p} \sum_{d=0}^{r-1} \mathrm{~h} x_{k}^{s} y_{k}^{d} \gamma\left(y_{j}^{r-d-1}\right) \gamma_{j} \gamma_{k}^{-1}\right. \\
& \left.-\tilde{c}_{r, s}^{p} \sum_{d=0}^{s-1} \mathrm{~h} x_{k}^{d} \gamma\left(x_{j}^{s-d-1} y_{j}^{r}\right) \gamma_{j} \gamma_{k}^{-1}\right) \otimes E_{e i}^{(k)} E_{i e}^{(j)}(\mathbf{v}) \\
& -\frac{\kappa}{2} \sum_{r, s \geq 0} \sum_{\substack{j, k=1 \\
j \neq k}}^{l} \sum_{d=0}^{s-1} \sum_{\gamma \in \Gamma} \sum_{i=1}^{n}\left(c_{r, s}^{p} \omega_{\gamma}^{x}-\tilde{c}_{r, s}^{p} \omega_{\gamma^{-1}}^{x, y}\right) \mathrm{h} x_{j}^{d} \gamma^{-1}\left(x_{k}^{s-d-1} y_{k}^{r}\right) \gamma_{j} \gamma_{k}^{-1} \\
& \otimes\left(E_{i i}^{(j)} E_{i i}^{(k)}-\frac{1}{2} E_{i, i+1}^{(j)} E_{i+1, i}^{(k)}-\frac{1}{2} E_{i+1, i}^{(j)} E_{i, i+1}^{(k)}\right)(\mathbf{v}) \\
& -\frac{\kappa}{2} \sum_{r, s \geq 0} \sum_{\substack{j, k=1 \\
j \neq k}} \sum_{d=0}^{r-1} \sum_{\gamma \in \Gamma} \sum_{i=1}^{n}\left(c_{r, s}^{p} \omega_{\gamma}^{x, y}-\tilde{c}_{r, s}^{p} \omega_{\gamma^{-1}}^{y}\right) \mathrm{h} x_{j}^{s} y_{j}^{d} \gamma^{-1}\left(y_{k}^{r-d-1}\right) \gamma_{j} \gamma_{k}^{-1}
\end{aligned}
$$




$$
\begin{aligned}
& \otimes\left(E_{i i}^{(j)} E_{i i}^{(k)}-\frac{1}{2} E_{i, i+1}^{(j)} E_{i+1, i}^{(k)}-\frac{1}{2} E_{i+1, i}^{(j)} E_{i, i+1}^{(k)}\right)(\mathbf{v}) \\
& =t \sum_{k=1}^{l} \mathrm{~h} p\left(x_{k}, y_{k}\right) \otimes \mathbf{v}-\frac{\kappa n}{2} \sum_{r, s \geq 0} \sum_{d=0}^{r-1} \sum_{\gamma \in \Gamma} \sum_{k=1}^{l} c_{r, s}^{p} \mathrm{~h} x_{k}^{s} y_{k}^{r-1} \otimes \mathbf{v} \\
& +\frac{\kappa}{2} \sum_{r, s \geq 0} \sum_{d=0}^{r-1} \sum_{\gamma \in \Gamma} \sum_{\substack{e, i=1 \\
e \neq i}}^{n} c_{r, s}^{p} \mathrm{~F}_{i e}\left(\gamma\left(v^{r-d-1}\right) \gamma\right) \mathrm{F}_{e i}\left(u^{s} v^{d} \gamma^{-1}\right)(\mathbf{h} \otimes \mathbf{v}) \\
& +\sum_{r, s \geq 0} \sum_{d=0}^{r-1} \sum_{\gamma \in \Gamma \backslash\{\mathrm{id}\}} \sum_{i=1}^{n} c_{\gamma}\left(c_{r, s}^{p} \mathrm{~F}_{i i}\left(u^{s} v^{d} \gamma\left(v^{r-d-1}\right) \gamma\right)\right. \\
& \left.+\tilde{c}_{r, s}^{p} \mathrm{~F}_{i i}\left(u^{d} \gamma\left(u^{s-d-1} v^{r}\right) \gamma\right)\right)(\mathbf{h} \otimes \mathbf{v}) \\
& \left.+\frac{\kappa}{2} \sum_{r, s \geq 0} \sum_{d=0}^{r-1} \sum_{\gamma \in \Gamma \backslash\{\mathrm{id}\}} \sum_{i=1}^{n} c_{r, s}^{p}\left(1-\omega_{\gamma^{-1}}^{x, y}\right) \mathrm{F}_{i i}\left(\gamma\left(v^{r-d-1}\right) \gamma\right), \mathrm{F}_{i i}\left(u^{s} v^{d} \gamma^{-1}\right)\right)(\mathbf{h} \otimes \mathbf{v}) \\
& -\frac{\kappa}{2} \sum_{r, s \geq 0} \sum_{d=0}^{s-1} \sum_{\gamma \in \Gamma} \sum_{\substack{e, i=1 \\
e \neq i}}^{n} \tilde{c}_{r, s}^{p} \mathrm{~F}_{i e}\left(\gamma\left(u^{s-d-1} v^{r}\right) \gamma\right) \mathrm{F}_{e i}\left(u^{d} \gamma^{-1}\right)(\mathbf{h} \otimes \mathbf{v}) \\
& +\frac{\kappa n}{2} \sum_{r, s \geq 0} \sum_{d=0}^{s-1} \sum_{\gamma \in \Gamma} \sum_{k=1}^{n} \tilde{c}_{r, s}^{p} \mathrm{~h} x_{k}^{s-1} y_{k}^{r} \otimes \mathbf{v} \\
& -\frac{\kappa}{2} \sum_{r, s \geq 0} \sum_{d=0}^{s-1} \sum_{\gamma \in \Gamma \backslash\{\text { id }\}} \sum_{i=1}^{n} \tilde{c}_{r, s}^{p}\left(1-\omega_{\gamma}^{x, y}\right) \mathrm{F}_{i i}\left(\gamma\left(u^{s-d-1} v^{r}\right) \gamma\right) \mathrm{F}_{i i}\left(u^{d} \gamma^{-1}\right)(\mathbf{h} \otimes \mathbf{v}) \\
& -\frac{\kappa}{2} \sum_{r, s \geq 0} \sum_{d=0}^{s-1} \sum_{\gamma \in \Gamma \backslash\{\operatorname{id}\}} \sum_{i=1}^{n} c_{r, s}^{p} \omega_{\gamma^{-1}}^{x} \mathrm{~F}_{i i}\left(\gamma\left(u^{s-d-1} v^{r}\right) \gamma\right) \mathrm{F}_{i i}\left(u^{d} \gamma^{-1}\right)(\mathrm{h} \otimes \mathbf{v}) \\
& +\frac{\kappa}{4} \sum_{r, s \geq 0} \sum_{d=0}^{s-1} \sum_{\gamma \in \Gamma} \sum_{i=1}^{n}\left(c_{r, s}^{p} \omega_{\gamma^{-1}}^{x}-\tilde{c}_{r, s}^{p} \omega_{\gamma}^{x, y}\right)\left(\mathrm{F}_{i+1, i}\left(\gamma\left(u^{s-d-1} v^{r}\right) \gamma\right) \mathrm{F}_{i, i+1}\left(u^{d} \gamma^{-1}\right)\right. \\
& \left.+\mathrm{F}_{i, i+1}\left(\gamma\left(u^{s-d-1} v^{r}\right) \gamma\right) \mathrm{F}_{i+1, i}\left(u^{d} \gamma^{-1}\right)\right)(\mathbf{h} \otimes \mathbf{v}) \\
& +\frac{\kappa}{2} \sum_{r, s \geq 0} \sum_{d=0}^{r-1} \sum_{\gamma \in \Gamma \backslash\{\mathrm{id}\}} \sum_{i=1}^{n} \tilde{c}_{r, s}^{p} \omega_{\gamma}^{y} \mathrm{~F}_{i i}\left(\gamma\left(v^{r-d-1}\right) \gamma\right) \mathrm{F}_{i i}\left(u^{s} v^{d} \gamma^{-1}\right)(\mathbf{h} \otimes \mathbf{v}) \\
& +\frac{\kappa}{4} \sum_{r, s \geq 0} \sum_{d=0}^{r-1} \sum_{\gamma \in \Gamma} \sum_{i=1}^{n}\left(c_{r, s}^{p} \omega_{\gamma^{-1}}^{x, y}-\tilde{c}_{r, s}^{p} \omega_{\gamma}^{y}\right)\left(\mathrm{F}_{i+1, i}\left(\gamma\left(v^{r-d-1}\right) \gamma\right) \mathrm{F}_{i, i+1}\left(u^{s} v^{d} \gamma^{-1}\right)\right. \\
& \left.+\mathrm{F}_{i, i+1}\left(\gamma\left(v^{r-d-1}\right) \gamma\right) \mathrm{F}_{i+1, i}\left(u^{s} v^{d} \gamma^{-1}\right)\right)(\mathbf{h} \otimes \mathbf{v}) .
\end{aligned}
$$

Set $\widetilde{I}(p)=\widehat{P}-(37)^{\prime}-(\underline{38})^{\prime}-\cdots-(45)^{\prime}$, where (37) is the expression on line (37) but without $\mathrm{h} \otimes \mathbf{v}$, and $\mathrm{I}(p)=\left(\beta+\lambda-\frac{n \lambda|\Gamma|}{4}\right)^{-1} \widetilde{\mathrm{I}}(p)$. Then $\mathrm{I}(p)(\mathrm{h} \otimes \mathbf{v})=$ $\sum_{k=1}^{l} \mathrm{~h} p\left(x_{k}, y_{k}\right) \otimes \mathbf{v}$. 
Finally, set $\mathrm{F}_{11}(p)=\frac{1}{n} \mathrm{l}(p)+\sum_{i=1}^{n-1}\left(1-\frac{i}{n}\right) \overline{\mathrm{H}}_{i, i+1}(p)$, so $\mathrm{F}_{11}(p)(\mathbf{h} \otimes \mathbf{v})=$ $\sum_{k=1}^{l} \mathrm{~h} p\left(x_{k}, y_{k}\right) \otimes E_{11}^{(k)}(\mathbf{v})$. We can then obtain $\mathrm{F}_{i i}(p)$ with the same property. We let $\mathrm{F}_{a b}(p)=\left[\mathrm{F}_{a a}(p), \mathrm{F}_{a b}\right]$ if $1 \leq a \neq b \leq n$.

We must now construct elements $\mathrm{F}_{a b}(p) \forall p \in E \oplus \operatorname{span}\{\tilde{p} \gamma \mid \widetilde{p} \in \mathbb{C}[u, v], \gamma \neq \mathrm{id}\}$ of degree $N+1$ and $\forall: 1 \leq a, b \leq n$. Suppose first that $1 \leq a \neq b \leq n$ and put $p=u^{s} v^{r} \gamma$ with $r+s=N+1$ and, without loss of generality, $r \geq 1$. Choose $c \neq a, b$ and set $\widetilde{\mathrm{F}}_{a b}\left(u^{s} v^{r}\right)=\left[\mathrm{F}_{a c}(v), \mathrm{F}_{c b}\left(u^{s} v^{r-1}\right)\right]$. We compute that $\widetilde{\mathrm{F}}_{a b}\left(u^{s} v^{r}\right)(\mathrm{h} \otimes \mathbf{v})$ equals

$$
\begin{aligned}
& \sum_{k=1}^{l} \mathrm{~h} x_{k}^{s} y_{k}^{r} \otimes E_{a b}^{(k)}(\mathbf{v})+\sum_{\substack{j, k=1 \\
j \neq k}}^{l} \mathrm{~h}\left[x_{k}^{s} y_{k}^{r-1}, y_{j}\right] \otimes E_{a c}^{(j)} E_{c b}^{(k)}(\mathbf{v}) \\
& =\sum_{k=1}^{l} \mathrm{~h} x_{k}^{s} y_{k}^{r} \otimes E_{a b}^{(k)}(\mathbf{v})+\sum_{\substack{j, k=1 \\
j \neq k}}^{l} \sum_{d=0}^{s-1} \mathrm{~h} x_{k}^{d}\left[x_{k}, y_{j}\right] x_{k}^{s-1-d} y_{k}^{r-1} \otimes E_{a c}^{(j)} E_{c b}^{(k)}(\mathbf{v}) \\
& +\sum_{\substack{j, k=1 \\
j \neq k}}^{l} \sum_{d=0}^{r-2} \mathrm{~h} x_{k}^{s} y_{k}^{d}\left[y_{k}, y_{j}\right] y_{k}^{r-2-d} \otimes E_{a c}^{(j)} E_{c b}^{(k)}(\mathbf{v}) \\
& =\sum_{k=1}^{l} \mathrm{~h} x_{k}^{s} y_{k}^{r} \otimes E_{a b}^{(k)}(\mathbf{v})-\frac{\kappa}{2} \sum_{\substack{j, k=1 \\
j \neq k}}^{l} \sum_{d=0}^{s-1} \sum_{\gamma \in \Gamma} \omega_{\gamma}^{x, y} \mathrm{~h} x_{k}^{d} \sigma_{j k} \gamma_{k} \gamma_{j}^{-1} x_{k}^{s-1-d} y_{k}^{r-1} \otimes E_{a c}^{(j)} E_{c b}^{(k)}(\mathbf{v}) \\
& -\frac{\kappa}{2} \sum_{\substack{j, k=1 \\
j \neq k}}^{l} \sum_{d=0}^{r-2} \sum_{\gamma \in \Gamma} \omega_{\gamma}^{y} \mathrm{~h} x_{k}^{s} y_{k}^{d} \sigma_{j k} \gamma_{k} \gamma_{j}^{-1} y_{k}^{r-2-d} \otimes E_{a c}^{(j)} E_{c b}^{(k)}(\mathbf{v}) \\
& =\sum_{k=1}^{l} \mathrm{~h} x_{k}^{s} y_{k}^{r} \otimes E_{a b}^{(k)}(\mathbf{v})-\frac{\kappa}{2} \sum_{\substack{j, k=1 \\
j \neq k}}^{l} \sum_{d=0}^{s-1} \sum_{\gamma \in \Gamma} \omega_{\gamma}^{x, y} \mathrm{~h} x_{k}^{d} \gamma(x)_{j}^{s-1-d} \gamma(y)_{j}^{r-1} \gamma_{j} \gamma_{k}^{-1} \\
& \otimes E_{c c}^{(j)} E_{a b}^{(k)}(\mathbf{v})-\frac{\kappa}{2} \sum_{\substack{j, k=1 \\
j \neq k}}^{l} \sum_{d=0}^{r-2} \sum_{\gamma \in \Gamma} \omega_{\gamma}^{y} \mathrm{~h} x_{k}^{s} y_{k}^{d} \gamma(y)_{j}^{r-2-d} \gamma_{j} \gamma_{k}^{-1} \otimes E_{c c}^{(j)} E_{a b}^{(k)}(\mathbf{v}) \\
& =\sum_{k=1}^{l} \mathrm{~h} x_{k}^{s} y_{k}^{r} \otimes E_{a b}^{(k)}(\mathbf{v}) \\
& -\frac{\kappa}{2} \sum_{\gamma \in \Gamma}\left(\sum_{d=0}^{s-1} \omega_{\gamma}^{x, y} \mathrm{~F}_{c c}\left(\gamma(u)^{s-1-d} \gamma(v)^{r-1} \gamma\right) \mathrm{F}_{a b}\left(u^{d} \gamma^{-1}\right)\right. \\
& \left.+\sum_{d=0}^{r-2} \omega_{\gamma}^{y} \mathrm{~F}_{a b}\left(u^{s} v^{d} \gamma^{-1}\right), \mathrm{F}_{c c}\left(\gamma(v)^{r-2-d} \gamma\right)\right)(\mathbf{h} \otimes \mathbf{v}) .
\end{aligned}
$$

Setting $\mathrm{F}_{a b}\left(u^{s} v^{r}\right)=\widetilde{\mathrm{F}}_{a b}\left(u^{s} v^{r}\right)-(46)^{\prime}-(47)^{\prime}$, where $(46)^{\prime}$ is the expression on line (46) but without $(\mathbf{h} \otimes \mathbf{v})$, we have obtained an element with the required property. For $\gamma \neq \mathrm{id}$, we can set $\mathrm{F}_{a b}\left(u^{s} v^{r} \gamma\right)=\left[\mathrm{E}_{a a}\left(\gamma^{-1}\right), \mathrm{F}_{a b}\left(u^{s} v^{r}\right)\right]$ and $\overline{\mathrm{H}}_{a b}\left(u^{s} v^{r} \gamma\right)=$ $\left[\mathrm{F}_{a b}\left(u^{s} v^{r} \gamma\right), \mathrm{E}_{b a}\right]$. If $p=\widetilde{p} \gamma, \gamma \neq \mathrm{id}$, then $\widetilde{p}$ is a sum of monomials $u^{s} v^{r}$ of degree $N+1$, so we can also define $\mathrm{F}_{a b}(p)$ and $\overline{\mathrm{H}}_{a b}(p)$. 
Suppose that $\gamma \in \Gamma \backslash\{$ id $\}$ and let $u(\gamma), v(\gamma)$ be a basis of $U \cong \mathbb{C}^{2}$ consisting of eigenvectors of $\gamma$ (necessarily for nontrivial eigenvalues $\mu(\gamma), \nu(\gamma)$, respectively). The vectors $x(\gamma), y(\gamma)$ are defined similarly. For $s \geq 1$, we set $\widetilde{\mathrm{D}}\left(u(\gamma)^{s} v(\gamma)^{r} \gamma\right)=$ $\frac{1}{2(1-\mu(\gamma))} \sum_{i=1}^{n}\left[\overline{\mathrm{H}}_{i}\left(u(\gamma)^{s-1} v(\gamma)^{r} \gamma\right), \overline{\mathrm{H}}_{i}(u(\gamma))\right]$, whereas if $s=0$ and $r \geq 1$, we set $\widetilde{\mathrm{D}}\left(v(\gamma)^{r} \gamma\right)=\frac{1}{1-2(\nu(\gamma))} \sum_{i=1}^{n}\left[\overline{\mathrm{H}}_{i}\left(v(\gamma)^{r-1} \gamma\right), \overline{\mathrm{H}}_{i}(v(\gamma))\right]$. Let us assume that $s \geq 1$; then $\widetilde{\mathrm{D}}\left(u(\gamma)^{s} v(\gamma)^{r} \gamma\right)(\mathrm{h} \otimes \mathbf{v})$ equals

$$
\begin{aligned}
& \frac{1}{(1-\mu(\gamma))}\left(\sum_{k=1}^{l} \mathrm{~h}\left[x(\gamma)_{k}, x(\gamma)_{k}^{s} y(\gamma)_{k}^{r} \gamma_{k}\right] \otimes \mathbf{v}\right. \\
& \left.+\frac{1}{2} \sum_{i=1}^{n} \sum_{\substack{j, k=1 \\
j \neq k}}^{l} \mathrm{~h}\left[x(\gamma)_{k}, x(\gamma)_{j}^{s} y(\gamma)_{j}^{r} \gamma_{j}\right] \otimes H_{i}^{(k)} H_{i}^{(j)}(\mathbf{v})\right) \\
& =\sum_{k=1}^{l} \mathrm{~h} x(\gamma)_{k}^{s+1} y(\gamma)_{k}^{r} \gamma_{k} \otimes \mathbf{v} \\
& +\frac{\mu(\gamma)}{(1-\mu(\gamma))} \sum_{k=1}^{n} \sum_{d=0}^{r-1} h x(\gamma)_{k}^{s} y(\gamma)_{k}^{d}\left[x(\gamma)_{k}, y(\gamma)_{k}\right] y(\gamma)_{k}^{r-1-d} \gamma_{k} \otimes \mathbf{v} \\
& +\frac{1}{2(1-\mu(\gamma))} \sum_{i=1}^{n} \sum_{\substack{j, k=1 \\
j \neq k}}^{l} \sum_{d=0}^{s-1} \mathrm{~h} x(\gamma)_{j}^{d}\left[x(\gamma)_{k}, x(\gamma)_{j}\right] x(\gamma)_{j}^{s-1-d} y(\gamma)_{j}^{r} \gamma_{j} \otimes H_{i}^{(k)} H_{i}^{(j)}(\mathbf{v}) \\
& +\frac{1}{2(1-\mu(\gamma))} \sum_{i=1}^{n} \sum_{\substack{j, k=1 \\
j \neq k}}^{l} \sum_{d=0}^{r-1} \mathrm{~h} x(\gamma)_{j}^{s} y(\gamma)_{j}^{d}\left[x(\gamma)_{k}, y(\gamma)_{j}\right] y(\gamma)_{j}^{r-1-d} \gamma_{j} \otimes H_{i}^{(k)} H_{i}^{(j)}(\mathbf{v}) \\
& =\sum_{k=1}^{l} \mathrm{~h} x(\gamma)_{k}^{s+1} y(\gamma)_{k}^{r} \gamma_{k} \otimes \mathbf{v} \\
& +\frac{\mu(\gamma)}{(1-\mu(\gamma))} \sum_{k=1}^{n} \sum_{d=0}^{r-1} \mathrm{~h} x(\gamma)_{k}^{s} y(\gamma)_{k}^{d}\left(t+\frac{\kappa}{2} \sum_{\substack{j=1 \\
j \neq k}}^{l} \sum_{\tilde{\gamma} \in \Gamma} \sigma_{j k} \widetilde{\gamma}_{k} \widetilde{\gamma}_{j}^{-1}\right. \\
& \left.+\sum_{\tilde{\gamma} \in \Gamma \backslash\{\mathrm{id}\}} c_{\widetilde{\gamma}} \widetilde{\gamma}_{k}\right) y(\gamma)_{k}^{r-1-d} \gamma_{k} \otimes \mathbf{v} \\
& -\frac{\kappa}{4(1-\mu(\gamma))} \sum_{i=1}^{n} \sum_{\substack{j, k=1 \\
j \neq k}}^{l} \sum_{d=0}^{s-1} \sum_{\tilde{\gamma} \in \Gamma} \omega_{\widetilde{\gamma}}^{x(\gamma)} \mathrm{h} x(\gamma)_{j}^{d} \sigma_{j k} \widetilde{\gamma}_{k} \widetilde{\gamma}_{j}^{-1} x(\gamma)_{j}^{s-1-d} y(\gamma)_{j}^{r} \gamma_{j} \otimes H_{i}^{(k)} H_{i}^{(j)}(\mathbf{v}) \\
& -\frac{\kappa}{4(1-\mu(\gamma))} \sum_{i=1}^{n} \sum_{\substack{j, k=1 \\
j \neq k}}^{l} \sum_{d=0}^{r-1} \sum_{\tilde{\gamma} \in \Gamma} \omega_{\widetilde{\gamma}}^{x(\gamma), y(\gamma)} \mathrm{h} x(\gamma)_{j}^{s} y(\gamma)_{j}^{d} \sigma_{j k} \widetilde{\gamma}_{k} \widetilde{\gamma}_{j}^{-1} y(\gamma)_{j}^{r-1-d} \gamma_{j} \\
& \otimes H_{i}^{(k)} H_{i}^{(j)}(\mathbf{v}) \\
& =\sum_{k=1}^{l} \mathrm{~h} x(\gamma)_{k}^{s+1} y(\gamma)_{k}^{r} \gamma_{k} \otimes \mathbf{v}+\frac{\operatorname{tr} \mu(\gamma)}{(1-\mu(\gamma))} \sum_{k=1}^{n} \mathrm{~h} x(\gamma)_{k}^{s} y(\gamma)_{k}^{r-1} \gamma_{k} \otimes \mathbf{v} \\
& +\frac{\kappa \mu(\gamma)}{2(1-\mu(\gamma))} \sum_{\substack{j, k=1 \\
j \neq k}}^{l} \sum_{d=0}^{r-1} \sum_{\widetilde{\gamma} \in \Gamma} \mathrm{h} x(\gamma)_{k}^{s} y(\gamma)_{k}^{d} \widetilde{\gamma}(y(\gamma))_{j}^{r-1-d} \widetilde{\gamma}_{k}^{-1} \widetilde{\gamma}_{j} \gamma_{j} \sigma_{j k} \otimes \mathbf{v} \\
& +\frac{\mu(\gamma)}{(1-\mu(\gamma))} \sum_{k=1}^{l} \sum_{d=0}^{r-1} \sum_{\tilde{\gamma} \in \Gamma \backslash\{\mathrm{id}\}} c_{\widetilde{\gamma}} \mathrm{h} x(\gamma)_{k}^{s} y(\gamma)_{k}^{d} \widetilde{\gamma}(y(\gamma))_{k}^{r-1-d} \widetilde{\gamma}_{k} \gamma_{k} \otimes \mathbf{v}
\end{aligned}
$$




$$
\begin{aligned}
& -\frac{\kappa}{4(1-\mu(\gamma))} \sum_{i=1}^{n} \sum_{j \neq k} \sum_{\tilde{\gamma} \in \Gamma} \mathrm{h}\left(\sum_{d=0}^{s-1} \omega_{\widetilde{\gamma}}^{x(\gamma)} x(\gamma)_{j}^{d} \widetilde{\gamma}^{-1}(x(\gamma))_{k}^{s-1-d} \widetilde{\gamma}^{-1}(y(\gamma))_{k}^{r} \widetilde{\gamma}_{j} \widetilde{\gamma}_{k}^{-1} \gamma_{k}\right. \\
& \left.+\sum_{d=0}^{r-1} \omega_{\widetilde{\gamma}}^{x(\gamma), y(\gamma)} x(\gamma)_{j}^{s} y(\gamma)_{j}^{d} \widetilde{\gamma}^{-1}(y(\gamma))_{k}^{r-1-d} \widetilde{\gamma}_{j} \widetilde{\gamma}_{k}^{-1} \gamma_{k}\right) \\
& \otimes\left(2 E_{i i}^{(k)} E_{i i}^{(j)}-E_{i, i+1}^{(k)} E_{i+1, i}^{(j)}-E_{i+1, i}^{(k)} E_{i, i+1}^{(j)}\right)(\mathbf{v}) \\
& =\sum_{k=1}^{l} \mathrm{~h} x(\gamma)_{k}^{s+1} y(\gamma)_{k}^{r} \gamma_{k} \otimes \mathbf{v} \\
& +\frac{\operatorname{tr} \mu(\gamma)}{(1-\mu(\gamma))} \mathrm{I}\left(u(\gamma)^{s} v(\gamma)^{r-1} \gamma\right)(\mathbf{h} \otimes \mathbf{v})-\sum_{\tilde{\gamma} \in \Gamma} \sum_{a=1}^{n} r \mathrm{~F}_{a a}\left(u(\gamma)^{s} v(\gamma)^{r-1} \gamma\right)(\mathbf{h} \otimes \mathbf{v}) \\
& +\frac{\kappa \mu(\gamma)}{2(1-\mu(\gamma))} \sum_{d=0}^{r-1} \sum_{\tilde{\gamma} \in \Gamma} \sum_{a, b=1}^{n} \mathrm{~F}_{a b}\left(\widetilde{\gamma}(v(\gamma))^{r-1-d} \widetilde{\gamma} \gamma\right) \mathrm{F}_{b a}\left(u(\gamma)^{s} v(\gamma)^{d} \widetilde{\gamma}^{-1}\right)(\mathbf{h} \otimes \mathbf{v}) \\
& +\frac{\mu(\gamma)}{(1-\mu(\gamma))} \sum_{d=0}^{r-1} \sum_{\widetilde{\gamma} \in \Gamma \backslash\{\operatorname{id}\}} c_{\widetilde{\gamma}} \mathbf{I}\left(u(\gamma)^{s} v(\gamma)^{d} \widetilde{\gamma}(v(\gamma))^{r-1-d} \widetilde{\gamma} \gamma\right)(\mathbf{h} \otimes \mathbf{v}) \\
& -\frac{\kappa}{4(1-\mu(\gamma))} \sum_{i=1}^{n} \sum_{d=0}^{s-1} \sum_{\tilde{\gamma} \in \Gamma} \omega_{\widetilde{\gamma}}^{x(\gamma)}\left(2 \mathrm{~F}_{i i}\left(\widetilde{\gamma}^{-1}(u(\gamma))^{s-1-d} \widetilde{\gamma}^{-1}(v(\gamma))^{r} \widetilde{\gamma}^{-1} \gamma\right) \mathrm{F}_{i i}\left(u(\gamma)^{d} \widetilde{\gamma}\right)\right. \\
& -\mathrm{F}_{i+1, i}\left(\widetilde{\gamma}^{-1}(u(\gamma))^{s-1-d} \widetilde{\gamma}^{-1}(v(\gamma))^{r} \widetilde{\gamma}^{-1} \gamma\right) \mathrm{F}_{i, i+1}\left(u(\gamma)^{d} \widetilde{\gamma}\right) \\
& \left.-\mathrm{F}_{i, i+1}\left(\widetilde{\gamma}^{-1}(u(\gamma))^{s-1-d} \widetilde{\gamma}^{-1}(v(\gamma))^{r} \widetilde{\gamma}^{-1} \gamma\right) \mathrm{F}_{i+1, i}\left(u(\gamma)^{d} \widetilde{\gamma}\right)\right)(\mathbf{h} \otimes \mathbf{v}) \\
& -\frac{\kappa}{4(1-\mu(\gamma))} \sum_{i=1}^{n} \sum_{d=0}^{r-1} \sum_{\tilde{\gamma} \in \Gamma} \omega_{\widetilde{\gamma}}^{x(\gamma), y(\gamma)}\left(\mathrm{F}_{i i}\left(\widetilde{\gamma}^{-1}(v(\gamma))^{r-1-d} \widetilde{\gamma}^{-1} \gamma\right) \mathrm{F}_{i i}\left(u(\gamma)^{s} v(\gamma)^{d} \widetilde{\gamma}\right)\right. \\
& -\mathrm{F}_{i+1, i}\left(\widetilde{\gamma}(v(\gamma))^{r-1-d} \widetilde{\gamma}^{-1} \gamma\right) \mathrm{F}_{i, i+1}\left(u(\gamma)^{s} v(\gamma)^{d} \gamma\right) \\
& \left.-\mathrm{F}_{i, i+1}\left(\widetilde{\gamma}(v(\gamma))^{r-1-d} \widetilde{\gamma}^{-1} \gamma\right) \mathrm{F}_{i+1, i}\left(u(\gamma)^{s} v(\gamma)^{d} \gamma\right)\right)(\mathbf{h} \otimes \mathbf{v}) .
\end{aligned}
$$

Set $\mathrm{D}\left(u(\gamma)^{s} v(\gamma)^{r} \gamma\right)=\widetilde{\mathrm{D}}\left(u(\gamma)^{s} v(\gamma)^{r} \gamma\right)-(48)^{\prime}-\cdots-(\underline{56})^{\prime}$ where (48) denotes the expression on line (48) but without $(\mathbf{h} \otimes \mathbf{v})$. We define $\mathrm{F}_{i i}\left(u(\gamma)^{s} v(\gamma)^{r} \gamma\right)$ in the following way: $\mathrm{F}_{n n}\left(u(\gamma)^{s} v(\gamma)^{r} \gamma\right)=\frac{1}{n}\left(\mathrm{D}\left(u(\gamma)^{s} v(\gamma)^{r} \gamma\right)-\sum_{i=1}^{n-1} i \overline{\mathrm{H}}_{i}\left(u(\gamma)^{s} v(\gamma)^{r} \gamma\right)\right)$ and, recursively, $\mathrm{F}_{i i}\left(u(\gamma)^{s} v(\gamma)^{r} \gamma\right)=\overline{\mathrm{H}}_{i}\left(u(\gamma)^{s} v(\gamma)^{r} \gamma\right)+\mathrm{F}_{i+1, i+1}\left(u(\gamma)^{s} v(\gamma)^{r} \gamma\right)$ for $1 \leq i \leq n-1$. If $p=\widetilde{p} \gamma, \gamma \neq \mathrm{id}$, then $\widetilde{p}$ is a sum of monomials $u^{s} v^{r}$ of degree $N+1$, and $u^{s} v^{r}$ can be expressed uniquely as a sum of monomials in $u(\gamma), v(\gamma)$, so we can also define $\mathrm{F}_{i i}(p)$.

Finally, we should explain how to construct elements $\mathrm{F}_{i i}(p)$ when $p \in E[N+1]$. It is enough to consider the case when $p$ is a monomial $u(\gamma)^{s} v(\gamma) r$ on which some element $\gamma \neq$ id acts by the nonzero eigenvalue $\mu(\gamma)^{s-r}$. We have just seen how to define $\mathbf{F}_{i i}\left(u(\gamma)^{s} v(\gamma)^{r} \gamma\right)$, and we set $\mathbf{F}_{i i}\left(u(\gamma)^{s} v(\gamma)^{r}\right)=\left(1-\mu^{r-s}\right)^{-1}\left[\mathrm{~F}_{i i}\left(u(\gamma)^{s} v(\gamma)^{r} \gamma\right)\right.$, $\left.\mathrm{F}_{i i}\left(\gamma^{-1}\right)\right]$.

We have thus constructed elements $\mathrm{F}_{a b}(p) \forall p \in \mathbb{C}[u, v] \rtimes \Gamma, 1 \leq a, b \leq n$. Let

$$
\mathrm{B}=\left\{\mathrm{F}_{a b}\left(u^{s} v^{r} \gamma\right) \mid 1 \leq a, b \leq n, r, s \geq 0, \gamma \in \Gamma\right\} .
$$

Let us fix an $\leq$ order on B. We can assume that $\mathrm{F}_{a_{1} b_{1}}\left(u^{s_{1}} v^{r_{1}} \gamma_{1}\right)<\mathrm{F}_{a_{2} b_{2}}\left(u^{s_{2}} v^{r_{2}} \gamma_{2}\right)$ if $a_{1} \neq a_{2}$ and $a_{2}=b_{2}$.

Theorem 8.1. The canonical map $\mathrm{D}_{\beta=0, \mathbf{b}=\mathbf{0}}^{n} \longrightarrow g r_{F}\left(\mathrm{D}_{\beta, \mathbf{b}}^{n}\right)$ is an isomorphism.

Proof. We follow the same ideas as in Gu2]. We will prove that, when $\beta+\lambda-\frac{n \lambda|\Gamma|}{4} \neq$ 0 , the set of ordered monomials in the elements of $B$ is a vector space basis of $D_{\beta, \mathbf{b}}^{n}$. 
Suppose that we have a nontrivial relation of the type

$$
\sum_{d \in S_{1}} \sum_{(A, B, R, S, \underline{\gamma}, E) \in S_{2}(d)} c(d, A, B, R, S, \underline{\gamma}, E) \mathbf{M}(d, A, B, R, S, \underline{\gamma}, E)=0,
$$

where $S_{1}, S_{2}(d)$ are finite sets, $S_{1} \subset \mathbb{Z}_{\geq 0}, S_{2}(d) \subset[1, n]^{\times d} \times[1, n]^{\times d} \times\left(\mathbb{Z}_{\geq 0}\right)^{\times d} \times$ $\left(\mathbb{Z}_{\geq 0}\right)^{\times d} \times \Gamma^{\times d} \times\left(\mathbb{Z}_{>0}\right)^{\times d}, A=\left(a_{1}, \ldots, a_{d}\right), B=\left(b_{1}, \ldots, b_{d}\right), R=\left(r_{1}, \ldots, r_{d}\right), S=$ $\left(s_{1}, \ldots, s_{d}\right), \underline{\gamma}=\left(\gamma^{1}, \ldots, \gamma^{d}\right), E=\left(e_{1}, \ldots, e_{d}\right),[1, n]=\{1, \ldots, n\}$ and

$$
\mathbf{M}(d, A, B, R, S, \underline{\gamma}, E)=\mathrm{F}_{a_{1} b_{1}}\left(u^{s_{1}} v^{r_{1}} \gamma^{1}\right)^{e_{1}} \cdots \mathrm{F}_{a_{d} b_{d}}\left(u^{s_{d}} v^{r_{d}} \gamma^{d}\right)^{e_{d}}
$$

is an ordered monomial in the elements of B. (In particular, $\mathrm{F}_{a_{i} b_{i}}\left(u^{s_{i}} v^{r_{i}} \gamma^{i}\right) \neq$ $\mathrm{F}_{a_{j} b_{j}}\left(u^{s_{j}} v^{r_{j}} \gamma^{j}\right)$ if $i \neq j$.)

Let us choose a specific $(\check{d}, \check{A}, \check{B}, \check{R}, \check{S}, \underline{\underline{\underline{x}}}, \check{E})$ such that

(1) $\sum_{g=1}^{d}\left(r_{g}+s_{g}\right) e_{g}$ has maximum value for which $c(d, A, B, R, S, \underline{\gamma}, E) \neq 0$;

(2) among these, it has maximum value for $\sum_{g=1}^{d}\left(1-\delta_{a_{g} b_{g}}\right) e_{g}$;

(3) among these, it has maximum value for $\sum_{g=1}^{d} \delta_{a_{g} b_{g}} e_{g}$.

This choice may not be unique. Set $\check{M}=\mathbf{M}(\check{d}, \check{A}, \check{B}, \check{R}, \check{S}, \check{\gamma}, \check{E})$. Set $\delta=$ $\sum_{g=1}^{d} e_{g}$. Now suppose that $l, l_{1}, \ldots, l_{n} \in \mathbb{Z}_{\geq 0}$ are such that $l_{1}+\cdots+l_{n}=l-\delta$. We let $\mathbf{v}=v^{1} \otimes \cdots \otimes v^{l}, \widehat{\mathbf{v}}=\widehat{v}^{1} \otimes \cdots \otimes \widehat{v}^{l}$ be the following elements of $\left(\mathbb{C}^{n}\right)^{\otimes l}$ for $l \geq \delta$ : for $e_{1}+\cdots+e_{j-1}+1 \leq g \leq e_{1}+\cdots+e_{j}$, set $v^{g}=v_{b_{j}}, \widehat{v}^{g}=v_{a_{j}}, m_{g}=x_{g}^{s_{i}} y_{g}^{r_{i}}$, $\widetilde{\gamma}_{g}=\gamma_{g}^{i} \in \Gamma^{\times l}$, and set $v^{g}=\widehat{v}^{g}=v_{i}$ if $\delta+l_{1}+\cdots+l_{i-1}+1 \leq g \leq \delta+l_{1}+\cdots+l_{i}$.

Because of our assumption that equality (57) holds,

$$
\sum_{d \in S_{1}} \sum_{(A, B, R, S, \underline{\gamma}, E) \in S_{2}(d)} c(d, A, B, R, S, \underline{\gamma}, E) \mathbf{M}(d, A, B, R, S, \underline{\gamma}, E)(1 \otimes \mathbf{v})=0 .
$$

Consider the vector space basis of $\mathrm{H}_{t, \mathbf{c}}\left(\Gamma_{l}\right) \otimes_{\mathbb{C}\left[S_{l}\right]}\left(\mathbb{C}^{n}\right)^{\otimes l}$ (with $t, \mathbf{c}, \beta, \mathbf{b}$ as in section 6. so that this space is a module for $\mathrm{D}_{\beta, \mathbf{b}}^{n}$ ) given by the monomials $x_{l}^{q_{l}^{x}} y_{l}^{q_{l}^{y}} \cdots x_{1}^{q_{1}^{x}} y_{1}^{q_{1}^{y}} \gamma$ $\otimes \overline{\mathbf{v}}$, where $q_{i}^{x}, q_{i}^{y} \in \mathbb{Z}_{\geq 0}, \gamma \in \Gamma^{\times l}, \overline{\mathbf{v}}=\bar{v}^{1} \otimes \cdots \otimes \bar{v}^{l}$ with $\bar{v}^{g} \in\left\{v_{1}, \ldots, v_{n}\right\}$. We can decompose the left-hand side of (58) as a sum of vectors in that basis and do the same for $\check{\mathbf{M}}(1 \otimes \mathbf{v})$. The coefficient of $m_{\delta} m_{\delta-1} \cdots m_{1} \widetilde{\gamma}_{\delta} \cdots \widetilde{\gamma}_{1} \otimes \widehat{\mathbf{v}}$ in $\check{\mathbf{M}}(1 \otimes \mathbf{v})$ is equal to $\tilde{c} c(\breve{d}, \check{A}, \breve{B}, \check{R}, \check{S}, \check{\underline{\gamma}}, \check{E})$ for some $\tilde{c} \neq 0$ which depends on the multiplicities $e_{i}, l_{j}$. Furthermore, the only other monomials in the left-hand side of (57) which can produce a nonzero multiple of $m_{\delta} m_{\delta-1} \cdots m_{1} \gamma_{\delta} \cdots \gamma_{1} \otimes \widehat{\mathbf{v}}$ when applied to $1 \otimes \mathbf{v}$ differ from $\check{\mathbf{M}}$ only by the value of $e_{g}$ for $g$ such that $a_{g}=b_{g}, m_{g}=1, \widetilde{\gamma}_{g}=$ id. Because of our assumption on the order on B, these elements always appear at the end of each monomial in (57). Therefore, the coefficient of $m_{\delta} m_{\delta-1} \cdots m_{1} \gamma_{\gamma} \cdots \gamma_{1} \otimes \widehat{\mathbf{v}}$ in the left-hand side of (58) can be viewed as a polynomial in $l_{1}, \ldots, l_{n}$. Since this polynomial vanishes for infinitely many values of these variables, which can be given arbitrarily large independent values, it must vanish identically, so its coefficients are zero and $c(\check{d}, \breve{A}, \check{B}, \check{R}, \breve{S}, \check{\gamma}, \breve{E})=0$. Repeating this argument, we conclude that all the coefficients $c(d, A, B, R, S, \underline{\gamma}, E)$ in (57) equal zero. This completes the proof of the linear independance when $\beta+\lambda-\frac{\lambda n|\Gamma|}{4} \neq 0$. This means that the map $\mathrm{D}_{\beta=0, \mathbf{b}=\mathbf{0}}^{n} \rightarrow \operatorname{gr}_{F}\left(\mathrm{D}_{\beta, \mathbf{b}}^{n}\right)$ is an isomorphism if $\beta+\lambda-\frac{\lambda n|\Gamma|}{4} \neq 0$. By uppersemicontinuity, it must be an isomorphism for any $\beta, \mathbf{b}$. 
Let $\Phi_{l}$ be the algebra homomorphism $\mathrm{D}_{\beta, \mathbf{b}}^{n} \longrightarrow \operatorname{End}_{\mathbb{C}}\left(\mathrm{V}^{l}\right)$ coming from the $\mathrm{D}_{\beta, \mathbf{b}^{-}}^{n}$ module structure on $\mathrm{H}_{t, \mathbf{c}}\left(\Gamma_{l}\right) \otimes_{\mathbb{C}\left[S_{l}\right]}\left(\mathbb{C}^{n}\right)^{\otimes l}$.

Corollary 8.1 (of the proof of Theorem 8.1). Suppose that $\beta+\lambda-\frac{\lambda n|\Gamma|}{4} \neq 0$. Given $M \in \mathrm{D}_{\beta, \mathbf{b}}^{n}$, there exists an $l \gg 0$ such that $\Phi_{l}(M)$ is not identically zero.

\section{Cyclotomic case}

It is possible to generalize most of the results of Gu1 and Gu2 to the case when $\Gamma$ is a cyclic group of order $d$. In order to do this, we first have to consider a family of graded Hecke algebras for the complex reflection groups $S_{l}$ 々 $\mathbb{Z} / d \mathbb{Z}$ which were first introduced in RaSh and studied when $d=2$ in [De1 and, in general, in De2. We will then prove an equivalence of Schur-Weyl type between a localization of a rational Cherednik algebra and an affine Yangian, generalizing the work in Gu1]. Afterwards, we will explain how to realize the $\mathbb{Z} / d \mathbb{Z}$-deformed double current algebra $D_{\beta, \mathbf{b}}^{n}$ as a subalgebra of this affine Yangian.

9.1. Graded Hecke algebras for $S_{l} \prec \mathbb{Z} / d \mathbb{Z}$. Fix $l, d \geq 1$. Set $\Gamma=\mathbb{Z} / d \mathbb{Z}$ and let $\Gamma_{l}$ be the complex reflection group $G(l, 1, d)$, which is the wreath product $\Gamma_{l}=$ $S_{l} \prec \mathbb{Z} / d \mathbb{Z}=\mathbb{Z} / d \mathbb{Z}^{\times l} \rtimes S_{l}$. It is generated by reflections $\sigma_{i, i+1} \in S_{l}, 1 \leq i \leq l-1$, and by pseudo-reflections $\xi_{i}$ of order $d$, so $\left\langle\xi_{i}^{j}\right\rangle_{j=0}^{d-1} \cong \mathbb{Z} / d \mathbb{Z}, 1 \leq i \leq l$. Let $\mathfrak{h} \cong \mathbb{C}^{l}$ be its reflection representation and choose dual bases $\left\{y_{1}, \ldots, y_{l}\right\},\left\{x_{1}, \ldots, x_{l}\right\}$ of $\mathfrak{h}$ and $\mathfrak{h}^{*}$. Fix a primitive $d^{\text {th }}$-root of unity $\zeta$ so that $\xi_{i}\left(x_{i}\right)=\zeta x_{i}, \xi_{i}\left(y_{i}\right)=\zeta^{-1} y_{i}$. Let $\mathbf{e}_{i}, i=0, \ldots, d-1$, be the primitive idempotents of $\mathbb{Z} / d \mathbb{Z}$, so $\mathbf{e}_{i}=\frac{1}{d} \sum_{j=0}^{d-1} \zeta^{-j i} \xi^{j}$, and let $\mathbf{e}_{i, k}$ be the idempotent of $\Gamma_{l}$ corresponding to $\mathbf{e}_{i}$ in the $k^{\text {th }}$ copy of $\Gamma$ in $\Gamma_{l}$, so $\mathbf{e}_{i, k}=\frac{1}{d} \sum_{j=0}^{d-1} \zeta^{-j i} \xi_{k}^{j}$.

Definition 9.1 ( $\mathrm{RaSh})$. Let $\kappa \in \mathbb{C}$. We define the degenerate affine Hecke algebra of $\Gamma_{l}$ to be the algebra $\mathrm{H}_{\kappa}\left(\Gamma_{l}\right)$ generated by $\Gamma_{l}$ and by the pairwise commuting elements $\left\{u_{i}\right\}_{i=1}^{l}$ which obey the following relations:

$$
\begin{gathered}
\xi_{j} u_{i}=u_{i} \xi_{j} \text { for any } 1 \leq i, j \leq l, \quad \sigma_{i} u_{j}=u_{j} \sigma_{i} \text { if } j \neq i, i+1, \\
\sigma_{i} u_{i+1}=u_{i} \sigma_{i}+\kappa \sum_{k=0}^{d-1} \xi_{i}^{-k} \xi_{i+1}^{k} \text { for } 1 \leq i \leq l-1 .
\end{gathered}
$$

Remark 9.1. Note that, when $r=2, \Gamma_{l}$ is the Weyl group of type B (or C), but the algebra $\mathrm{H}_{\kappa}\left(\Gamma_{l}\right)$ is not isomorphic to the degenerate Hecke algebra of this type as defined originally in [Dr1], [Lu]. If $\kappa \neq 0$, then $\mathrm{H}_{\kappa}\left(\Gamma_{l}\right) \cong \mathrm{H}_{\kappa=1}\left(\Gamma_{l}\right)$.

Proposition 9.1. The elements of the form $p\left(u_{1}, \ldots, u_{l}\right) w$ constitute a basis of $\mathrm{H}_{\kappa}\left(\Gamma_{l}\right)$, where $w \in \Gamma_{l}$ and $p\left(u_{1}, \ldots, u_{l}\right)$ is a monomial in the variables $u_{1}, \ldots, u_{l}$.

The proof of Proposition 9.1 given below uses the PBW property of a subalgebra of $\mathrm{H}_{t, c}\left(\Gamma_{l}\right)$; see Proposition 9.2. It will be convenient to rewrite Definition 2.1] in a slightly different form. 
Definition 9.2 (DuOp, EtGi $)$. The rational Cherednik algebra $\mathrm{H}_{t, \mathbf{c}}\left(\Gamma_{l}\right)$ of $\Gamma_{l}$ with (multi-)parameters $t \in \mathbb{C}, \mathbf{c}=\left(\kappa, c_{1}, \ldots, c_{d-1}\right) \in \mathbb{C}^{d}$ is the algebra generated by $\mathbb{C}[\mathfrak{h}], \mathbb{C}\left[\mathfrak{h}^{*}\right]$ and $\Gamma_{l}$ subject to the following relations:

$$
\begin{gathered}
\sigma \cdot x \cdot \sigma^{-1}=\sigma(x), \quad \sigma \cdot y \cdot \sigma^{-1}=\sigma(y) \quad \text { for } x \in \mathfrak{h}^{*}, y \in \mathfrak{h}, \sigma \in \Gamma_{l}, \\
{\left[y_{j}, x_{i}\right]=y_{j} x_{i}-x_{i} y_{j}=-\kappa \sum_{k=0}^{d-1} \zeta^{k} \xi_{i}^{-k} \sigma_{i j} \xi_{i}^{k} \text { if } i \neq j,} \\
{\left[y_{i}, x_{i}\right]=y_{i} x_{i}-x_{i} y_{i}=t+\kappa \sum_{\substack{j=1 \\
j \neq i}}^{l} \sum_{k=0}^{d-1} \xi_{i}^{-k} \sigma_{i j} \xi_{i}^{k}+\sum_{k=1}^{d-1} d c_{k}\left(\mathbf{e}_{k, i}-\mathbf{e}_{k-1, i}\right) .}
\end{gathered}
$$

We denote by $\mathcal{Y}_{i}, \mathcal{U}_{i}$ the following elements of $\mathrm{H}_{t, \mathbf{c}}\left(\Gamma_{l}\right)$ (see DuOp):

$$
\begin{aligned}
\mathcal{Y}_{i}= & \frac{1}{2}\left(x_{i} y_{i}+y_{i} x_{i}\right)=\frac{t}{2}+x_{i} y_{i}+\frac{\kappa}{2} \sum_{\substack{j=1 \\
j \neq i}}^{l} \sum_{k=0}^{d-1} \xi_{i}^{-k} \sigma_{i j} \xi_{i}^{k} \\
& +\frac{1}{2} \sum_{k=1}^{d-1} d c_{k}\left(\mathbf{e}_{k, i}-\mathbf{e}_{k-1, i}\right), \\
\mathcal{U}_{i}= & \frac{t}{2}+x_{i} y_{i}+\kappa \sum_{1 \leq j<i} \sum_{k=0}^{d-1} \xi_{i}^{-k} \sigma_{i j} \xi_{i}^{k}+\sum_{k=1}^{d-1} d c_{k} \mathbf{e}_{k, i} \\
= & \mathcal{Y}_{i}-\frac{\kappa}{2} \sum_{\substack{j=1 \\
j \neq i}}^{l} \operatorname{sign}(j-i) \sum_{k=0}^{d-1} \xi_{i}^{-k} \sigma_{i j} \xi_{i}^{k}+\frac{1}{2} \sum_{k=1}^{d-1} d c_{k}\left(\mathbf{e}_{k, i}+\mathbf{e}_{k-1, i}\right) .
\end{aligned}
$$

In the computations below, the following formulas will be useful:

(61) $\left[\mathcal{Y}_{i}, x_{i}\right]=t x_{i}+\frac{\kappa}{2} \sum_{\substack{j=1 \\ j \neq i}}^{l} \sum_{k=0}^{d-1}\left(x_{i}+\zeta^{k} x_{j}\right) \xi_{i}^{-k} \sigma_{i j} \xi_{i}^{k}+\frac{1}{2} \sum_{k=1}^{d-1} d c_{k}\left(\mathbf{e}_{k+1, i}-\mathbf{e}_{k-1, i}\right) x_{i}$.

We need to obtain an expression for $\left[\mathcal{Y}_{i}, \mathcal{Y}_{j}\right]$ for $i \neq j$ which will be useful later, so we compute: [1]

$$
\text { For } i \neq j,\left[\mathcal{Y}_{j}, x_{i}\right]=-\frac{\kappa}{2} \sum_{k=0}^{d-1}\left(\zeta^{k} x_{j}+x_{i}\right) \xi_{i}^{-k} \sigma_{i j} \xi_{i}^{k},
$$

$$
\text { (64) }+\frac{d \kappa}{4} \sum_{\substack{a=1 \\ k=0}}^{d-1}\left(\left[c_{a}\left(\mathbf{e}_{a, i}-\mathbf{e}_{a-1, i}\right), \sum_{\substack{b=1 \\ b \neq j}}^{l} \xi_{j}^{-k} \sigma_{b j} \xi_{j}^{k}\right]+\left[\sum_{\substack{b=1 \\ b \neq i}}^{l} \xi_{i}^{-k} \sigma_{b i} \xi_{i}^{k}, c_{a}\left(\mathbf{e}_{a, j}-\mathbf{e}_{a-1, j}\right)\right]\right) \text {. }
$$


We compute each of the sums in (62), (63) and (64) separately.

$$
\begin{aligned}
& \text { (62) }=-\kappa x_{i} \sum_{k=0}^{d-1} \zeta^{k} \xi_{j}^{-k} \sigma_{i j} \xi_{j}^{k} y_{j}+\kappa x_{j} \sum_{k=0}^{d-1} \zeta^{k} \xi_{i}^{-k} \sigma_{i j} \xi_{i}^{k} y_{i} \\
& +\frac{\kappa}{2} \sum_{k=0}^{d-1}\left[x_{i} y_{i}, \xi_{j}^{-k} \sigma_{i j} \xi_{j}^{k}\right]+\frac{\kappa}{2} \sum_{k=0}^{d-1}\left[\xi_{i}^{-k} \sigma_{i j} \xi_{i}^{k}, x_{j} y_{j}\right]=0 \\
& \text { (63) }=\frac{\kappa^{2}}{4} \sum_{\substack{b=1 \\
b \neq i, j}}^{l} \sum_{k, m=0}^{d-1}\left[\xi_{i}^{-m} \sigma_{b i} \xi_{i}^{m}, \xi_{j}^{-k} \sigma_{b j} \xi_{j}^{k}\right]+\frac{\kappa^{2}}{4} \sum_{\substack{b=1 \\
b \neq i, j}}^{l} \sum_{k, m=0}^{d-1}\left[\xi_{i}^{-m} \sigma_{b i} \xi_{i}^{m}, \xi_{j}^{-k} \sigma_{i j} \xi_{j}^{k}\right] \\
& +\frac{\kappa^{2}}{4} \sum_{\substack{b=1 \\
b \neq i, j}}^{l} \sum_{k, m=0}^{d-1}\left[\xi_{i}^{-m} \sigma_{i j} \xi_{i}^{m}, \xi_{j}^{-k} \sigma_{b j} \xi_{j}^{k}\right] \\
& =\frac{\kappa^{2}}{4} \sum_{\substack{b=1 \\
b \neq i, j}}^{l} \sum_{k, m=0}^{d-1}\left(\xi_{i}^{-m+k} \xi_{b}^{m} \xi_{j}^{-k} \sigma_{b i} \sigma_{b j}-\xi_{j}^{-k} \xi_{b}^{k+m} \xi_{i}^{-m} \sigma_{b j} \sigma_{b i}\right. \\
& +\xi_{i}^{-m} \xi_{b}^{m+k} \xi_{j}^{-k} \sigma_{b j} \sigma_{b i}-\xi_{j}^{-k-m} \xi_{i}^{k} \xi_{b}^{m} \sigma_{b i} \sigma_{b j} \\
& \left.+\xi_{i}^{-m-k} \xi_{j}^{m} \xi_{b}^{k} \sigma_{b j} \sigma_{b i}-\xi_{j}^{-k} \xi_{b}^{k+m} \xi_{i}^{-m} \sigma_{b i} \sigma_{b j}\right) \\
& =\frac{\kappa^{2}}{4} \sum_{\substack{b=1 \\
b \neq i, j}}^{l} \sum_{k, m=0}^{d-1} \xi_{i}^{-m-k} \xi_{j}^{m} \xi_{b}^{k}\left[\sigma_{b j}, \sigma_{b i}\right] \\
& \left(\underline{64)}=\frac{\kappa}{4} \sum_{a=1}^{d-1} d c_{a} \sum_{k=0}^{d-1}\left(\left[\left(\mathbf{e}_{a, i}-\mathbf{e}_{a-1, i}\right), \xi_{j}^{-k} \sigma_{i j} \xi_{j}^{k}\right]+\left[\xi_{i}^{-k} \sigma_{i j} \xi_{i}^{k},\left(\mathbf{e}_{a, j}-\mathbf{e}_{a-1, j}\right)\right]\right)=0 .\right.
\end{aligned}
$$

Therefore,

$$
\left[\mathcal{Y}_{i}, \mathcal{Y}_{j}\right]=\frac{\kappa^{2}}{4} \sum_{\substack{b=1 \\ b \neq i, j}}^{l} \sum_{k, m=0}^{d-1} \xi_{i}^{-m-k} \xi_{j}^{m} \xi_{b}^{k}\left[\sigma_{b j}, \sigma_{b i}\right]
$$

Let $\widetilde{\mathrm{H}}_{t, \mathbf{c}}\left(\Gamma_{l}\right)$ be the subalgebra of $\mathrm{H}_{t, \mathbf{c}}\left(\Gamma_{l}\right)$ generated by $\Gamma_{l}$ and $\mathcal{U}_{i}, 1 \leq i \leq l$.

Proposition $9.2(\mathrm{DuOp})$ ). The elements $\mathcal{U}_{1}, \ldots, \mathcal{U}_{l}$ commute pairwise. Moreover, the elements of the form $p\left(\mathcal{U}_{1}, \ldots, \mathcal{U}_{l}\right) w$, where $p\left(\mathcal{U}_{1}, \ldots, \mathcal{U}_{l}\right)$ is a monomial and $w \in \Gamma_{l}$, form a vector space basis of the algebra $\widetilde{\mathrm{H}}_{t, \mathbf{c}}\left(\Gamma_{l}\right)$. 
Proof. In view of equality (59) above, it is enough to show that

$$
\left[\mathcal{Y}_{i}, \mathcal{Y}_{j}\right]=-\frac{\kappa^{2}}{4}\left[\sum_{\substack{b=1 \\ b \neq i}}^{l} \operatorname{sign}(b-i) \sum_{k=0}^{d-1} \xi_{i}^{-k} \sigma_{b i} \xi_{i}^{k}, \sum_{\substack{e=1 \\ e \neq j}}^{l} \operatorname{sign}(e-j) \sum_{m=0}^{d-1} \xi_{j}^{-m} \sigma_{e j} \xi_{j}^{m}\right]
$$

Let us assume that $i<j$. The right-hand side is equal to

$$
\begin{aligned}
& -\frac{\kappa^{2}}{4} \operatorname{sign}(b-j) \sum_{k, m=0}^{d-1} \sum_{\substack{b=1 \\
b \neq i, j}}^{l}\left[\xi_{i}^{-k} \sigma_{j i} \xi_{i}^{k}, \xi_{j}^{-m} \sigma_{b j} \xi_{j}^{m}\right] \\
& +\frac{\kappa^{2}}{4} \operatorname{sign}(b-i) \sum_{k, m=0}^{d-1} \sum_{\substack{b=1 \\
b \neq i, j}}^{l}\left[\xi_{i}^{-k} \sigma_{b i} \xi_{i}^{k}, \xi_{j}^{-m} \sigma_{i j} \xi_{j}^{m}\right] \\
& -\frac{\kappa^{2}}{4} \operatorname{sign}((b-i)(b-j)) \sum_{k, m=0}^{d-1} \sum_{\substack{b=1 \\
b \neq i, j}}^{l}\left[\xi_{i}^{-k} \sigma_{b i} \xi_{i}^{k}, \xi_{j}^{-m} \sigma_{b j} \xi_{j}^{m}\right] \\
& =\frac{\kappa^{2}}{4} \sum_{k, m=0}^{d-1} \sum_{b<i}\left(\xi_{i}^{-k-m} \xi_{j}^{k} \xi_{b}^{m} \sigma_{j i} \sigma_{b j}-\xi_{j}^{-m} \xi_{b}^{m+k} \xi_{i}^{-k} \sigma_{b j} \sigma_{i j}-\xi_{i}^{-k} \xi_{b}^{m+k} \xi_{j}^{-m} \sigma_{b i} \sigma_{i j}\right. \\
& \left.+\xi_{j}^{-m-k} \xi_{i}^{m} \xi_{b}^{k} \sigma_{i j} \sigma_{b i}-\xi_{i}^{-k+m} \xi_{j}^{-m} \xi_{b}^{k} \sigma_{b i} \sigma_{b j}+\xi_{j}^{-m+k} \xi_{b}^{m} \xi_{i}^{-k} \sigma_{b j} \sigma_{b i}\right) \\
& +\frac{\kappa^{2}}{4} \sum_{k, m=0}^{d-1} \sum_{b>j}\left(-\xi_{i}^{-k-m} \xi_{j}^{k} \xi_{b}^{m} \sigma_{j i} \sigma_{b j}+\xi_{j}^{-m} \xi_{b}^{m+k} \xi_{i}^{-k} \sigma_{b j} \sigma_{i j}+\xi_{i}^{-k} \xi_{b}^{m+k} \xi_{j}^{-m} \sigma_{b i} \sigma_{i j}\right. \\
& \left.-\xi_{j}^{-m-k} \xi_{i}^{m} \xi_{b}^{k} \sigma_{i j} \sigma_{b i}-\xi_{i}^{-k+m} \xi_{j}^{-m} \xi_{b}^{k} \sigma_{b i} \sigma_{b j}+\xi_{j}^{-m+k} \xi_{b}^{m} \xi_{i}^{-k} \sigma_{b j} \sigma_{b i}\right) \\
& +\frac{\kappa^{2}}{4} \sum_{k, m=0}^{d-1} \sum_{i<b<j}\left(\xi_{i}^{-k-m} \xi_{j}^{k} \xi_{b}^{m} \sigma_{j i} \sigma_{b j}-\xi_{j}^{-m} \xi_{b}^{m+k} \xi_{i}^{-k} \sigma_{b j} \sigma_{i j}+\xi_{i}^{-k} \xi_{b}^{m+k} \xi_{j}^{-m} \sigma_{b i} \sigma_{i j}\right. \\
& \left.-\xi_{j}^{-m-k} \xi_{i}^{m} \xi_{b}^{k} \sigma_{i j} \sigma_{b i}+\xi_{i}^{-k+m} \xi_{j}^{-m} \xi_{b}^{k} \sigma_{b i} \sigma_{b j}-\xi_{j}^{-m+k} \xi_{b}^{m} \xi_{i}^{-k} \sigma_{b j} \sigma_{b i}\right) \\
& =\frac{\kappa^{2}}{4} \sum_{\substack{b=1 \\
b \neq i, j}}^{l} \sum_{k, m=0}^{d-1} \xi_{i}^{-m-k} \xi_{j}^{m} \xi_{b}^{k}\left[\sigma_{b j}, \sigma_{b i}\right]=\left[\mathcal{Y}_{i}, \mathcal{Y}_{j}\right] \text {, }
\end{aligned}
$$

where the last equality is (65) below.

The second part of the proposition follows immediately from the PBW theorem for $\mathrm{H}_{t, \mathbf{c}}\left(\Gamma_{l}\right)$; see, e.g., [EtGi]. 
Proof of Proposition 9.1. It follows from the definition of $\mathrm{H}_{\kappa}\left(\Gamma_{l}\right)$ that the elements $p\left(u_{1}, \ldots, u_{n}\right) w$ span $\mathrm{H}_{\kappa}\left(\Gamma_{l}\right)$ as a vector space. We have to show that they are linearly independent. The operators $\mathcal{U}_{i}$ satisfy the same relations as the elements $u_{i}$ in $\mathrm{H}_{\kappa}\left(\Gamma_{l}\right)$ (see $\left.\mathrm{DuOp}\right)$. For instance,

$$
\begin{aligned}
\sigma_{i} \mathcal{U}_{i+1} & =\frac{t}{2} \sigma_{i}+\sigma_{i} x_{i+1} y_{i+1}+\kappa \sum_{1 \leq j<i+1} \sum_{k=0}^{d-1} \sigma_{i} \xi_{i+1}^{-k} \sigma_{i+1, j} \xi_{i+1}^{k}+\sigma_{i} \sum_{k=1}^{d-1} d c_{k} \mathbf{e}_{k, i+1} \\
& =\frac{t}{2} \sigma_{i}+x_{i} y_{i} \sigma_{i}+\kappa \sum_{j<i} \sum_{k=0}^{d-1} \xi_{i}^{-k} \sigma_{i j} \xi_{i}^{k} \sigma_{i}+\kappa \sum_{k=0}^{d-1} \xi_{i}^{-k} \xi_{i+1}^{k}+\sum_{k=1}^{d-1} d c_{k} \mathbf{e}_{a, i} \sigma_{i} \\
& =\mathcal{U}_{i} \sigma_{i}+\kappa \sum_{k=0}^{d-1} \xi_{i}^{-k} \xi_{i+1}^{k} .
\end{aligned}
$$

This shows that we have an algebra epimorphism $\mathrm{H}_{\kappa}\left(\Gamma_{l}\right) \longrightarrow \widetilde{\mathrm{H}}_{t, \mathbf{c}}\left(\Gamma_{l}\right)$, which must also be injective because of the PBW property of $\widetilde{H}_{t, \mathbf{c}}\left(\Gamma_{l}\right)$.

Corollary 9.1 (Corollary of the proof of Proposition 9.1). The algebras $\mathrm{H}_{\kappa}\left(\Gamma_{l}\right)$ and $\widetilde{\mathrm{H}}_{t, \mathbf{c}}\left(\Gamma_{l}\right)$ are isomorphic.

From Corollary 9.1 and the computations before Proposition 9.2, we deduce the following proposition.

Proposition 9.3. The algebra $\mathrm{H}_{\kappa}\left(\Gamma_{l}\right)$ can also be defined as the algebra generated by elements $\mathrm{y}_{i}, 1 \leq i \leq l$, and $\gamma \in \Gamma_{l}$ satisfying the following relations:

$$
\begin{array}{r}
\text { If } i \neq j,\left[\mathrm{y}_{i}, \mathrm{y}_{j}\right]=\frac{\kappa^{2}}{4} \sum_{\substack{b=1 \\
b \neq i, j}}^{l} \sum_{k, m=0}^{d-1} \xi_{i}^{-m-k} \xi_{j}^{m} \xi_{b}^{k}\left[\sigma_{b j}, \sigma_{b i}\right], \sigma \mathrm{y}_{i} \sigma^{-1}=\mathrm{y}_{\sigma(i)}, \\
\xi_{a} \mathrm{y}_{i}=\mathrm{y}_{i} \xi_{a}, \sigma \in S_{l}, 1 \leq a \leq l .
\end{array}
$$

Setting $\mathrm{y}_{i, k}=\mathrm{y}_{i} \mathbf{e}_{k, i}, 1 \leq i \leq l, 0 \leq k \leq d-1$, we see that $\mathrm{H}_{\kappa}\left(\Gamma_{l}\right)$ can also be defined in the following way, which will be useful later.

Proposition 9.4. The algebra $\mathrm{H}_{\kappa}\left(\Gamma_{l}\right)$ is isomorphic to the algebra generated by the elements $\mathrm{y}_{i, k}, 1 \leq i \leq l, 0 \leq k \leq d-1$, the idempotents $\mathbf{e}_{k, i} \in \mathbb{C}\left[\Gamma_{l}\right]$ and $\sigma \in S_{l}$ which satisfy the following relations:

$$
\begin{gathered}
{\left[\mathrm{y}_{i, k_{1}}, \mathrm{y}_{j, k_{2}}\right]=0 \text { if } k_{1} \neq k_{2}, \quad \mathbf{e}_{k_{1}, i} \mathrm{y}_{i, k_{2}}=\delta_{k_{1} k_{2}} \mathrm{y}_{i, k_{2}}=\mathrm{y}_{i, k_{2}} \mathbf{e}_{k_{1}, i},} \\
\quad \mathbf{e}_{k_{1}, j} \mathrm{y}_{i, k_{2}}=\mathrm{y}_{i, k_{2}} \mathbf{e}_{k_{1}, j} \text { if } i \neq j, \\
\sigma \mathrm{y}_{i, k}=\mathrm{y}_{\sigma(i), k} \sigma, \quad\left[\mathrm{y}_{i, k}, \mathrm{y}_{j, k}\right]=\frac{d^{2} \kappa^{2}}{4} \sum_{\substack{b=1 \\
b \neq i, j}}^{l} \mathbf{e}_{k, i} \mathbf{e}_{k, j} \mathbf{e}_{k, b}\left[\sigma_{b i}, \sigma_{b j}\right] \text { if } i \neq j .
\end{gathered}
$$


For each Weyl group, I. Cherednik has introduced the notion of double affine Hecke algebras (DAHA) Ch1]. These algebras afford two types of limit versions called degenerate (trigonometric) DAHA's and rational DAHA's. These rational Cherednik algebras can be defined for any finite complex reflection group, but no such definition exists for affine or double affine Hecke algebras. We propose to introduce the following algebra, which extends the definition of the degenerate DAHA of type $\mathfrak{g l}_{l}$ when $d=1$.

Definition 9.3. We denote by $\mathbf{H}_{t, \mathbf{c}}\left(\Gamma_{l}\right)$ the algebra $\mathbb{C}\left[x_{1}^{ \pm}, \ldots, x_{l}^{ \pm}\right] \otimes_{\mathbb{C}\left[x_{1}, \ldots, x_{l}\right]} \mathrm{H}_{t, \mathbf{c}}\left(\Gamma_{l}\right)$. (We can localize $\mathrm{H}_{t, \mathbf{c}}\left(\Gamma_{l}\right)$ at $\bigcup_{i=1}^{l}\left\{1, x_{i}, x_{i}^{2}, \ldots\right\}$ since this is an Ore set.)

We can obtain a presentation for $\mathbf{H}_{t, \mathbf{c}}\left(\Gamma_{l}\right)$ which is an affine version of Definition 9.1

Proposition 9.5. The algebra $\mathbf{H}_{t, \mathbf{c}}\left(\Gamma_{l}\right)$ can also be defined as the algebra generated by $x_{1}^{ \pm}, \ldots, x_{l}^{ \pm}, \gamma \in \Gamma_{l}$ and the pairwise commuting elements $u_{1}, \ldots, u_{l}$ subject to the following relations:

(1) The subalgebra generated by $x_{1}^{ \pm}, \ldots, x_{l}^{ \pm}, \gamma \in \Gamma_{l}$ is an epimorphic image of $\mathbb{C}\left[x_{1}^{ \pm}, \ldots, x_{l}^{ \pm}\right] \rtimes \Gamma_{l}$.

(2) The subalgebra generated by $u_{1}, \ldots, u_{l}$ and $\gamma \in \Gamma_{l}$ is an epimorphic image of $\mathrm{H}_{\kappa}\left(\Gamma_{l}\right)$.

(3) $\sigma_{0} u_{j}-\sigma_{0}\left(u_{j}\right) \sigma_{0}=-\kappa\left\langle\alpha_{0}, u_{j}\right\rangle \sum_{k=0}^{d-1} \zeta^{-k} \xi_{1}^{k} \xi_{l}^{-k}$, where $\sigma_{0}=x_{1} x_{l}^{-1} \sigma_{1 l}, u \in$ $\operatorname{span}_{\mathbb{C}}\left\{u_{1}, \ldots, u_{l}\right\}$ and $\alpha_{0}=u_{l}^{*}-u_{1}^{*},\left\{u_{1}^{*}, \ldots, u_{l}^{*}\right\}$ being the basis dual to $\left\{u_{1}, \ldots, u_{l}\right\} .\left(\sigma_{0}\left(u_{j}\right)\right.$ is defined in analogy with the action of the affine Weyl group on $\widehat{\mathfrak{h}}^{*}$.)

(4) $\pi u_{i}=u_{i+1} \pi$ if $i \neq l, \pi u_{l}=\left(u_{1}-t\right) \pi$, where $\pi=x_{1} \tau$ and $\tau=\sigma_{12} \sigma_{23} \cdots$ $\sigma_{l-1, l}$.

Corollary 9.2. For any $\mathbf{c}=\left(\kappa, c_{1}, \ldots, c_{d-1}\right), \mathbf{H}_{t, \mathbf{c}}\left(\Gamma_{l}\right) \cong \mathbf{H}_{t, \mathbf{c}=\left(\kappa, c_{a}=0\right)}\left(\Gamma_{l}\right)$.

An isomorphism $\mathbf{H}_{t, \mathbf{c}}\left(\Gamma_{l}\right) \stackrel{\sim}{\longrightarrow} \mathbf{H}_{t, \mathbf{c}=\left(\kappa, c_{a}=0\right)}\left(\Gamma_{l}\right)$ is given by

$$
y_{i} \mapsto y_{i}-\left(\sum_{k=1}^{d-1} d c_{k} \mathbf{e}_{k-1, i}\right) x_{i}^{-1}
$$

Proof. The second set of relations was already obtained in the proof of Proposition 9.1. We have to establish the third and fourth relations for $\mathcal{U}_{i}$. The conclusion will then follow from the PBW property of $\mathbf{H}_{t, \mathbf{c}}\left(\Gamma_{l}\right)$. Suppose first that $i \neq 1, l$. 


$$
\begin{aligned}
\sigma_{0} \mathcal{U}_{i}= & \frac{t}{2} \sigma_{0}+x_{i} x_{1} x_{l}^{-1} y_{i} \sigma_{1 l}+\kappa \sum_{2 \leq j<i} \sum_{k=0}^{d-1} \xi_{i}^{-k} \sigma_{i j} \xi_{i}^{k} \sigma_{0}+\kappa \sum_{k=0}^{d-1} \sigma_{0} \xi_{i}^{-k} \sigma_{1 i} \xi_{i}^{k} \\
& +\sum_{k=1}^{d-1} d c_{k} \mathbf{e}_{k, i} \sigma_{0} \\
= & \frac{t}{2} \sigma_{0}+x_{i} x_{1}\left[x_{l}^{-1}, y_{i}\right] \sigma_{1 l}+x_{i} x_{1} y_{i} x_{l}^{-1} \sigma_{1 l}+\kappa \sum_{2 \leq j<i} \sum_{k=0}^{d-1} \xi_{i}^{-k} \sigma_{i j} \xi_{i}^{k} \sigma_{0} \\
& +\kappa \sum_{k=0}^{d-1} x_{1} x_{l}^{-1} \xi_{i}^{-k} \sigma_{i l} \xi_{i}^{k} \sigma_{1 l}+\sum_{k=1}^{d-1} d c_{k} \mathbf{e}_{k, i} \sigma_{0} \\
= & \frac{t}{2} \sigma_{0}+\kappa x_{i} x_{1}\left(-x_{l}^{-1} \sum_{k=0}^{d-1} \zeta^{k} \xi_{l}^{-k} \sigma_{i l} \xi_{l}^{k} x_{l}^{-1}\right) \sigma_{1 l}+x_{i}\left[x_{1}, y_{i}\right] x_{l}^{-1} \sigma_{1 l}+x_{i} y_{i} x_{1} x_{l}^{-1} \sigma_{1 l} \\
& +\kappa \sum_{2 \leq j<i} \sum_{k=0}^{d-1} \xi_{i}^{-k} \sigma_{i j} \xi_{i}^{k} \sigma_{0}+\kappa \sum_{k=0}^{d-1} \zeta^{k} \xi_{i}^{-k} \sigma_{i l} \xi_{i}^{k} x_{1} x_{i}^{-1} \sigma_{1 l}+\sum_{k=1}^{d-1} d c_{k} \mathbf{e}_{k, i} \sigma_{0} \\
=- & \kappa \sum_{k=0}^{d-1} x_{i} \xi_{l}^{-k} x_{l}^{-1} \sigma_{i l} \xi_{l}^{k} x_{1} x_{l}^{-1} \sigma_{1 l}+\kappa x_{i}\left(\sum_{k=0}^{d-1} \zeta^{k} \xi_{1}^{-k} \sigma_{1 i} \xi_{1}^{k}\right) x_{l}^{-1} \sigma_{1 l}+\mathcal{U}_{i} \sigma_{0} \\
& \quad-\kappa \sum_{k=0}^{d-1} \xi_{i}^{-k} \sigma_{1 i} \xi_{i}^{k} \sigma_{0}+\kappa \sum_{k=0}^{d-1} \zeta^{k} \xi_{i}^{-k} \sigma_{i l} \xi_{i}^{k} x_{1} x_{i}^{-1} \sigma_{1 l} \\
= & \mathcal{U}_{i} \sigma_{0}
\end{aligned}
$$

The other case left to check is $i=1$. (The case $i=l$ follows from this one.)

$$
\begin{aligned}
\sigma_{0} \mathcal{U}_{1}= & \frac{t}{2} \sigma_{0}+x_{1} x_{l}^{-1} x_{l} y_{l} \sigma_{1 l}+x_{l}^{-1} \sum_{a=1}^{d-1} c_{a} \sum_{k=0}^{d-1} \zeta^{-k a} \xi_{l}^{k} x_{1} \sigma_{1 l} \\
= & \frac{t}{2} \sigma_{0}+x_{1} y_{l} \sigma_{1 l}+\sum_{a=1}^{d-1} c_{a} \sum_{k=0}^{d-1} \zeta^{-k(a-1)} \xi_{l}^{k} x_{l}^{-1} x_{1} \sigma_{1 l} \\
= & \frac{t}{2} \sigma_{0}+\left[x_{1}, y_{l}\right] \sigma_{1 l}+y_{l} x_{1} \sigma_{1 l}+\sum_{k=1}^{d-1} d c_{k} \mathbf{e}_{k-1, l} \sigma_{0} \\
= & \frac{t}{2} \sigma_{0}+\kappa \sum_{k=0}^{d-1} \zeta^{k} \xi_{1}^{-k} \sigma_{1 l} \xi_{1}^{k} \sigma_{1 l}+\left[y_{l}, x_{l}\right] \sigma_{0}+x_{l} y_{l} \sigma_{0}+\sum_{k=1}^{d-1} d c_{k} \mathbf{e}_{k-1, l} \sigma_{0} \\
= & \frac{t}{2} \sigma_{0}+\kappa \sum_{k=0}^{d-1} \zeta^{k} \xi_{1}^{-k} \xi_{l}^{k}+\left(t+\kappa \sum_{1 \leq j<l} \sum_{k=0}^{d-1} \xi_{l}^{-k} \sigma_{j l} \xi_{l}^{k}+\sum_{k=1}^{d-1} d c_{k}\left(\mathbf{e}_{k, l}-\mathbf{e}_{k-1, l}\right)\right) \sigma_{0} \\
& +x_{l} y_{l} \sigma_{0}+\sum_{k=1}^{d-1} d c_{k} \mathbf{e}_{k-1, l} \sigma_{0} \\
= & \kappa \sum_{k=0}^{d-1} \zeta^{k} \xi_{1}^{-k} \xi_{l}^{k}+\left(\mathcal{U}_{l}+t\right) \sigma_{0} .
\end{aligned}
$$


We now have to check the third relation involving $\pi$. First, let us assume that $i \neq l$. Then

$$
\begin{aligned}
\pi \mathcal{U}_{i}= & \frac{t}{2} \pi+x_{1}\left(x_{i+1} y_{i+1}+\kappa \sum_{2 \leq j<i+1} \sum_{k=0}^{d-1} \xi_{i+1}^{-k} \sigma_{i+1, j} \xi_{i+1}^{k}+\sum_{k=1}^{d-1} d c_{k} \mathbf{e}_{k, i+1}\right) \tau \\
= & \frac{t}{2} \pi+\left(x_{i+1}\left[x_{1}, y_{i+1}\right]+x_{i+1} y_{i+1} x_{1}\right. \\
& \left.\quad+\kappa \sum_{2 \leq j<i+1} \sum_{k=0}^{d-1} \xi_{i+1}^{-k} \sigma_{i+1, j} \xi_{i+1}^{k} x_{1}+\sum_{k=1}^{d-1} d c_{k} \mathbf{e}_{k, i+1} x_{1}\right) \tau \\
= & \frac{t}{2} \pi+\left(x_{i+1}\left(\kappa \sum_{k=0}^{d-1} \zeta^{k} \xi_{1}^{-k} \sigma_{1, i+1} \xi_{1}^{k}\right)+x_{i+1} y_{i+1} x_{1}\right. \\
+ & \left.\sum_{1 \leq j<i+1} \sum_{k=0}^{d-1} \xi_{i+1}^{-k} \sigma_{i+1, j} \xi_{i+1}^{k} x_{1}-\kappa \sum_{k=0}^{d-1} \xi_{i+1}^{-k} \sigma_{1, i+1} \xi_{i+1}^{k} x_{1}\right) \tau+\sum_{k=1}^{d-1} d c_{k} \mathbf{e}_{k, i+1} \pi \\
= & \mathcal{U}_{i+1} \pi . \quad
\end{aligned}
$$

Finally, we check the relation for $i=l$. Then

$$
\begin{aligned}
\pi \mathcal{U}_{l} & =\frac{t}{2} \pi+\left(x_{1}^{2} y_{1}+\kappa x_{1} \sum_{2 \leq j \leq l} \sum_{k=0}^{d-1} \xi_{1}^{-k} \sigma_{1 j} \xi_{1}^{k}+\sum_{a=1}^{d-1} c_{a} \sum_{k=0}^{d-1} \zeta^{-k a} x_{1} \xi_{1}^{k}\right) \tau \\
& =\frac{t}{2} \pi+\left(x_{1} y_{1} x_{1}+x_{1}\left[x_{1}, y_{1}\right]+\kappa x_{1} \sum_{2 \leq j \leq l} \sum_{k=0}^{d-1} \xi_{1}^{-k} \sigma_{1 j} \xi_{1}^{k}+\sum_{k=1}^{d-1} d c_{k} \mathbf{e}_{k+1,1} x_{1}\right) \tau \\
& =\frac{t}{2} \pi+x_{1} y_{1} \pi+\left(x_{1}\left(-t-\kappa \sum_{1<j \leq l} \sum_{k=0}^{d-1} \xi_{1}^{-k} \sigma_{1 j} \xi_{1}^{k}-\sum_{k=1}^{d-1} d c_{k}\left(\mathbf{e}_{k, 1}-\mathbf{e}_{k-1,1}\right)\right)\right. \\
& \left.+\kappa x_{1} \sum_{2 \leq j \leq l} \sum_{k=0}^{d-1} \xi_{1}^{-k} \sigma_{1 j} \xi_{1}^{k}\right) \tau+\sum_{k=1}^{d-1} d c_{k} \mathbf{e}_{k+1,1} \pi \\
& =\left(\mathcal{U}_{1}-t\right) \pi .
\end{aligned}
$$

9.2. Schur-Weyl duality in the trigonometric setting. In this section, we establish Schur-Weyl type equivalences for $\mathrm{H}_{\kappa}\left(\Gamma_{l}\right)$ and for $\mathbf{H}_{t, \mathbf{c}}\left(\Gamma_{l}\right)$. This will have applications for $\mathrm{H}_{t, \mathbf{c}}\left(\Gamma_{l}\right)$ in the next subsection. Recall that $\widehat{C}_{n-1}=\left(c_{i j}\right)_{0 \leq i, j \leq n-1}$ is the $n \times n$ Cartan matrix of affine type $\widehat{A}_{n-1}$.

Definition 9.4. Let $\lambda \in \mathbb{C}$. We denote by $Y_{\lambda}^{n, d}$ the algebra generated by the elements $X_{i, r, \mathbf{j}}^{ \pm}, H_{i, r, \mathbf{j}}^{ \pm}, 1 \leq i \leq n-1,0 \leq j \leq d-1, r \geq 0$ which satisfy the following relations: for any $1 \leq i_{1}, i_{2} \leq n-1, r_{1}, r_{2}, s \in \mathbb{Z}_{\geq 0}$ :

If $j_{1} \neq j_{2}$, then

$$
\begin{aligned}
& {\left[X_{i_{1}, r_{1}, \mathbf{j}_{1}}^{ \pm}, X_{i_{2}, r_{2}, \mathbf{j}_{2}}^{ \pm}\right]=\left[X_{i_{1}, r_{1}, \mathbf{j}_{1}}^{ \pm}, X_{i_{2}, r_{2}, \mathbf{j}_{2}}^{\mp}\right]=0} \\
& {\left[H_{i_{1}, r_{1}, \mathbf{j}_{1}}, X_{i_{2}, r_{2}, \mathbf{j}_{2}}^{ \pm}\right]=\left[H_{i_{1}, r_{1}, \mathbf{j}_{1}}, H_{i_{2}, r_{2}, \mathbf{j}_{2}}\right]=0,}
\end{aligned}
$$




$$
\begin{gathered}
{\left[H_{i_{1}, r_{1}, \mathbf{j}}, H_{i_{2}, r_{2}, \mathbf{j}}\right]=0, \quad\left[H_{i_{1}, 0, \mathbf{j}}, X_{i_{2}, s, \mathbf{j}}^{ \pm}\right]= \pm c_{i_{1}, i_{2}} X_{i_{2}, s, \mathbf{j}}^{ \pm},} \\
{\left[X_{i_{1}, r_{1}, \mathbf{j}}^{+}, X_{i_{2}, r_{2}, \mathbf{j}}^{-}\right]=\delta_{i_{1}, i_{2}} H_{i_{1}, r_{1}+r_{2}, \mathbf{j}},} \\
{\left[H_{i_{1}, r_{1}+1, \mathbf{j}}, X_{i_{2}, r_{2}, \mathbf{j}}^{ \pm}\right]-\left[H_{i_{1}, r_{1}, \mathbf{j}}, X_{i_{2}, r_{2}+1, \mathbf{j}}^{ \pm}\right]= \pm \frac{\lambda}{2} c_{i_{1}, i_{2}} S\left(H_{i_{1}, r_{1}, \mathbf{j}}, X_{i_{2}, r_{2}, \mathbf{j}}^{ \pm}\right),} \\
{\left[X_{i_{1}, r_{1}+1, \mathbf{j}}^{ \pm}, X_{i_{2}, r_{2}, \mathbf{j}}^{ \pm}\right]-\left[X_{i_{1}, r_{1}, \mathbf{j}}^{ \pm}, X_{i_{2}, r_{2}+1, \mathbf{j}}^{ \pm}\right]= \pm \frac{\lambda}{2} c_{i_{1}, i_{2}} S\left(X_{i_{1}, r_{1}, \mathbf{j}}^{ \pm}, X_{i_{2}, r_{2}, \mathbf{j}}^{ \pm}\right) .}
\end{gathered}
$$

For any $0 \leq j_{1}, j_{2} \leq d-1$,

$$
\sum_{\pi \in S_{m}}\left[X_{i_{1}, r_{\pi(1)}, \mathbf{j}_{1}}^{ \pm},\left[X_{i_{1}, r_{\pi(2)}, \mathbf{j}_{1}}^{ \pm}, \ldots,\left[X_{i_{1}, r_{\pi(m)}, \mathbf{j}_{1}}^{ \pm}, X_{i_{2}, s, \mathbf{j}_{2}}^{ \pm}\right] \ldots\right]\right]=0
$$

where $m=1-c_{i j}, r_{1}, \ldots, r_{m}, s \in \mathbb{Z}_{\geq 0}$.

Remark 9.2. The algebra $Y_{\lambda}^{n, d}$ is isomorphic to $\left(Y_{\lambda}^{n}\right)^{\otimes d}$, where $Y_{\lambda}^{n}=Y_{\lambda}^{n, d=1}$ is the Yangian (of finite type) for $\mathfrak{s l}_{n}$. The reason for using the notation above will become clear in the next definition. We will write $X_{i, \mathbf{j}}^{ \pm}, H_{i, \mathbf{j}}$ instead of $X_{i, 0, \mathbf{j}}^{ \pm}, H_{i, 0, \mathbf{j}}$.

Definition 9.5. We denote by $\widehat{\mathbf{Y}}_{\lambda, \underline{\beta}}^{n, d}, \underline{\beta}=\left(\beta_{0}, \beta_{1}, \ldots, \beta_{d-1}\right) \in \mathbb{C}^{d}$, the algebra generated by the elements $X_{i, r, \mathbf{j}}^{ \pm}, H_{i, r, \mathbf{j}}, 0 \leq i \leq n-1,0 \leq j \leq d-1, r \geq 0$ which satisfy the relations in Definition 9.4 (extended to $i_{1}=0$ or $i_{2}=0$ ) except that certain relations for $i_{1}=0$ or $i_{2}=0$ must be modified:

$$
\begin{aligned}
& {\left[H_{0, r_{1}+1, \mathbf{i}}, X_{0, r_{2}, \mathbf{j}}^{+}\right]-\left[H_{0, r_{1}, \mathbf{i}}, X_{0, r_{2}+1, \mathbf{j}}^{+}\right]=\delta_{i, j-1} \lambda S\left(H_{0, r_{1}, \mathbf{i}}, X_{0, r_{2}, \mathbf{j}}^{+}\right),} \\
& {\left[H_{0,0, \mathbf{i}}, X_{1, r, \mathbf{j}}^{ \pm}\right]=\mp \delta_{i, j-1} X_{1, r, \mathbf{j}}^{ \pm}, \quad\left[H_{0,0, \mathbf{i}}, X_{0, r, \mathbf{j}}^{+}\right]=2 \delta_{i, j-1} X_{0, r, \mathbf{j}}^{+},} \\
& {\left[X_{0, r, \mathbf{i}}^{+}, X_{0, s, \mathbf{j}}^{-}\right]=\delta_{i-1, j} H_{0, r+s, \mathbf{j}},} \\
& {\left[H_{1,0, \mathbf{i}}, X_{0, r, \mathbf{j}}^{-}\right]=\delta_{i, j+1} X_{0, r, \mathbf{j}}^{-}, \quad\left[H_{n-1,0, \mathbf{i}}, X_{0, r, \mathbf{j}}^{+}\right]=-\delta_{i+1, j} X_{0, r, \mathbf{j}}^{+} \text {, }} \\
& {\left[X_{0, r_{1}+1, \mathbf{j}}^{+}, X_{1, r_{2}, \mathbf{j}}^{+}\right]-\left[X_{0, r_{1}, \mathbf{j}}^{+}, X_{1, r_{2}+1, \mathbf{j}}^{+}\right]=\left(\beta_{j}-\lambda\right) X_{1, r_{2}, \mathbf{j}}^{+} X_{0, r_{1}, \mathbf{j}}^{+}-\beta_{j} X_{0, r_{1}, \mathbf{j}}^{+} X_{1, r_{2}, \mathbf{j}}^{+} \text {, }} \\
& {\left[X_{0, r_{1}+1, \mathbf{j}}^{-}, X_{1, r_{2}, \mathbf{j}+\mathbf{1}}^{-}\right]-\left[X_{0, r_{1}, \mathbf{j}}^{-}, X_{1, r_{2}+1, \mathbf{j}+\mathbf{1}}^{-}\right]=\beta_{j+1} X_{1, r_{2}, \mathbf{j}+\mathbf{1}}^{-} X_{0, r_{1}, \mathbf{j}}^{-}} \\
& -\left(\beta_{j+1}-\lambda\right) X_{0, r_{1}, \mathbf{j}}^{-} X_{1, r_{2}, \mathbf{j}+\mathbf{1}}^{-}, \\
& {\left[X_{0, r_{1}+1, \mathbf{j}+\mathbf{1}}^{+}, X_{n-1, r_{2}, \mathbf{j}}^{+}\right]-\left[X_{0, r_{1}, \mathbf{j}+\mathbf{1}}^{+}, X_{n-1, r_{2}+1, \mathbf{j}}^{+}\right]=\left(\beta_{j}-\lambda\right) X_{0, r_{1}, \mathbf{j}+\mathbf{1}}^{+} X_{n-1, r_{2}, \mathbf{j}}^{+}} \\
& -\beta_{j} X_{n-1, r_{2}, \mathbf{j}}^{+} X_{0, r_{1}, \mathbf{j}+\mathbf{1}}^{+} \text {, } \\
& {\left[X_{0, r_{1}+1, \mathbf{j}}^{-}, X_{n-1, r_{2}, \mathbf{j}}^{-}\right]-\left[X_{0, r_{1}, \mathbf{j}}^{-}, X_{n-1, r_{2}+1, \mathbf{j}}^{-}\right]=\beta_{j} X_{0, r_{1}, \mathbf{j}}^{-} X_{n-1, r_{2}, \mathbf{j}}^{-}} \\
& -\left(\beta_{j}-\lambda\right) X_{n-1, r_{2}, \mathbf{j}}^{-} X_{0, r_{1}, \mathrm{j}}^{-}, \\
& {\left[X_{n-1, r_{1}, \mathbf{j}_{1}}^{+}, X_{0, r_{2}, \mathbf{j}_{2}}^{+}\right]=0=\left[X_{0, r_{1}, \mathbf{j}_{1}}^{-}, X_{1, r_{2}, \mathbf{j}_{2}}^{-}\right] \text {if } j_{2} \neq j_{1}+1 \text {. }}
\end{aligned}
$$

From relations (73) and (75)-(78), we can obtain relations similar to (68).

Remark 9.3. Proposition 4.3 states that $\widehat{\mathbf{Y}}_{\lambda=0, \beta=0}^{n, d}$ is isomorphic to $\mathfrak{U}_{\mathfrak{s l}}\left(\mathbb{C}\left[u^{ \pm 1}, w\right]\right.$ $\rtimes \Gamma$ ) with $\Gamma$ acting on $u$ by $\xi(u)=\zeta u$ and trivially on $w$. The subalgebra of $\widehat{\mathbf{Y}}_{\lambda, \underline{\beta}}^{n, d}$ generated by the elements $X_{i, \mathbf{j}}^{ \pm}=X_{i, 0, \mathbf{j}}^{ \pm}$and $H_{i, \mathbf{j}}=H_{i, 0, \mathbf{j}}$ is isomorphic to ${\widehat{U} \widehat{\mathfrak{s l}}_{n}}_{n}\left(\mathbb{C}\left[u^{ \pm 1}\right] \rtimes \Gamma\right)$. It is explained in GHL that $\mathfrak{s l}_{n}\left(\mathbb{C}\left[u^{ \pm 1}\right] \rtimes \Gamma\right) \cong \mathfrak{s l}_{n d}\left(\mathbb{C}\left[t^{ \pm 1}\right]\right)$ with $t=u^{d}$ and thus $\mathfrak{s l}_{n}\left(\mathbb{C}\left[u^{ \pm 1}, w\right] \rtimes \Gamma\right) \cong \mathfrak{s l}_{n d}\left(\mathbb{C}\left[t^{ \pm 1}, w\right]\right)$.

Definition 9.6. A module $M$ over $\widehat{\mathbf{Y}}_{\lambda, \underline{\beta}}^{n, d}$ is said to have trivial central charge if the action of the element $\sum_{j=0}^{d-1} \sum_{i=0}^{n-1} H_{i, 0, \mathbf{j}}$ is trivial. 
Definition 9.7. The quotient of $\widehat{\mathbf{Y}}_{\lambda, \beta}^{n, d}$ by the ideal generated by the central element $\sum_{j=0}^{d-1} \sum_{i=0}^{n-1} H_{i, 0, \mathbf{j}}$ will be denoted $\mathbf{L} \overline{\mathbf{Y}}_{\lambda, \underline{\beta}}^{d}$.

The main results of this section are the next two propositions, which extend the results in Gu1.

Proposition 9.6. If $\lambda=\kappa d$, there exists a functor SW : $\mathrm{H}_{\kappa}\left(\Gamma_{l}\right)-\bmod _{R} \longrightarrow$ $Y_{\lambda}^{n, d}-\bmod _{L}^{l, \text { int }}$ given by $M \mapsto M \otimes_{\mathbb{C}\left[S_{l}\right]}\left(\mathbb{C}^{n}\right)^{\otimes l}$ which is an equivalence of categories if $l+1<n$.

Theorem 9.1. If $\lambda=\kappa d$ and $\beta_{j}=\frac{t}{2}-\frac{\lambda(n-2)}{4}+\frac{\left(c_{j}-c_{j+1}\right) d}{2}$ (where $c_{0}=0=c_{d}$ ), there exists a functor $\mathbb{S W}: \mathbf{H}_{t, \mathbf{c}}\left(\Gamma_{l}\right)-\bmod _{R} \longrightarrow \widehat{\mathbf{Y}}_{\lambda, \underline{\beta}}^{n, d}-\bmod _{L, \text { triv }}^{l, \text { int }}$ given by $M \mapsto$ $M \otimes_{\mathbb{C}\left[S_{l}\right]}\left(\mathbb{C}^{n}\right)^{\otimes l}$ which is an equivalence of categories if $l+2<n$.

Proof of Proposition 9.6. We use the presentation of $\mathrm{H}_{\kappa}\left(\Gamma_{l}\right)$ given in Proposition 9.4. Set $\omega_{i, \mathbf{j}}^{ \pm}= \pm \frac{1}{4} \sum_{\alpha \in \Delta^{+}} S\left(\left[X_{i, \mathbf{j}}^{ \pm}, X_{\alpha, \mathbf{j}}^{ \pm}\right], X_{\alpha, \mathbf{j}}^{\mp}\right)-\frac{1}{4} S\left(X_{i, \mathbf{j}}^{ \pm}, H_{i, \mathbf{j}}\right)$, where $X_{\alpha}^{ \pm}$ is the matrix $E_{i j} \in \mathfrak{s l}_{n}$ if $\pm \alpha=\epsilon_{i}-\epsilon_{j}$, and $J\left(E_{i}^{ \pm}\right)_{\mathbf{j}}=X_{i, 1, \mathbf{j}}^{ \pm}+\lambda \omega_{i, \mathbf{j}}^{ \pm}$. Setting $J\left(E_{i}^{ \pm}\right)_{\mathbf{j}}(m \otimes \mathbf{v})=\sum_{k=1}^{l} m \mathrm{y}_{k, j} \otimes E_{i}^{ \pm,(k)}(\mathbf{v})$ and $X_{i, \mathbf{j}}^{ \pm}(m \otimes \mathbf{v})=\sum_{k=1}^{l} m \mathbf{e}_{j, k} \otimes$ $E_{i}^{ \pm,(k)}(\mathbf{v})$ for $m \in M, \mathbf{v} \in\left(\mathbb{C}^{n}\right)^{\otimes l}$ gives $M \otimes_{\mathbb{C}\left[S_{l}\right]}\left(\mathbb{C}^{n}\right)^{\otimes l}$ a structure of a left module over $Y_{\lambda}^{n}$. This follows from Dr1] and equality (66). Since $\mathrm{y}_{k_{1}, j_{1}} \mathrm{y}_{k_{2}, j_{2}}=\mathrm{y}_{k_{2}, j_{2}} \mathrm{y}_{k_{1}, j_{1}}$ and $\mathbf{e}_{j_{1}, k_{1}} \mathbf{e}_{j_{2}, k_{2}}=0$ if $j_{1} \neq j_{2}$, we can conclude that these module structures commute, so we get a functor SW $: \mathrm{H}_{\kappa}\left(\Gamma_{l}\right)-\bmod _{R} \longrightarrow Y_{\lambda}^{n, d}-\bmod _{L}^{l, i n t}$.

Assume now that $l+1<n$ and let $N$ be an integrable $Y_{\lambda}^{n, d}$-module of level $l$. Since it is a direct sum of finite-dimensional modules over $\mathfrak{s l}_{n}(\mathbb{C}[\mathbb{Z} / d \mathbb{Z}])$, according to Proposition 6.2 (see also [ATY]), $N \cong M \otimes_{\mathbb{C}\left[S_{l}\right]}\left(\mathbb{C}^{n}\right)^{\otimes l}$ for a certain $\Gamma_{l}$-module $M$. It follows also from Dr1 (or by mimicking the argument in section 4.5 in ChPr1] ) that $J\left(E_{i}^{ \pm}\right)_{\mathbf{j}}=\sum_{k=1}^{l} m \mathrm{y}_{k, j} \otimes E_{i}^{ \pm,(j)}(\mathbf{v})$ and that $\mathrm{y}_{i, k}, 1 \leq i \leq l$ satisfy (66). That $\mathrm{y}_{i, k_{1}}$ and $\mathrm{y}_{j, k_{2}}$ commute as operators on $M$ if $k_{1} \neq k_{2}$ is a consequence of $\left[J\left(E_{i}^{ \pm}\right)_{\mathbf{k}_{1}}, J\left(E_{j}^{ \pm}\right)_{\mathbf{k}_{\mathbf{2}}}\right]=0$ if $k_{1} \neq k_{2}$.

Proof of Theorem 9.1. This is similar to the proof of theorem 5.4 in Gu1. Let $M \in \mathbf{H}_{t, \mathbf{c}}-\bmod _{R}$. We define a linear automorphism $T$ of $M \otimes_{\mathbb{C}\left[S_{l}\right]}\left(\mathbb{C}^{n}\right)^{\otimes l}$ by $T(m \otimes \mathbf{v})=m x_{1}^{-\delta_{i_{1} n}} \cdots x_{l}^{-\delta_{i_{l} n}} \otimes \mathbf{v}_{+1}$, where $\mathbf{v}_{+1}=v_{i_{1}+1} \otimes \cdots \otimes v_{i_{l}+1}$ if $\mathbf{v}=$ $v_{i_{1}} \otimes \cdots \otimes v_{i_{l}}$. $\widehat{\mathbf{Y}}_{\lambda, \underline{\beta}}^{n, d}$ admits an automorphism $\rho$ defined by

(80) $\rho\left(X_{i, r, \mathbf{j}}^{ \pm}\right)=\sum_{p=0}^{r}\left(\begin{array}{c}r \\ p\end{array}\right)\left(\frac{\lambda}{2}\right)^{p} X_{i-1, r-p, \mathbf{j}}^{ \pm}$if $i \neq 0,1$ and similarly for $\rho\left(H_{i, r, \mathbf{j}}\right)$,

$$
\begin{aligned}
\rho\left(X_{1, r, \mathbf{j}}^{+}\right) & =\sum_{p=0}^{r}\left(\begin{array}{c}
r \\
p
\end{array}\right) \beta_{j}^{p} X_{0, r-p, \mathbf{j}}^{+}, \rho\left(X_{0, r, \mathbf{j}}^{+}\right)=\sum_{p=0}^{r}\left(\begin{array}{c}
r \\
p
\end{array}\right) \beta_{j-1}^{p} X_{n-1, r-p, \mathbf{j}-\mathbf{1}}^{+}, \\
\rho\left(X_{1, r, \mathbf{j}}^{-}\right) & =\sum_{p=0}^{r}\left(\begin{array}{c}
r \\
p
\end{array}\right) \beta_{j}^{p} X_{0, r-p, \mathbf{j}-\mathbf{1}}^{-}, \rho\left(X_{0, r, \mathbf{j}}^{-}\right)=\sum_{p=0}^{r}\left(\begin{array}{c}
r \\
p
\end{array}\right) \beta_{j}^{p} X_{n-1, r-p, \mathbf{j}}^{-}, \\
\rho\left(H_{1, r, \mathbf{j}}\right) & =\sum_{p=0}^{r}\left(\begin{array}{c}
r \\
p
\end{array}\right) \beta_{j}^{p} H_{0, r-p, \mathbf{j}-\mathbf{1}}, \rho\left(H_{0, r, \mathbf{j}}\right)=\sum_{p=0}^{r}\left(\begin{array}{c}
r \\
p
\end{array}\right) \beta_{j}^{p} H_{n-1, r-p, \mathbf{j}} .
\end{aligned}
$$


Let $\varphi: Y_{\lambda}^{n, d} \longrightarrow \operatorname{End}_{\mathbb{C}}\left(M \otimes_{\mathbb{C}\left[S_{l}\right]}\left(\mathbb{C}^{n}\right)^{\otimes l}\right)$ be the algebra homomorphism coming from the $Y_{\lambda}^{d}$ module structure on $M \otimes_{\mathbb{C}\left[S_{l}\right]}\left(\mathbb{C}^{n}\right)^{\otimes l}$. The next lemma is the crucial part of the proof of Proposition 9.1.

Lemma 9.1. Under the same hypotheses on the parameters as in Theorem 9.1, the following equalities hold:

$$
\begin{aligned}
& \varphi\left(X_{i, r, \mathbf{j}}^{ \pm}\right)=T \circ \varphi\left(\rho\left(X_{i, r, \mathbf{j}}^{ \pm}\right)\right) \circ T^{-1} \text { if } 2 \leq i \leq n-1, \\
& \varphi\left(X_{1, r, \mathbf{j}}^{ \pm}\right)=T^{2} \circ \varphi\left(\rho^{2}\left(X_{1, r, \mathbf{j}}^{ \pm}\right)\right) \circ T^{-2}
\end{aligned}
$$

and similarly for $H_{i, r, \mathbf{j}}$ instead of $X_{i, r, \mathbf{j}}^{ \pm}$.

Proof. We give a direct, detailed proof of this equality similar to the first approach to lemma 6.2 in Gu1. We only need to prove it for $r=1$ and for $X_{i, 1, \mathbf{f}}^{+}$.

We have the following expressions for $\omega_{1, \mathrm{f}}^{+}$and $\omega_{n-1, \mathrm{f}}^{+}$:

$$
\begin{gathered}
\omega_{1, \mathbf{f}}^{+}=\frac{1}{4} \sum_{j=3}^{n} S\left(E_{j 2, \mathbf{f}}, E_{1 j, \mathbf{f}}\right)-\frac{1}{4} S\left(E_{12, \mathbf{f}}, H_{1, \mathbf{f}}\right), \\
\omega_{n-1, \mathbf{f}}^{+}=-\frac{1}{4} \sum_{j=1}^{n-2} S\left(E_{j n, \mathbf{f}}, E_{n-1, j, \mathbf{f}}\right)-\frac{1}{4} S\left(E_{n-1, n, \mathbf{f}}, H_{n-1, \mathbf{f}}\right) .
\end{gathered}
$$

Fix $\mathbf{v}=v_{i_{1}} \otimes \cdots \otimes v_{i_{l}} \in\left(\mathbb{C}^{n}\right)^{\otimes l}$. Suppose that $j_{1}, \ldots, j_{p}\left(\right.$ resp. $\left.\eta_{1}, \ldots, \eta_{e}\right)$ are exactly the values of $j$ (resp. of $\eta$ ) such that $i_{j}=n$ (resp. $\left.i_{\eta}=n-1\right)$. Then $T^{2}(m \otimes \mathbf{v})=m x_{j_{1}}^{-1} \cdots x_{j_{p}}^{-1} x_{\eta_{1}}^{-1} \cdots x_{\eta_{e}}^{-1} \otimes \mathbf{v}_{+2}$. Set $x_{j_{1}, \ldots, j_{p}}^{-1}=x_{j_{1}}^{-1} \cdots x_{j_{p}}^{-1}, x_{\eta_{1}, \ldots, \eta_{e}}^{-1}=$ $x_{\eta_{1}}^{-1} \cdots x_{\eta_{e}}^{-1}$. Since $X_{1,1, \mathbf{f}}^{+}=J\left(X_{1}^{+}\right)_{\mathbf{f}}-\lambda \omega_{1, \mathbf{f}}^{+}$, we obtain:

$$
\begin{aligned}
X_{1,1, \mathbf{f}}^{+}\left(T^{2}(m \otimes \mathbf{v})\right)= & \sum_{k=1}^{l} m x_{j_{1}, \ldots, j_{p}}^{-1} x_{\eta_{1}, \ldots, \eta_{e}}^{-1} \mathcal{Y}_{k} \mathbf{e}_{f, k} \otimes E_{12}^{(k)}\left(\mathbf{v}_{+2}\right) \\
& -\frac{\lambda}{4} \sum_{j=3}^{n} S\left(E_{j 2, \mathbf{f}}, E_{1 j, \mathbf{f}}\right)\left(m x_{j_{1}, \ldots, j_{p}}^{-1} x_{\eta_{1}, \ldots, \eta_{e}}^{-1} \otimes \mathbf{v}_{+2}\right) \\
& +\frac{\lambda}{4} S\left(E_{12, \mathbf{f}}, H_{1, \mathbf{f}}\right)\left(m x_{j_{1}, \ldots, j_{p}}^{-1} x_{\eta_{1}, \ldots, \eta_{e}}^{-1} \otimes \mathbf{v}_{+2}\right) .
\end{aligned}
$$

In the summation (85), we can assume that $k=j_{r}$ for some $r$, since otherwise $E_{12}^{(k)}\left(\mathbf{v}_{+2}\right)=0$.

$$
\begin{aligned}
\text { [85) }= & \sum_{r=1}^{p} \sum_{b=1}^{e} m x_{j_{1}, \ldots, j_{p}}^{-1} x_{\eta_{1}}^{-1} \cdots x_{\eta_{b-1}}^{-1}\left[x_{\eta_{b}}^{-1}, \mathcal{Y}_{j_{r}}\right] x_{\eta_{b+1}}^{-1} \cdots x_{\eta_{e}}^{-1} \mathbf{e}_{f, j_{r}} \otimes E_{12}^{\left(j_{r}\right)}\left(\mathbf{v}_{+2}\right) \\
& +\sum_{r=1}^{p} \sum_{b=1}^{p} m x_{j_{1}}^{-1} \cdots x_{j_{b-1}}^{-1}\left[x_{j_{b}}^{-1}, \mathcal{Y}_{j_{r}}\right] x_{j_{b+1}}^{-1} \cdots x_{j_{p}}^{-1} x_{\eta_{1}, \ldots, \eta_{e}}^{-1} \mathbf{e}_{f, j_{r}} \otimes E_{12}^{\left(j_{r}\right)}\left(\mathbf{v}_{+2}\right) \\
& +\sum_{k=1}^{l} m \mathcal{Y}_{k} x_{j_{1}, \ldots, j_{p}}^{-1} x_{\eta_{1}, \ldots, \eta_{e}}^{-1} \mathbf{e}_{f, k} \otimes E_{12}^{(k)}\left(\mathbf{v}_{+2}\right) .
\end{aligned}
$$


The last expression is equal to $T^{2}\left(J\left(X_{n-1}^{+}\right)_{\mathbf{f}-\mathbf{1}}(m \otimes \mathbf{v})\right)$. As for the term on line (87) above, it is equal to $\frac{\lambda}{4} T^{2}\left(S\left(E_{n-1, n, \mathbf{f}-\mathbf{1}}, H_{n-1, \mathbf{f}-\mathbf{1}}\right)(m \otimes \mathbf{v})\right)$. Therefore,

$$
X_{1,1, \mathbf{f}}^{+}\left(T^{2}(m \otimes \mathbf{v})\right)=(\underline{88})+(\underline{89})+(\underline{86})+T^{2}\left(X_{n-1,1, \mathbf{f}-\mathbf{1}}^{+}(m \otimes \mathbf{v})\right)-(\underline{102}) .
$$

(The expression (102) appears explicitly below.)

We need to decompose the sums (88) and (89). Sum (88) equals

$$
\begin{gathered}
=-\frac{\kappa}{2} \sum_{r=1}^{p} \sum_{b=1}^{e} \sum_{k=0}^{b-1} m x_{j_{1}, \ldots, j_{p}}^{-1} x_{\eta_{1}}^{-1} \cdots x_{\eta_{b-1}}^{-1}\left(x_{\eta_{b}}^{-1}+\zeta^{-k} x_{j_{r}}^{-1}\right) \xi_{\eta_{b}}^{-k} \sigma_{\eta_{b}, j_{r}} \xi_{\eta_{b}}^{k} x_{\eta_{b+1}}^{-1} \cdots \\
x_{\eta_{e}}^{-1} \mathbf{e}_{f, j_{r}} \otimes E_{12}^{\left(j_{r}\right)}\left(\mathbf{v}_{+2}\right) \\
(91)=-\frac{\kappa}{2} \sum_{r=1}^{p} \sum_{b=1}^{e} m x_{j_{1}, \ldots, j_{p}}^{-1} x_{\eta_{1}}^{-1} \cdots x_{\eta_{b-1}}^{-1} x_{\eta_{b}}^{-1} x_{\eta_{b+1}}^{-1} \cdots x_{\eta_{e}}^{-1} \mathbf{e}_{f, \eta_{b}} \mathbf{e}_{f, j_{r}} \otimes E_{12}^{\left(\eta_{b}\right)}\left(\sigma_{\eta_{b}, j_{r}}\left(\mathbf{v}_{+2}\right)\right) \\
\text { (92) } \quad-\frac{\kappa}{2} \sum_{r=1}^{p} \sum_{b=1}^{e} m x_{j_{1}, \ldots, j_{p}}^{-1} x_{\eta_{1}}^{-1} \cdots x_{\eta_{b-1}}^{-1} x_{j_{r}}^{-1} x_{\eta_{b+1}}^{-1} \cdots x_{\eta_{e}}^{-1} \mathbf{e}_{f, \eta_{b}} \mathbf{e}_{f+1, j_{r}} \otimes E_{12}^{\left(\eta_{b}\right)}\left(\sigma_{\eta_{b}, j_{r}}\left(\mathbf{v}_{+2}\right)\right) .
\end{gathered}
$$

As for the sum (89), it equals

$$
\begin{aligned}
& =\sum_{a=1}^{p} \sum_{\substack{b=1 \\
b \neq a}}^{p} m x_{j_{1}}^{-1} \cdots x_{j_{b-1}}^{-1}\left[x_{j_{b}}^{-1}, \mathcal{Y}_{j_{a}}\right] x_{j_{b+1}}^{-1} \cdots x_{j_{p}}^{-1} x_{\eta_{1}, \ldots, \eta_{e}}^{-1} \mathbf{e}_{f, j_{a}} \otimes E_{12}^{\left(j_{a}\right)}\left(\mathbf{v}_{+2}\right) \\
& +\sum_{a=1}^{p} m x_{j_{1}}^{-1} \cdots x_{j_{a-1}}^{-1}\left[x_{j_{a}}^{-1}, \mathcal{Y}_{j_{a}}\right] x_{j_{a+1}}^{-1} \cdots x_{j_{p}}^{-1} x_{\eta_{1}, \ldots, \eta_{e}}^{-1} \mathbf{e}_{f, j_{a}} \otimes E_{12}^{\left(j_{a}\right)}\left(\mathbf{v}_{+2}\right) \\
& =-\frac{\kappa}{2} \sum_{a=1}^{p} \sum_{\substack{b=1 \\
b \neq a}}^{p} \sum_{k=0}^{d-1} m x_{j_{1}, \ldots, j_{b-1}}^{-1}\left(x_{j_{b}}^{-1}+\zeta^{-k} x_{j_{a}}^{-1}\right) \xi_{j_{b}}^{-k} \sigma_{j_{b}, j_{a}} \xi_{j_{b}}^{k} x_{j_{b+1}, \ldots, j_{p}}^{-1} x_{\eta_{1}, \ldots, \eta_{e}}^{-1} \mathbf{e}_{f, j_{a}} \\
& \otimes E_{12}^{j a}\left(\mathbf{v}_{+2}\right) \\
& +\sum_{a=1}^{p} m x_{j_{1}, \ldots, j_{a-1}}^{-1}\left(\frac{\kappa}{2} \sum_{\substack{q=1 \\
q \neq j_{a}}}^{l} \sum_{k=0}^{d-1}\left(x_{j_{a}}^{-1}+\zeta^{-k} x_{q}^{-1}\right) \xi_{j_{a}}^{-k} \sigma_{j_{a}, q} \xi_{j_{a}}^{k}+t x_{j_{a}}^{-1}\right. \\
& \text { (93) } \left.+\frac{x_{j_{a}}^{-1}}{2} \sum_{b=1}^{d-1} d c_{b}\left(\mathbf{e}_{b+1, j_{a}}-\mathbf{e}_{b-1, j_{a}}\right)\right) x_{j_{a+1}, \ldots, j_{p}}^{-1} x_{\eta_{1}, \ldots, \eta_{e}}^{-1} \mathbf{e}_{f, j_{a}} \otimes E_{12}^{\left(j_{a}\right)}\left(\mathbf{v}_{+2}\right) \\
& \text { (94) }=-\frac{\kappa d}{2} \sum_{a=1}^{p} \sum_{\substack{b=1 \\
b \neq a}}^{p}\left(m x_{j_{1}, \ldots, j_{b-1}}^{-1} x_{j_{b}}^{-1} \sigma_{j_{a}, j_{b}}\left(x_{j_{b+1}, \ldots, j_{p}}^{-1}\right) x_{\eta_{1}, \ldots, \eta_{e}}^{-1} \mathbf{e}_{f, j_{b}} \mathbf{e}_{f-\delta(a>b), j_{a}}\right. \\
& \otimes E_{12}^{\left(j_{b}\right)}\left(\sigma_{j_{a}, j_{b}}\left(\mathbf{v}_{+2}\right)\right) \\
& \text { (95) } \left.+m x_{j_{1}, \ldots, j_{b-1}}^{-1} x_{j_{a}}^{-1} \sigma_{j_{b}, j_{a}}\left(x_{j_{b+1}, \ldots, j_{p}}^{-1}\right) x_{\eta_{1}, \ldots, \eta_{e}}^{-1} \mathbf{e}_{f, j_{b}} \mathbf{e}_{f+1-\delta(a>b), j_{a}} \otimes E_{12}^{\left(j_{b}\right)}\left(\sigma_{j_{a}, j_{b}}\left(\mathbf{v}_{+2}\right)\right)\right) \\
& \text { (96) }+\sum_{a=1}^{p}\left(t+\frac{1}{2} \sum_{b=1}^{d-1} d c_{b}\left(\mathbf{e}_{b, j_{a}}-\mathbf{e}_{b-2, j_{a}}\right)\right) m x_{j_{1}, \ldots, j_{p}}^{-1} x_{\eta_{1}, \ldots, \eta_{e}}^{-1} \mathbf{e}_{f, j_{a}} \otimes E_{12}^{\left(j_{a}\right)}\left(\mathbf{v}_{+2}\right) \\
& \text { (97) }+\frac{\kappa d}{2} \sum_{a=1}^{p} \sum_{\substack{q=1 \\
q \neq j_{a}}}^{l} m x_{j_{1}, \ldots, j_{a-1}}^{-1} x_{j_{a}}^{-1} \mathbf{e}_{f, j_{a}} \sigma_{j_{a}, q}\left(x_{j_{a+1}, \ldots, j_{p}}^{-1} x_{\eta_{1}, \ldots, \eta_{e}}^{-1}\right) \mathbf{e}_{f, q} \otimes E_{12}^{(q)}\left(\sigma_{j_{a}, q}\left(\mathbf{v}_{+2}\right)\right) \\
& \text { (98) }+\frac{\kappa d}{2} \sum_{a=1}^{p} \sum_{\substack{q=1 \\
q \neq j_{a}}}^{l} m x_{j_{1}, \ldots, j_{a-1}}^{-1} x_{q}^{-1} \mathbf{e}_{f-1, j_{a}} \sigma_{j_{a}, q}\left(x_{j_{a+1}, \ldots, j_{p}}^{-1} x_{\eta_{1}, \ldots, \eta_{e}}^{-1}\right) \mathbf{e}_{f, q} \otimes E_{12}^{(q)}\left(\sigma_{j_{a}, q}\left(\mathbf{v}_{+2}\right)\right) \text {, }
\end{aligned}
$$

where $\delta(a>b)=1$ if $a>b$ and $=0$ if $a \leq b$.

We now focus on $\omega_{1, \mathbf{f}}^{+}\left(T^{2}(m \otimes \mathbf{v})\right)$ and $T^{2}\left(\omega_{n-1, \mathbf{f}-\mathbf{1}}^{+}(m \otimes \mathbf{v})\right)$. Earlier, we have used the equality $\lambda \omega_{1, \mathbf{f}}^{+}\left(T^{2}(m \otimes \mathbf{v})\right)=-$ (86) - (87). We can decompose (86) by 
considering the cases when $E_{1 j}$ and $E_{j 2}$ act on the same tensorand and on different ones:

$$
\begin{aligned}
-(\underline{86})= & \lambda\left(\frac{n-2}{4}\right) \sum_{k=1}^{l} m x_{j_{1}, \ldots, j_{p}}^{-1} x_{\eta_{1}, \ldots, \eta_{e}}^{-1} \mathbf{e}_{f, k} \otimes E_{12}^{(k)}\left(\mathbf{v}_{+2}\right) \\
& +\frac{\lambda}{2} \sum_{j=3}^{n} \sum_{\substack{q=1 \\
i_{q}+2=j}}^{l} \sum_{b=1}^{p} m x_{j_{1}, \ldots, j_{p}}^{-1} x_{\eta_{1}, \ldots, \eta_{e}}^{-1} \mathbf{e}_{f, q} \mathbf{e}_{f, j_{b}} \otimes E_{12}^{(q)}\left(\sigma_{q, j_{b}}\left(\mathbf{v}_{+2}\right)\right), \\
\lambda & T^{2}\left(\omega_{n-1, \mathbf{f}-\mathbf{1}}^{+}(m \otimes \mathbf{v})\right)=-\frac{\lambda}{4} T^{2}\left(\sum_{j=1}^{n-2} S\left(E_{j n, \mathbf{f}-\mathbf{1}}, E_{n-1, j, \mathbf{f}-\mathbf{1}}\right)(m \otimes \mathbf{v})\right) \\
& -\frac{\lambda}{4} T^{2}\left(S\left(E_{n-1, n, \mathbf{f}-\mathbf{1}}, H_{n-1, \mathbf{f}-\mathbf{1}}\right)(m \otimes \mathbf{v})\right) .
\end{aligned}
$$

We observe that $-(87)=(102)$. As with (86), we can decompose (101):

$$
\begin{aligned}
\text { (101) }= & -\lambda\left(\frac{n-2}{4}\right) \sum_{k=1}^{l} m x_{j_{1}, \ldots, j_{p}}^{-1} x_{\eta_{1}, \ldots, \eta_{e}}^{-1} \mathbf{e}_{f, k} \otimes\left(E_{n-1, n}^{(k)}(\mathbf{v})\right)_{+2} \\
& -\frac{\lambda}{2} T^{2}\left(\sum_{j=1}^{n-2} \sum_{\substack{q=1 \\
i_{q}=j}}^{l} \sum_{b=1}^{p} m \mathbf{e}_{f-1, q} \mathbf{e}_{f-1, j_{b}} \otimes E_{n-1, n}^{(q)}\left(\sigma_{q, j_{b}}(\mathbf{v})\right)\right) .
\end{aligned}
$$

We note that (99) $-(103)=\lambda\left(\frac{n-2}{2}\right) T^{2}\left(E_{n-1, n, \mathbf{f}-\mathbf{1}}(m \otimes \mathbf{v})\right)$ and

$$
\text { (104) }=-\frac{\lambda}{2} \sum_{j=1}^{n-2} \sum_{\substack{q=1 \\ i_{q}=j}}^{l} \sum_{b=1}^{p} m \mathbf{e}_{f-1, q} \mathbf{e}_{f-1, j_{b}} x_{j_{1}, \ldots, j_{p}}^{-1} x_{j_{b}} x_{q}^{-1} x_{\eta_{1}, \ldots, \eta_{e}}^{-1} \otimes E_{12}^{(q)}\left(\sigma_{q, j_{b}}\left(\mathbf{v}_{+2}\right)\right) \text {. }
$$

To obtain the last expression, note that $i_{q} \neq j_{a}, \eta_{h}$ for any $a, h$ since $i_{q}=j$ and we consider values of $j$ different from $n$ and $n-1$.

We now decompose the sums (97) and (98) into three different sums. In the first case, $q=j_{b} \neq j_{a}$; in the second case, $q=\eta_{h}$; in the third case $q \neq j_{a}, \eta_{h}$ for any $a, h$. The sum (97) equals

$$
\begin{aligned}
& (105) \frac{\kappa d}{2} \sum_{a=1}^{p} \sum_{\substack{b=1 \\
b \neq a}}^{p} m x_{j_{1}, \ldots, j_{a}}^{-1} \mathbf{e}_{f, j_{a}} \sigma_{j_{a}, j_{b}}\left(x_{j_{a+1}, \ldots, j_{p}}^{-1}\right) x_{\eta_{1}, \ldots, \eta_{e}}^{-1} \mathbf{e}_{f, j_{b}} \otimes E_{12}^{\left(j_{b}\right)}\left(\sigma_{j_{a}, j_{b}}\left(\mathbf{v}_{+2}\right)\right) \\
& (106)+\frac{\kappa d}{2} \sum_{a=1}^{p} \sum_{h=1}^{e} m x_{j_{1}, \ldots, j_{a}}^{-1} \mathbf{e}_{f, j_{a}} x_{j_{a+1}, \ldots, j_{p}}^{-1} x_{\eta_{1}, \ldots, \eta_{e}}^{-1} x_{\eta_{h}} x_{j_{a}}^{-1} \mathbf{e}_{f, \eta_{h}} \otimes E_{12}^{\left(\eta_{h}\right)}\left(\sigma_{j_{a}, \eta_{h}}\left(\mathbf{v}_{+2}\right)\right) \\
& (107) \quad+\frac{\kappa d}{2} \sum_{a=1}^{p} \sum_{\substack{q=1 \\
q \neq j_{d}, \eta_{h}}}^{l} m x_{j_{1}, \ldots, j_{a}}^{-1} \mathbf{e}_{f, j_{a}} x_{j_{a+1}, \ldots, j_{p}}^{-1} x_{\eta_{1}, \ldots, \eta_{e}}^{-1} \mathbf{e}_{f, q} \otimes E_{12}^{(q)}\left(\sigma_{j_{a}, q}\left(\mathbf{v}_{+2}\right)\right)
\end{aligned}
$$

As for the sum (98), it equals

$$
\begin{aligned}
& \frac{\kappa d}{2} \sum_{\substack{a=1 \\
b \neq 1 \\
b \neq a}}^{p} m x_{j_{1}, \ldots, j_{a-1}}^{-1} x_{j_{b}}^{-1} \mathbf{e}_{f-1, j_{a}} \sigma_{j_{a}, j_{b}}\left(x_{j_{a+1}, \ldots, j_{p}}^{-1}\right) x_{\eta_{1}, \ldots, \eta_{e}}^{-1} \mathbf{e}_{f, j_{b}} \\
& \otimes E_{12}^{\left(j_{b}\right)}\left(\sigma_{j_{a}, j_{b}}\left(\mathbf{v}_{+2}\right)\right)
\end{aligned}
$$




$$
\begin{aligned}
& +\frac{\kappa d}{2} \sum_{a=1}^{p} \sum_{h=1}^{e} m x_{j_{1}, \ldots, j_{a-1}}^{-1} x_{\eta_{h}}^{-1} \mathbf{e}_{f-1, j_{a}} x_{j_{a+1}, \ldots, j_{p}}^{-1} x_{\eta_{1}, \ldots, \eta_{e}}^{-1} x_{\eta_{h}} x_{j_{a}}^{-1} \mathbf{e}_{f, \eta_{h}} \\
& \otimes E_{12}^{\left(\eta_{h}\right)}\left(\sigma_{j_{a}, \eta_{h}}\left(\mathbf{v}_{+2}\right)\right) \\
& +\frac{\kappa d}{2} \sum_{a=1}^{p} \sum_{\substack{q=1 \\
q \neq j_{d}, \eta_{h}}}^{l} m x_{j_{1}, \ldots, j_{a-1}}^{-1} x_{q}^{-1} \mathbf{e}_{f-1, j_{a}} x_{j_{a+1}, \ldots, j_{p}}^{-1} x_{\eta_{1}, \ldots, \eta_{e}}^{-1} \mathbf{e}_{f-1, q} \\
& \otimes E_{12}^{(q)}\left(\sigma_{j_{a}, q}\left(\mathbf{v}_{+2}\right)\right) .
\end{aligned}
$$

The following equalities hold since we are assuming that $\lambda=\kappa d$ :

$$
(87)=-(102),(107)=(100),(110)=-(104),(109)=-(91),
$$

(92) $=-(106),(94)=-(108) \cdot(95)=-(105)$.

Using our assumption that $\beta_{f}=\frac{t}{2}-\frac{\lambda(n-2)}{4}+\frac{\left(c_{f}-c_{f+1}\right) d}{2}$, we can prove that $X_{1,1, \mathbf{f}}^{+}\left(T^{2}(m \otimes \mathbf{v})\right)=T^{2}\left(\left(X_{n-1,1, \mathbf{f}-\mathbf{1}}^{+}+\left(\beta_{f}+\beta_{f-1}\right) X_{n-1, \mathbf{f}-\mathbf{1}}^{+}\right)(m \otimes \mathbf{v})\right):$

$X_{1,1, \mathbf{f}}^{+}\left(T^{2}(m \otimes \mathbf{v})\right)-T^{2}\left(X_{n-1,1, \mathbf{f}-\mathbf{1}}^{+}(m \otimes \mathbf{v})\right)$

$=(85)+(86)+(87)-T^{2}\left(X_{n-1,1, \mathbf{f}-1}^{+}(m \otimes \mathbf{v})\right)$

$=(88)+(89)+T^{2}\left(J\left(X_{n-1}^{+}\right)_{\mathbf{f}-\mathbf{1}}(m \otimes \mathbf{v})\right)-T^{2}\left(X_{n-1,1, \mathbf{f}-\mathbf{1}}^{+}(m \otimes \mathbf{v})\right)+(86)+(87)$

$=(91)+\cdots+(\underline{98})+(186)+(87)+$ (101) + (102)

$=(\underline{10})+\cdots+(\underline{96})+(105)+\cdots+(110)-(99)-(100)$

$+(\underline{87})+(\underline{102})+(\underline{103})+(104)$

$=(\underline{(87)}+(\underline{102}))+(\underline{(91)}+(\underline{109}))+(\underline{(92)}+(\underline{106}))+(\underline{(94)}+(\underline{108}))+(\underline{95})+(\underline{105})$

$+(\underline{96})-(\underline{99})+(-\underline{100})+(\underline{107}))+(\underline{104})+(\underline{110}))+(\underline{103})$

$=(\underline{96})-(99)+(103)$

$=\left(t-\lambda\left(\frac{n-2}{2}\right)+\frac{d\left(c_{f-1}-c_{f+1}\right)}{2}\right) T^{2}\left(E_{n-1, n, \mathbf{f}-\mathbf{1}}(m \otimes \mathbf{v})\right)$

$=\left(\beta_{f}+\beta_{f-1}\right) T^{2}\left(X_{n-1, \mathbf{f}-\mathbf{1}}^{+}(m \otimes \mathbf{v})\right)$.

Using the equalities (84), we can extend $\varphi$ to an algebra homomorphism $\widehat{\mathbf{Y}}_{\lambda, \underline{\beta}}^{n, d} \longrightarrow$ $\operatorname{End}_{\mathbb{C}}\left(M \otimes_{\mathbb{C}\left[S_{l}\right]}\left(\mathbb{C}^{n}\right)^{\otimes l}\right)$ (also denoted $\left.\varphi\right)$ by setting $\varphi\left(X_{0, r, \mathbf{j}}^{ \pm}\right)=T \circ \varphi\left(\rho\left(X_{0, r, \mathbf{j}}^{ \pm}\right) \circ T^{-1}\right.$. We have thus constructed a functor $\mathbf{H}_{t, \mathbf{c}}\left(\Gamma_{l}\right)-\bmod _{R} \longrightarrow \widehat{\mathbf{Y}}_{\lambda, \underline{\beta}}^{n, d}-\bmod _{L, t r i v}^{\text {int,l }}$. The proof that it is an equivalence of categories when $l+2<n$ follows the same approach as the second part of the proof of theorem 5.4 in Gu1].

Let $N \in \widehat{\mathbf{Y}}_{\lambda, \underline{\beta}}^{n, d}-\bmod _{L, t r i v}^{\text {int, }}$. Since $N$ is a module over $Y_{\lambda}^{d}$ and over $\mathfrak{U} \widehat{\mathfrak{s l}}_{n}\left(\mathbb{C}\left[u^{ \pm 1}\right] \rtimes\right.$ $\Gamma)$, it is equal to $M \otimes_{\mathbb{C}\left[S_{l}\right]}\left(\mathbb{C}^{n}\right)^{\otimes l}$ for some $\Gamma_{l}$-module $M$, which is also a module over $\mathrm{H}_{\kappa}\left(\Gamma_{l}\right)$ and $\mathbb{C}\left[x_{1}^{ \pm 1}, \ldots, x_{l}^{ \pm}\right] \rtimes \Gamma_{l}$. We have to show that these two structures can be glued together to give $M$ a structure of a module over $\mathbf{H}_{t, \mathbf{c}}\left(\Gamma_{l}\right)$. 
Fix $1 \leq j, k \leq l, j \neq k$. We choose $\mathbf{v}$ to be the following generator of $\left(\mathbb{C}^{n}\right)^{\otimes l}$ as a $\mathfrak{U}_{\mathfrak{s l}}{ }_{n}$-module: $\mathbf{v}=v_{i_{1}} \otimes v_{i_{2}} \otimes \cdots \otimes v_{i_{l}}$ where $i_{b}=b+3$ if $b<j, b \neq k, i_{b}=b+2$ if $b>j, b \neq k, i_{j}=2$ and $i_{k}=1$.

We can express $\omega_{2, \mathbf{f}}^{-}$explicitly in the following way:

$$
\left.\omega_{2}^{-}\right|_{\left(\mathbb{C}^{n}\right) \otimes l}=-\frac{1}{4} \sum_{\substack{b=1 \\ b \neq 2,3}}^{n} \operatorname{sign}(2-b) S\left(E_{3 b, \mathbf{f}}, E_{b 2, \mathbf{f}}\right)-\frac{1}{4} S\left(E_{32, \mathbf{f}}, H_{2, \mathbf{f}}\right) .
$$

We find that $\left(X_{2,1, \mathbf{f}_{\mathbf{1}}}^{-} X_{0, \mathbf{f}_{\mathbf{2}}}^{+}-X_{0, \mathbf{f}_{\mathbf{2}}}^{+} X_{2,1, \mathbf{f}_{1}}^{-}\right)(m \otimes \mathbf{v})$ equals

$$
\begin{aligned}
& \sum_{r=1}^{l} \sum_{s=1}^{l}\left(m x_{r} \mathbf{e}_{f_{2}-1, r} \mathbf{e}_{f_{1}, s} \mathcal{Y}_{s} \otimes E_{32}^{(s)} E_{n 1}^{(r)}(\mathbf{v})-m \mathbf{e}_{f_{1}, s} \mathcal{Y}_{s} x_{r} \mathbf{e}_{f_{2}-1, r} \otimes E_{n 1}^{(r)} E_{32}^{(s)}(\mathbf{v})\right) \\
& -\lambda\left[\omega_{2, \mathbf{f}_{1}}^{-}, X_{0, \mathbf{f}_{2}}^{+}\right](m \otimes \mathbf{v}) \\
= & m\left[x_{k} \mathbf{e}_{f_{2}-1, k}, \mathcal{Y}_{j} \mathbf{e}_{f_{1}, j}\right] \otimes E_{32}^{(j)} E_{n 1}^{(k)}(\mathbf{v})-\delta_{f_{1}, f_{2}} \frac{\lambda}{2} m x_{j} \mathbf{e}_{f_{2}-1, j} \mathbf{e}_{f_{1}, k} \otimes E_{31}^{(k)} E_{n 2}^{(j)}(\mathbf{v}) \\
& -\delta_{f_{1}, f_{2}-1} \frac{\lambda}{2} m x_{k} \mathbf{e}_{f_{2}-1, k} \mathbf{e}_{f_{1}, j} \otimes E_{31}^{(k)} E_{n 2}^{(j)}(\mathbf{v}) \\
= & m\left(\left[x_{k} \mathbf{e}_{f_{2}-1, k}, \mathcal{Y}_{j} \mathbf{e}_{f_{1}, j}\right]-\delta_{f_{1}, f_{2}} \frac{\lambda}{2} x_{j} \sigma_{j k} \mathbf{e}_{f_{2}-1, k} \mathbf{e}_{f_{1}, j}-\delta_{f_{1}, f_{2}-1} \frac{\lambda}{2} x_{k} \sigma_{j k} \mathbf{e}_{f_{1}, j} \mathbf{e}_{f_{2}-1, k}\right) \\
& \otimes \widetilde{\mathbf{v}},
\end{aligned}
$$

where $\widetilde{\mathbf{v}}=E_{32}^{(j)} E_{n 1}^{(k)}(\mathbf{v})$. We know from relation (69) that $\left[X_{2,1, \mathbf{f}_{\mathbf{1}}}^{-}, X_{0, \mathbf{f}_{2}}^{+}\right]=0$, so the last expression is equal to 0 . Since $\widetilde{\mathbf{v}}$ is a generator of $\left(\mathbb{C}^{n}\right)^{\otimes l}$ as a $\mathfrak{U l s l}_{n}$-module, it follows, from Lemma 6.1 and our assumption that $\lambda=\kappa d$, that

$$
\begin{aligned}
& m\left(\left[x_{k} \mathbf{e}_{f_{2}-1, k}, \mathcal{Y}_{j} \mathbf{e}_{f_{1}, j}\right]\right. \\
& \left.\quad-\delta_{f_{1}, f_{2}} \frac{\kappa d}{2} x_{j} \sigma_{j k} \mathbf{e}_{f_{2}-1, k} \mathbf{e}_{f_{1}, j}-\delta_{f_{1}, f_{2}-1} \frac{\kappa d}{2} x_{k} \sigma_{j k} \mathbf{e}_{f_{1}, j} \mathbf{e}_{f_{2}-1, k}\right)=0 .
\end{aligned}
$$

Summing over $f_{1}, f_{2}=0,1, \ldots, d-1$ and using $\sum_{f=0}^{d-1} \mathbf{e}_{f, k} \mathbf{e}_{f, j}=\frac{1}{d} \sum_{f=0}^{d-1} \xi_{k}^{-f} \xi_{j}^{f}$, $\sum_{f=0}^{d-1} \mathbf{e}_{f-1, k} \mathbf{e}_{f, j}=\frac{1}{d} \sum_{f=0}^{d-1} \zeta^{-f} \xi_{k}^{-f} \xi_{j}^{f}$ yields relation (60).

We now consider the relation between $x_{k}$ and $\mathcal{Y}_{k}$. Setting

$$
\nu_{1, \mathbf{f}}=\frac{1}{4} \sum_{i=3}^{n} S\left(E_{1 i, \mathbf{f}}, E_{i 1, \mathbf{f}}\right)+\frac{1}{2} S\left(E_{12, \mathbf{f}}, E_{21, \mathbf{f}}\right)-\frac{1}{4} \sum_{i=3}^{n} S\left(E_{2 i, \mathbf{f}}, E_{i 2, \mathbf{f}}\right)-\frac{1}{2} H_{1, \mathbf{f}}^{2},
$$

we have that $H_{i, 1, \mathbf{j}}(m \otimes \mathbf{v})=J\left(H_{i}\right)_{\mathbf{j}}(m \otimes \mathbf{v})-\lambda \nu_{1, \mathbf{f}}(m \otimes \mathbf{v})$ with $J\left(H_{i}\right)(m \otimes \mathbf{v})=$ $\sum_{k=1}^{l} m \mathcal{Y}_{k} \mathbf{e}_{f, k} \otimes H_{i}^{(k)}(\mathbf{v})$. 
Fix $k, 1 \leq k \leq l$. We now choose $\mathbf{v}$ to be equal to $\mathbf{v}=v_{i_{1}} \otimes \cdots \otimes v_{i_{l}}$ with $i_{b}=b+2$ if $b<k, i_{b}=b+1$ if $b>k$ and $i_{k}=1$. Note that $v_{i_{b}} \neq 2, n, n-1 \forall b$ since $l+1<n-1$ by assumption. We obtain:

$$
\begin{gathered}
{\left[E_{n 1}^{(r)}, \nu_{1}\right](\mathbf{v})=\frac{1}{2} \sum_{b=3}^{n} E_{n b}^{(r)} E_{b 1}^{(k)}(\mathbf{v})=\frac{1}{2} \sigma_{k r} E_{n 1}^{(k)}(\mathbf{v}) \text { if } r \neq k} \\
{\left[E_{n 1}^{(k)}, \nu_{1}\right](\mathbf{v})=\left(\frac{n-2}{4}\right) E_{n 1}^{(k)}(\mathbf{v}) .}
\end{gathered}
$$

We need (111) to obtain equation (112) below. We compute that $\left(H_{1,1, \mathbf{f}} X_{0, \mathbf{f}}^{+}\right.$ $\left.X_{0, \mathbf{f}}^{+} H_{1,1, \mathbf{f}}\right)(m \otimes \mathbf{v})$ equals

$$
\begin{aligned}
\sum_{r=1}^{l} & \sum_{s=1}^{l} m \mathbf{e}_{f, r} x_{r} \mathbf{e}_{f, s} \mathcal{Y}_{s} \otimes H_{1}^{s} E_{n 1}^{(r)}(\mathbf{v})-\sum_{s=1}^{l} \sum_{r=1}^{l} m \mathbf{e}_{f, s} \mathcal{Y}_{s} \mathbf{e}_{f, r} x_{r} \otimes E_{n 1}^{(r)} H_{1}^{(s)}(\mathbf{v}) \\
& -\lambda\left[\nu_{1, \mathbf{f}}, X_{0, \mathbf{f}}^{+}\right](m \otimes \mathbf{v}) \\
= & -m \mathbf{e}_{f, k} \mathcal{Y}_{k} \mathbf{e}_{f, k} x_{k} \otimes E_{n 1}^{(k)} H_{1}^{(k)}(\mathbf{v})+\frac{\lambda}{2} \sum_{\substack{r=1 \\
r \neq k}}^{l} m x_{r} \mathbf{e}_{f-1, r} \mathbf{e}_{f, k} \otimes \sigma_{k r} E_{n 1}^{(k)}(\mathbf{v}) \\
& +\lambda\left(\frac{n-2}{4}\right) m \mathbf{e}_{f, k} x_{k} \otimes E_{n 1}^{(k)}(\mathbf{v}) \\
(112)= & -m \mathcal{Y}_{k} \mathbf{e}_{f, k} x_{k} \mathbf{e}_{f-1, k} \otimes \widetilde{\mathbf{v}}+\frac{\lambda}{2} \sum_{\substack{r=1 \\
r \neq k}}^{l} m x_{r} \sigma_{k r} \mathbf{e}_{f-1, k} \mathbf{e}_{f, r} \otimes \widetilde{\mathbf{v}} \\
& +\lambda\left(\frac{n-2}{4}\right) m x_{k} \mathbf{e}_{f-1, k} \otimes \widetilde{\mathbf{v}},
\end{aligned}
$$

where $\widetilde{\mathbf{v}}=E_{n 1}^{(k)}(\mathbf{v})$. We want to obtain a similar relation with $H_{1,1, \mathbf{f}}$ replaced by $H_{n-1,1, \mathbf{f}}$.

From the definition of $\nu_{n-1, \mathbf{f}-\mathbf{1}}$,

$$
\begin{aligned}
\nu_{n-1, \mathbf{f}-\mathbf{1}}= & \frac{1}{4} \sum_{b=1}^{n-2} S\left(E_{b n, \mathbf{f}-\mathbf{1}}, E_{n b, \mathbf{f}-\mathbf{1}}\right)+\frac{1}{2} S\left(E_{n-1, n, \mathbf{f}-\mathbf{1}}, E_{n, n-1, \mathbf{f}-\mathbf{1}}\right) \\
& -\frac{1}{4} \sum_{b=1}^{n-2} S\left(E_{b, n-1, \mathbf{f}-\mathbf{1}}, E_{n-1, b, \mathbf{f}-\mathbf{1}}\right)-\frac{1}{2} H_{n-1, \mathbf{f}-\mathbf{1}}^{2} .
\end{aligned}
$$

We can check that $\left[E_{n 1}^{(r)}, \nu_{n-1}\right](\mathbf{v})=0$ if $r \neq k$ and 


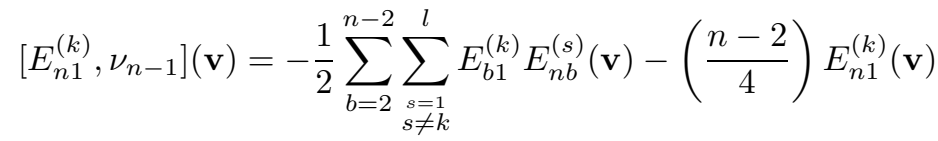

$$
\begin{aligned}
& =-\frac{1}{2} \sum_{\substack{s=1 \\
s \neq k}}^{l} \sigma_{k s} E_{n 1}^{(k)}(\mathbf{v})-\left(\frac{n-2}{4}\right) E_{n 1}^{(k)}(\mathbf{v}) .
\end{aligned}
$$

The equation (113) allows us to compute that $\left[H_{n-1,1, \mathbf{f}-\mathbf{1}}, X_{0, \mathbf{f}}^{+}\right](m \otimes \mathbf{v})$ equals:

$$
\begin{aligned}
= & \sum_{r, s=1}^{l}\left(m \mathbf{e}_{f, r} x_{r} \mathbf{e}_{f-1, s} \mathcal{Y}_{s} \otimes H_{n-1}^{(s)} E_{n 1}^{(r)}(\mathbf{v})-m \mathbf{e}_{f-1, s} \mathcal{Y}_{s} \mathbf{e}_{f, r} x_{r} \otimes E_{n 1}^{(r)} H_{n-1}^{(s)}(\mathbf{v})\right) \\
& -\lambda\left[\nu_{n-1, \mathbf{f}-\mathbf{1}}, X_{0, \mathbf{f}}^{+}\right](m \otimes \mathbf{v}) \\
= & m x_{k} \mathbf{e}_{f-1, k} \mathcal{Y}_{k} \mathbf{e}_{f-1, k} \otimes H_{n-1}^{(k)} E_{n 1}^{(k)}(\mathbf{v}) \\
(114) \quad & -\frac{\lambda}{2} \sum_{\substack{r=1 \\
r \neq k}}^{l} m x_{k}\left(\sigma_{k r} \mathbf{e}_{f-1, k} \mathbf{e}_{f-1, r}\right) \otimes E_{n 1}^{(k)}(\mathbf{v})-\left(\frac{n-2}{4}\right) \mathbf{e}_{f-1, k} \otimes E_{n 1}^{(k)}(\mathbf{v}) .
\end{aligned}
$$

From relations (67), (74), (75) and (77) in $\widehat{\mathbf{Y}}_{\lambda, \underline{\beta}}^{n, d}$, we know that

$$
\begin{aligned}
& X_{0,1, \mathbf{f}}^{+}=-\left[H_{1,1, \mathbf{f}}, X_{0, \mathbf{f}}^{+}\right]+\left(\beta_{f}-\lambda\right) H_{1, \mathbf{f}} X_{0, \mathbf{f}}^{+}-\beta_{f} X_{0, \mathbf{f}}^{+} H_{1, \mathbf{f}}
\end{aligned}
$$

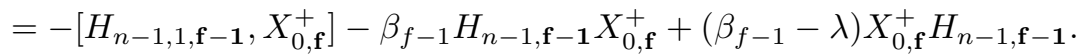

Applying these two expressions for $X_{0,1, \mathbf{f}}^{+}$to $m \otimes \mathbf{v}$, using equalities (112), (114) and the fact that $H_{1, \mathbf{f}} X_{0, \mathbf{f}}^{+}(\mathbf{v})=0$ and $X_{0, \mathbf{f}}^{+} H_{n-1, \mathbf{f}-\mathbf{1}}(\mathbf{v})=0$, we obtain that the expression

$$
\begin{array}{r}
m \mathcal{Y}_{k} x_{k} \mathbf{e}_{f-1, k} \otimes \widetilde{\mathbf{v}}-\frac{\lambda}{2} \sum_{\substack{r=1 \\
r \neq k}}^{l} m x_{r} \sigma_{k r} \mathbf{e}_{f-1, k} \mathbf{e}_{f, r} \widetilde{\mathbf{v}}-\lambda\left(\frac{n-2}{4}\right) m x_{k} \mathbf{e}_{f-1, k} \otimes \widetilde{\mathbf{v}} \\
-\beta_{f} X_{0, \mathbf{f}}^{+} H_{1, \mathbf{f}}(m \otimes \mathbf{v})
\end{array}
$$

equals

$$
\begin{aligned}
& m x_{k} \mathcal{Y}_{k} \mathbf{e}_{f-1, k} \otimes \widetilde{\mathbf{v}}+\frac{\lambda}{2} \sum_{\substack{r=1 \\
r \neq k}}^{l} m x_{k} \sigma_{k r} \mathbf{e}_{f-1, k} \mathbf{e}_{f-1, r} \otimes \widetilde{\mathbf{v}} \\
& \quad+\lambda\left(\frac{n-2}{4}\right) m x_{k} \mathbf{e}_{f-1, k} \otimes \widetilde{\mathbf{v}}-\beta_{f-1} H_{n-1, \mathbf{f}-\mathbf{1}} X_{0, \mathbf{f}}^{+}(m \otimes \mathbf{v}) .
\end{aligned}
$$

Therefore,

$$
\begin{aligned}
m\left[x_{k}, \mathcal{Y}_{k}\right] \mathbf{e}_{f-1, k} \otimes \widetilde{\mathbf{v}}+\frac{\lambda}{2} \sum_{\substack{r=1 \\
r \neq k}}^{l} m\left(x_{r} \mathbf{e}_{f, k}+x_{k} \mathbf{e}_{f-1, k}\right) \sigma_{k r} \mathbf{e}_{f-1, k} \otimes \widetilde{\mathbf{v}} \\
+\lambda\left(\frac{n-2}{2}\right) m x_{k} \mathbf{e}_{f-1, k} \otimes \widetilde{\mathbf{v}}+\left(\beta_{f-1}+\beta_{f}\right) m x_{k} \mathbf{e}_{f-1, k} \otimes \widetilde{\mathbf{v}}=0 .
\end{aligned}
$$


Since $\widetilde{\mathbf{v}}$ is a generator of $\left(\mathbb{C}^{n}\right)^{\otimes l}$ as a $\mathfrak{U}_{\mathfrak{s l}}$-module, it follows from Lemma 6.1 and our assumption that $\beta_{f}=\frac{t}{2}-\frac{\lambda(n-2)}{4}+\frac{\left(c_{f}-c_{f+1}\right) d}{2}, \lambda=\kappa d$ that

$$
\begin{array}{r}
m\left(\left[x_{k}, \mathcal{Y}_{k}\right] \mathbf{e}_{f-1, k}+\frac{\kappa d}{2} \sum_{\substack{r=1 \\
r \neq k}}^{l}\left(x_{r} \mathbf{e}_{f, k}+x_{k} \mathbf{e}_{f-1, k}\right) \sigma_{k r} \mathbf{e}_{f-1, k}\right. \\
\left.+\left(t+\frac{d}{2}\left(c_{f-1}-c_{f+1}\right)\right) x_{k} \mathbf{e}_{f-1, k}\right)
\end{array}
$$

vanishes. Taking the sum over $f=0, \ldots, d-1$, we obtain that

$m\left(\left[x_{k}, \mathcal{Y}_{k}\right]+t x_{k}+\frac{\kappa}{2} \sum_{\substack{j=1 \\ j \neq k}}^{l} \sum_{s=0}^{d-1}\left(x_{k}+\zeta^{s} x_{j}\right) \xi_{k}^{-s} \sigma_{j k} \xi_{k}^{s}+\frac{1}{2} \sum_{a=1}^{d-1} d c_{a}\left(\mathbf{e}_{a+1, k}-\mathbf{e}_{a-1, k}\right) x_{k}\right)=0$.

Therefore, we have proved that the $\mathrm{H}_{\kappa}\left(\Gamma_{l}\right)$ - and the $\mathbb{C}\left[x_{1}^{ \pm 1}, \ldots, x_{l}^{ \pm 1}\right] \rtimes \Gamma_{l}$-module structure on $M$ can be glued to yield a right module over $\mathbf{H}_{t, \mathbf{c}}\left(\Gamma_{l}\right)$. To prove that $\mathbb{S W}$ is an equivalence, we are left to show that it is fully faithful. That $\mathbb{S W}$ is injective on morphisms is true because this is true for the Schur-Weyl duality functor between $\mathbb{C}\left[S_{l}\right]$ and $\mathfrak{U}_{\mathfrak{s l}}$, so suppose that $f: \mathbb{S W}\left(M_{1}\right) \longrightarrow \mathbb{S W}\left(M_{2}\right)$ is a $\widehat{\mathbf{Y}}_{\lambda, \underline{\beta}}^{n, d}$-homomorphism. Schur-Weyl equivalence for $\mathfrak{s l}_{n}\left(\mathbb{C}\left[u^{ \pm 1}\right]\right)$ and $Y_{\lambda}^{n}$ says that it must be of the form $f\left(m_{1} \otimes \mathbf{v}\right)=g\left(m_{1}\right) \otimes \mathbf{v}, \forall m_{1} \in M_{1}$, where $g \in \operatorname{Hom}_{\mathbb{C}}\left(M_{1}, M_{2}\right)$ is a linear map which is also a homomorphism of right $\mathbb{C}\left[x_{1}^{ \pm 1}, \ldots, x_{l}^{ \pm 1}\right]$ - and $\mathrm{H}_{\kappa}\left(\Gamma_{l}\right)$ modules. Since $\mathbf{H}_{t, \mathbf{c}}\left(\Gamma_{l}\right)$ is generated by these two subalgebras, $g$ is even a homomorphism of $\mathbf{H}_{t, \mathbf{c}}\left(\Gamma_{l}\right)$-modules. Therefore, $f=\mathbb{S W}(g)$ and this completes the proof of Proposition 9.1.

It was explained after Proposition 9.5 that $\mathbf{H}_{t, \mathbf{c}}\left(\Gamma_{l}\right) \cong \mathbf{H}_{t, \mathbf{c}\left(\kappa, c_{a}=0\right)}\left(\Gamma_{l}\right)$. Therefore, it is reasonable to expect that $\widehat{\mathbf{Y}}_{\lambda, \beta}^{n, d}$ depends only on two parameters. This is confirmed in the next proposition. Let $\widetilde{\beta}=2 \sum_{i=0}^{d-1} \beta_{i}$ and let $\widehat{\mathbf{Y}}_{\lambda, \widetilde{\beta}}^{n, d}$ be the algebra defined as $\widehat{\mathbf{Y}}_{\lambda, \beta}^{n, d}$ with all the $\beta_{j}$ and $\lambda-\beta_{j}$ replaced by $\frac{\lambda}{2}$ in relations (75) (78) except that (75) and (76) for the cases $j=0$ and $j=d-1$, respectively, must be replaced by

$$
\begin{aligned}
& {\left[X_{0, r_{1}+1, \mathbf{0}}^{+}, X_{1, r_{2}, \mathbf{0}}^{+}\right]-\left[X_{0, r_{1}, \mathbf{0}}^{+}, X_{1, r_{2}+1, \mathbf{0}}^{+}\right]} \\
& \quad=\left(\widetilde{\beta}-\frac{\lambda}{2}\right) X_{1, r_{2}, \mathbf{0}}^{+} X_{0, r_{1}, \mathbf{0}}^{+}-\left(\widetilde{\beta}+\frac{\lambda}{2}\right) X_{0, r_{1}, \mathbf{0}}^{+} X_{1, r_{2}, \mathbf{0}}^{+}, \\
& {\left[X_{0, r_{1}+1, \mathbf{d}-\mathbf{1}}^{-}, X_{1, r_{2}, \mathbf{0}}^{-}\right]-\left[X_{0, r_{1}, \mathbf{d}-\mathbf{1}}^{-}, X_{1, r_{2}+1, \mathbf{0}}^{-}\right]} \\
& \quad=\left(\widetilde{\beta}+\frac{\lambda}{2}\right) X_{1, r_{2}, \mathbf{0}}^{-} X_{0, r_{1}, \mathbf{d}-\mathbf{1}}^{-}-\left(\widetilde{\beta}-\frac{\lambda}{2}\right) X_{0, r_{1}, \mathbf{d}-\mathbf{1}}^{-} X_{1, r_{2}, \mathbf{0}}^{-} .
\end{aligned}
$$

Proposition 9.7. The following formulas define an algebra isomorphism $\Psi: \widehat{\mathbf{Y}}_{\lambda, \underline{\beta}}^{n, d} \stackrel{\sim}{\longrightarrow}$ $\widehat{\mathbf{Y}}_{\lambda, \widetilde{\beta}}^{n, d}$ for any $\lambda, \underline{\beta}$ :

$$
\begin{gathered}
\Psi\left(X_{0, r, \mathbf{j}}^{-}\right)=\sum_{s=0}^{r}\left(\begin{array}{c}
r \\
s
\end{array}\right) 2^{s}\left(\beta_{0}+\cdots+\beta_{j}-j \frac{\lambda}{2}\right)^{s} X_{0, r-s, \mathbf{j}}^{-} \text {for } 0 \leq j \leq d-1, \\
\Psi\left(X_{0, r, \mathbf{j}}^{+}\right)=\sum_{s=0}^{r}\left(\begin{array}{c}
r \\
s
\end{array}\right) 2^{s}\left(\beta_{0}+\cdots+\beta_{j-1}-(j-1) \frac{\lambda}{2}\right)^{s} X_{0, r-s, \mathbf{j}}^{+} \text {for } 1 \leq j \leq d,
\end{gathered}
$$




$$
\Psi\left(H_{0, r, \mathbf{j}}\right)=\sum_{s=0}^{r}\left(\begin{array}{c}
r \\
s
\end{array}\right) 2^{s}\left(\beta_{0}+\cdots+\beta_{j}-j \frac{\lambda}{2}\right)^{s} H_{0, r-s, \mathbf{j}} \text { for } 0 \leq j \leq d-1 .
$$

For $1 \leq i \leq n-1$,

$$
\Psi\left(X_{i, r, \mathbf{j}}^{ \pm}\right)=\sum_{s=0}^{r}\left(\begin{array}{l}
r \\
s
\end{array}\right)\left(2 \beta_{0}+\cdots+2 \beta_{j-1}+\beta_{j}-(2 j-1) \frac{\lambda}{2}\right)^{s} X_{i, r-s, \mathbf{j}}^{ \pm} \text {for } 0 \leq j \leq d-1
$$

and similarly for $H_{i, r, \mathbf{j}}$

In view of Proposition 4.3 and the comments preceding it, the next corollary is not surprising.

Corollary 9.3. $\widehat{\mathbf{Y}}_{\lambda, \underline{\beta}}^{n, d}$ is isomorphic to an affine Yangian $\widehat{\mathbf{Y}}_{\lambda, \beta^{\prime}}^{n d}$ for $\mathfrak{s l}_{n d}$.

$\widehat{\mathbf{Y}}_{\lambda, \beta^{\prime}}^{n d}$ is generated by $X_{i, r}^{ \pm}, H_{i, r}$ for $0 \leq i \leq n d-1, r \in \mathbb{Z}_{\geq 0}$ and an isomorphism sends $X_{i, r, \mathbf{j}}^{+} \mapsto X_{j n+i, r}^{+}$for $0 \leq i \leq n-1,0 \leq j \leq d-1, X_{i, r, \mathbf{j}}^{-} \mapsto X_{j n+i, r}^{-}$for $1 \leq i \leq n-1,0 \leq j \leq d-1, X_{0, r, \mathbf{j}}^{-} \mapsto X_{(j+1) n, r}^{-}$for $0 \leq j \leq d-1$ (and $\left.X_{d n, r}^{-}=X_{0, r}^{-}\right)$.

In the definition of affine Yangians in $\mathrm{Gu} 2$, the parameter $\beta$ appears in relations involving $X_{0, r}^{ \pm}, X_{1, s}^{ \pm}$and also $X_{0, r}^{ \pm}, X_{n-1, s}^{ \pm}$. However, the relations before Proposition 9.7 involve only $X_{0, r}^{ \pm}$and $X_{1, s}^{ \pm}$. This is not a contradiction; affine Yangians can also be defined in such a way: this is explained in section 3 in [FFNR.

As is explained in Gu2, when $\lambda=0$ and $\beta \neq 0$, the affine Yangian $\widehat{\mathbf{Y}}_{\lambda=0, \beta}^{n}$ is isomorphic to $\widehat{\mathfrak{s l}}_{n}\left(\mathcal{D}\left(\mathbb{C}^{\times}\right)\right)$, the universal central extension of $\mathfrak{s l}_{n}$ over the ring $\mathcal{D}\left(\mathbb{C}^{\times}\right)$of differential operators on the torus $\mathbb{C}^{\times}$. Corollary 9.3 then implies that $\widehat{\mathbf{Y}}_{\lambda=0, \beta}^{n, d}$ is isomorphic to $\widehat{\mathfrak{s l}}_{n d}\left(\mathcal{D}\left(\mathbb{C}^{\times}\right)\right)$when $\beta^{1} \neq 0$. This is a consequence of the following observation: $\mathcal{D}\left(\mathbb{C}^{\times}\right) \rtimes \Gamma \cong M_{d}\left(\mathcal{D}\left(\mathbb{C}^{\times}\right)\right)$, an isomorphism being given on the generators of $\mathcal{D}\left(\mathbb{C}^{\times}\right) \rtimes \Gamma$ by

$$
w \mapsto\left(\begin{array}{ccccc}
d \partial & 0 & \cdots & \cdots & 0 \\
0 & d \partial-1 & 0 & \cdots & \vdots \\
\vdots & \ddots & \ddots & \ddots & \vdots \\
0 & \ddots & 0 & d \partial-(d-2) & 0 \\
0 & 0 & \cdots & 0 & d \partial-(d-1)
\end{array}\right), u \mapsto\left(\begin{array}{ccccc}
0 & 1 & 0 & \cdots & 0 \\
\vdots & 0 & 1 & \ddots & \vdots \\
\vdots & \ddots & \ddots & \ddots & 0 \\
0 & \ddots & \ddots & 0 & 1 \\
z & 0 & \cdots & 0 & 0
\end{array}\right)
$$

and the generator $\xi$ of $\mathbb{Z} / d \mathbb{Z}$ goes to the diagonal matrix with entries $1, \zeta^{-1}, \zeta^{-2}, \ldots$, $\zeta^{-(d-1)}$. Here, we view $\mathcal{D}\left(\mathbb{C}^{\times}\right) \rtimes \Gamma$ as the algebra generated by $w, u, u^{-1}$ and $\xi^{j} \in \Gamma, j=0, \ldots, d-1$, with the relations $w u-u w=u, \xi w=w \xi$ and $\xi u=\zeta u \xi$; we view $\mathcal{D}\left(\mathbb{C}^{\times}\right)$as the algebra generated by $\partial, z$ with the relation $\partial z-z \partial=z$. Note that $u^{d}$ is mapped to the diagonal matrix with entries $z, z, \ldots, z$ on the diagonal.

9.3. $\mathrm{D}_{\beta, \mathbf{b}}^{n}(\mathbb{Z} / d \mathbb{Z})$ as a subalgebra of $\mathbf{L} \mathbf{Y}_{\lambda, \underline{\beta}}^{d}$. Since $\mathbf{H}_{t, \mathbf{c}}\left(\Gamma_{l}\right) \hookrightarrow \mathbf{H}_{t, \mathbf{c}}\left(\Gamma_{l}\right)$, we may expect $\mathrm{D}_{\beta, \mathbf{b}}^{n}(\mathbb{Z} / d \mathbb{Z})$ to be isomorphic to a subalgebra of $\mathbf{L} \mathbf{Y}_{\lambda, \underline{\beta}}^{d}$. This is indeed true.

Definition 9.8. Let $\mathbb{L}_{\lambda, \underline{\beta}}^{d}$ be the subalgebra of $\mathbf{L} \mathbf{Y}_{\lambda, \underline{\beta}}^{d}$ generated by $X_{i, r, \mathbf{j}}^{ \pm}, H_{i, r, \mathbf{j}}$, $X_{0, r, \mathbf{j}}^{+}, X_{0, r+1, \mathbf{j}}^{-}$for $1 \leq i \leq n-1, r \geq 0,0 \leq j \leq d-1$. 
In order to prove Theorem 9.3 below, we need to construct a Schur-Weyl functor between $\mathbb{L}_{\lambda, \underline{\beta}}^{d}$ and $\mathrm{H}_{t, \mathbf{c}}\left(\Gamma_{l}\right)$. As in Gu1], we have to compute how $X_{0,1, \mathbf{j}}^{-}$acts on $M \otimes_{\mathbb{C}\left[S_{l}\right]}\left(\mathbb{C}^{n}\right)^{\otimes l}$ for a right $\mathrm{H}_{t, \mathbf{c}}\left(\Gamma_{l}\right)$-module $M$. For $m \in M$, we find that $\left[J\left(H_{1}\right)_{\mathbf{f}}, X_{0, \mathbf{f}-\mathbf{1}}^{-}\right](m \otimes \mathbf{v})$ equals

$$
\begin{aligned}
& \sum_{k=1}^{l} m x_{k}^{-1} \mathcal{Y}_{k} \mathbf{e}_{f, k} \otimes E_{1 n}^{(k)}(\mathbf{v})+\sum_{\substack{j, k=1 \\
j \neq k}}^{l} m\left[x_{k}^{-1}, \mathcal{Y}_{j}\right] \mathbf{e}_{f-1, k} \mathbf{e}_{f, j} \otimes H_{1}^{(j)} E_{1 n}^{(k)}(\mathbf{v}) \\
& =\frac{1}{2} \sum_{k=1}^{l} m\left(y_{k}+x_{k}^{-1} y_{k} x_{k}\right) \mathbf{e}_{f, k} \otimes E_{1 n}^{(k)}(\mathbf{v}) \\
& -\frac{\kappa}{2} \sum_{\substack{j, k=1 \\
j \neq k}}^{l} m\left(\sum_{i=0}^{d-1}\left(\zeta^{-i} x_{j}^{-1}+x_{k}^{-1}\right) \xi_{k}^{-i} \sigma_{j k} \xi_{k}^{i}\right) \mathbf{e}_{f, k} \mathbf{e}_{f, j} \otimes H_{1}^{(j)} E_{1 n}^{(k)}(\mathbf{v}) \\
& =\sum_{k=1}^{l} m y_{k} \mathbf{e}_{f, k} \otimes E_{1 n}^{(k)}(\mathbf{v})+\frac{1}{2} \sum_{k=1}^{l} m\left[x_{k}^{-1}, y_{k}\right] x_{k} \mathbf{e}_{f, k} \otimes E_{1 n}^{(k)}(\mathbf{v}) \\
& -\frac{\kappa d}{2} \sum_{\substack{j, k=1 \\
j \neq k}}^{l} m x_{k}^{-1} \sigma_{j k} \mathbf{e}_{f, k} \mathbf{e}_{f, j} \otimes H_{1}^{(j)} E_{1 n}^{(k)}(\mathbf{v}) \\
& =\sum_{k=1}^{l} m y_{k} \mathbf{e}_{f, k} \otimes E_{1 n}^{(k)}(\mathbf{v}) \\
& +\frac{1}{2} \sum_{k=1}^{l} m\left(t x_{k}^{-1}+\kappa \sum_{\substack{j=1 \\
j \neq k}}^{l} \sum_{i=0}^{d-1} x_{k}^{-1} \xi_{k}^{-i} \sigma_{j k} \xi_{k}^{i}\right. \\
& \left.+\sum_{a=1}^{d-1} d c_{a} x_{k}^{-1}\left(\mathbf{e}_{a, k}-\mathbf{e}_{a-1, k}\right)\right) \mathbf{e}_{f, k} \otimes E_{1 n}^{(k)}(\mathbf{v}) \\
& -\frac{\kappa d}{2} \sum_{\substack{j, k=1 \\
j \neq k}}^{l} m x_{k}^{-1} \mathbf{e}_{f, k} \mathbf{e}_{f, j} \otimes\left(E_{11}^{(j)} E_{1 n}^{(k)}-E_{12}^{(j)} E_{2 n}^{(k)}\right)(\mathbf{v}) \\
& =\sum_{k=1}^{l} m\left(y_{k}+\frac{t}{2} x_{k}^{-1}\right) \mathbf{e}_{f, k} \otimes E_{1 n}^{(k)}(\mathbf{v})+\frac{d}{2} \sum_{k=1}^{l} m x_{k}^{-1}\left(c_{f}-c_{f+1}\right) \mathbf{e}_{f, k} \otimes E_{1 n}^{(k)}(\mathbf{v}) \\
& +\frac{\kappa d}{2} \sum_{\substack{j, k=1 \\
j \neq k}}^{l} m x_{k}^{-1} \mathbf{e}_{f, k} \mathbf{e}_{f, j} \sigma_{j k} \otimes E_{1 n}^{(k)}(\mathbf{v}) \\
& -\frac{\kappa d}{2} \sum_{j \neq k} m x_{k}^{-1} \mathbf{e}_{f, k} \mathbf{e}_{f, j} \otimes\left(E_{11}^{(j)} E_{1 n}^{(k)}-E_{12}^{(j)} E_{2 n}^{(k)}\right)(\mathbf{v}) \\
& =\sum_{k=1}^{l} m\left(y_{k}+\frac{t}{2} x_{k}^{-1}\right) \mathbf{e}_{f, k} \otimes E_{1 n}^{(k)}(\mathbf{v})+\kappa d \sum_{\substack{j, k=1 \\
j \neq k}}^{l} m x_{k}^{-1} \mathbf{e}_{f, k} \mathbf{e}_{f, j} \otimes E_{12}^{(j)} E_{2 n}^{(k)}(\mathbf{v}) \\
& +\frac{\kappa d}{2} \sum_{\substack{j, k=1 \\
j \neq k}}^{l} \sum_{a=3}^{n} m x_{k}^{-1} \mathbf{e}_{f, k} \mathbf{e}_{f, j} \otimes E_{1 a}^{(j)} E_{a n}^{(k)}(\mathbf{v})+\frac{d}{2} \sum_{k=1}^{l} m x_{k}^{-1}\left(c_{f}-c_{f+1}\right) \mathbf{e}_{f, k} \otimes E_{1 n}^{(k)}(\mathbf{v}) .
\end{aligned}
$$


We compute that $\left[\nu_{1, \mathbf{f}}, X_{0, \mathbf{f}-\mathbf{1}}^{-}\right](m \otimes \mathbf{v})$ equals

$$
\begin{aligned}
& \frac{1}{2} \sum_{i=3}^{n} \sum_{\substack{j, k=1 \\
j \neq k}}^{l} m x_{k}^{-1} \mathbf{e}_{f, j} \mathbf{e}_{f, k} \otimes E_{1 i}^{(j)} E_{i n}^{(k)}(\mathbf{v})+\frac{n-2}{4} \sum_{k=1}^{l} m x_{k}^{-1} \mathbf{e}_{f, k} \otimes E_{1 n}^{(k)}(\mathbf{v}) \\
& +\sum_{\substack{j, k=1 \\
j \neq k}}^{l} m x_{k}^{-1} \mathbf{e}_{f, j} \mathbf{e}_{f, k} \otimes E_{12}^{(j)} E_{2 n}^{(k)}(\mathbf{v})-\sum_{\substack{j, k=1 \\
j \neq k}}^{l} x_{k}^{-1} \mathbf{e}_{f, j} \mathbf{e}_{f, k} \otimes H_{1}^{(j)} E_{1 n}^{(k)}(\mathbf{v}) .
\end{aligned}
$$

Combining all these computations and using relations (76),(74) along with the assumption that $\lambda=\kappa d, \beta_{f}=\frac{t}{2}+\frac{d\left(c_{f}-c_{f+1}\right)}{2}-\frac{\lambda(n-2)}{4}$, we find that $X_{0,1, \mathbf{f}-\mathbf{1}}^{-}(m \otimes$ $\mathbf{v})=\sum_{k=1}^{l} m y_{k} \mathbf{e}_{f, k} \otimes E_{1 n}^{(k)}=\sum_{k=1}^{l} m \mathbf{e}_{f-1, k} y_{k} \otimes E_{1 n}^{(k)}$.

We have the following analog of theorem 8.4 in Gu1].

Theorem 9.2. Suppose that $\lambda=\kappa d$ and $\beta_{j}=\frac{t}{2}-\frac{\lambda(n-2)}{4}+\frac{\left(c_{j}-c_{j+1}\right) d}{2}$ (where $\left.c_{0}=0\right)$. There exists a functor $\mathrm{SW}: \mathrm{H}_{t, \mathbf{c}}\left(\Gamma_{l}\right)-\bmod _{R} \longrightarrow \mathbb{L}_{\lambda, \underline{\beta}}^{d}-\bmod _{L}^{l, \text { int }}$ which is given by $\operatorname{SW}(M)=M \otimes_{\mathbb{C}\left[S_{l}\right]}\left(\mathbb{C}^{n}\right)^{\otimes l}$. This functor is an equivalence of categories if $l+2<n$.

Proof. That the functor SW is well defined is a consequence of the calculations above concerning the action of $X_{0,1, \mathrm{f}}^{-}$on $\mathrm{V}^{l}$. The rest of the proof follows the same lines as the proof of Theorem 9.1 .

Theorem 9.3. Suppose that $\widetilde{\lambda}=-\frac{2 \lambda}{d}, \widetilde{b}_{\xi_{j}}=-\frac{2}{d} \sum_{k=0}^{d-1} \zeta^{j k}\left(\beta_{k}-\frac{\lambda}{2}\right), \widetilde{\mathbf{b}}=\left(\widetilde{\lambda}, \widetilde{b}_{\xi_{1}}\right.$, $\left.\ldots, \widetilde{b}_{\xi_{d-1}}\right)$ and $\widetilde{\beta}=-\frac{1}{d} \sum_{j=0}^{d-1}\left(2 \beta_{j}-\lambda\right)+\widetilde{\lambda}=-\frac{2}{d} \sum_{j=0}^{d-1} \beta_{j}+\frac{3 \lambda}{d}$. Then the algebras $\mathbb{L}_{\lambda, \underline{\beta}}^{d}$ and $\mathrm{D}_{\widetilde{\beta}, \widetilde{\mathbf{b}}}^{n}(\mathbb{Z} / d \mathbb{Z})$ are isomorphic.

This is a generalization of theorem 10.1 in Gu2].

Proof. Our strategy is to construct an epimorphism $\pi: \mathrm{D}_{\widetilde{\beta}, \tilde{\mathbf{b}}}^{n}(\mathbb{Z} / d \mathbb{Z}) \rightarrow \mathbb{L}_{\lambda, \underline{\beta}}^{d}$ and to use Corollary 8.1 to show that it is injective. We start by observing that we have a homomorphism $\pi: \tilde{\mathfrak{g l}}_{n}(\mathbb{C}[\mathbb{Z} / d \mathbb{Z}]) \longrightarrow \mathbb{L}_{\lambda, \underline{\beta}}^{d}$ which allows us to define unambiguously elements $E_{i j}(\gamma), E_{i j, \mathbf{f}} \in \mathbb{L}_{\lambda, \beta}^{d}$ as images of the corresponding elements in $\widetilde{\mathfrak{g l}}_{n}(\mathbb{C}[\mathbb{Z} / d \mathbb{Z}])$. In particular, $E_{i j, \mathbf{f}}=\frac{1}{d} \sum_{k=0}^{d-1} \zeta^{f k} E_{i j}\left(\xi^{k}\right), H_{i, \mathbf{f}}=$ $\frac{1}{d} H_{i}(1)+\frac{1}{d} \sum_{k=1}^{d-1} \zeta^{f k}\left(E_{i i}\left(\xi^{k}\right)-E_{i+1, i+1}\left(\xi^{k}\right)\right)$ for $1 \leq i \leq n-1$ and $H_{0, \mathbf{f}}=$ $\frac{1}{d} \sum_{k=1}^{d-1} \zeta^{f k}\left(E_{n n}\left(\xi^{k}\right)-\zeta^{k} E_{11}\left(\xi^{k}\right)\right)+\frac{1}{d}\left(E_{n n}(1)-E_{11}(1)\right)$.

To extend $\pi$, we start by setting $\pi\left(\mathrm{E}_{n 1}(u)\right)=\sum_{j=0}^{d-1} X_{0, \mathrm{j}}^{+}, \pi\left(\mathrm{E}_{1, n-1}(v)\right)=$ $\sum_{k=0}^{d-1}\left[X_{0,1, \mathbf{k}}^{-}, X_{n-1, \mathbf{k}}^{-}\right]$and $\pi\left(\mathrm{E}_{n 2}(u)\right)=\sum_{j=0}^{d-1}\left[X_{0, \mathbf{j}}^{+}, X_{1, \mathbf{j}}^{+}\right], \quad \pi\left(\mathrm{E}_{2, n-1}(v)\right)=$ $\sum_{k=0}^{d-1}\left[\left[X_{1, \mathbf{k}+\mathbf{1}}^{-}, X_{0,1, \mathbf{k}}^{-}\right], X_{n-1, \mathbf{k}}^{-}\right]$. Then we compute that $\left[\pi\left(\mathrm{E}_{n 2}(u)\right), \pi\left(\mathrm{E}_{2, n-1}(v)\right)\right]$ equals 


$$
\begin{aligned}
& \sum_{j, k=0}^{d-1}\left[\left[X_{0, \mathbf{j}}^{+}, X_{1, \mathbf{j}}^{+}\right],\left[\left[X_{1, \mathbf{k}+\mathbf{1}}^{-}, X_{0,1, \mathbf{k}}^{-}\right], X_{n-1, \mathbf{k}}^{-}\right]\right] \\
= & \sum_{k=0}^{d-1}\left[\left[\left[X_{0, \mathbf{k}+\mathbf{1}}^{+}, X_{1, \mathbf{k}+\mathbf{1}}^{+}\right],\left[X_{1, \mathbf{k}+\mathbf{1}}^{-}, X_{0,1, \mathbf{k}}^{-}\right]\right], X_{n-1, \mathbf{k}}^{-}\right] \\
= & \sum_{k=0}^{d-1}\left[\left[X_{0, \mathbf{k}+\mathbf{1}}^{+}, X_{0,1, \mathbf{k}}^{-}\right], X_{n-1, \mathbf{k}}^{-}\right]+\left[\left[X_{1, \mathbf{k}+\mathbf{1}}^{-},\left[H_{0,1, \mathbf{k}}, X_{1, \mathbf{k}+\mathbf{1}}^{+}\right]\right], X_{n-1, \mathbf{k}}^{-}\right] \\
= & \sum_{k=0}^{d-1}\left(\left[H_{0,1, \mathbf{k}}, X_{n-1, \mathbf{k}}^{-}\right]+\left[\left[X_{1, \mathbf{k}+\mathbf{1}}^{-},-X_{1,1, \mathbf{k}+\mathbf{1}}^{+}\right.\right.\right. \\
\quad & \left.\left.\left.\quad+\left(\beta_{k+1}-\lambda\right) X_{1, \mathbf{k}+\mathbf{1}}^{+} H_{0, \mathbf{k}}-\beta_{k+1} H_{0, \mathbf{k}} X_{1, \mathbf{k}+\mathbf{1}}^{+}\right], X_{n-1, \mathbf{k}}^{-}\right]\right) \\
= & \sum_{k=0}^{d-1}\left(X_{n-1,1, \mathbf{k}}^{-}+\beta_{k} H_{0, \mathbf{k}} X_{n-1, \mathbf{k}}^{-}-\left(\beta_{k}-\lambda\right) X_{n-1, \mathbf{k}}^{-} H_{0, \mathbf{k}}\right) \\
+ & \sum_{k=0}^{d-1}\left(\left[\left(\lambda-\beta_{k+1}\right) H_{1, \mathbf{k}+\mathbf{1}} H_{0, \mathbf{k}}+\beta_{k+1} H_{0, \mathbf{k}} H_{1, \mathbf{k}+\mathbf{1}}, X_{n-1, \mathbf{k}}^{-}\right]\right) \\
= & \sum_{k=0}^{d-1}\left(X_{n-1,1, \mathbf{k}}^{-}+\left(\beta_{k}-\frac{\lambda}{2}\right) X_{n-1, \mathbf{k}}^{-}+\frac{\lambda}{2} S\left(X_{n-1, \mathbf{k}}^{-}, H_{0, \mathbf{k}}\right)\right)+\sum_{k=0}^{d-1} \lambda H_{1, \mathbf{k}+\mathbf{1}} X_{n-1, \mathbf{k}}^{-} .
\end{aligned}
$$

We set $\pi\left(\mathrm{E}_{n 1}(v)\right)=-\frac{1}{2} \sum_{i, j, k=0}^{d-1}\left[\left[X_{0,1, \mathbf{i}}^{-}, E_{n 1, \mathbf{j}}\right], E_{n 1, \mathbf{k}}\right]$, where the element $E_{n 1, \mathbf{k}}$ is given by $E_{n 1, \mathbf{k}}=\left[\left[\cdots\left[X_{n-1, \mathbf{k}}^{-}, X_{n-2, \mathbf{k}}^{-}\right], \cdots\right], X_{1, \mathbf{k}}^{-}\right]$. Then

$$
\begin{aligned}
\pi\left(\mathrm{E}_{n 1}(v)\right)=- & \sum_{j=0}^{d-1}\left(\left[\left[X_{0,1, \mathbf{j}}^{-}, E_{n 1, \mathbf{j}+\mathbf{1}}\right], E_{n 1, \mathbf{j}}\right]\right. \\
& \left.+\frac{1}{2}\left[\left[X_{0,1, \mathbf{j}}^{-}, E_{n 1, \mathbf{j}}\right], E_{n 1, \mathbf{j}}\right]+\frac{1}{2}\left[\left[X_{0,1, \mathbf{j}}^{-}, E_{n 1, \mathbf{j}+\mathbf{1}}\right], E_{n 1, \mathbf{j}+\mathbf{1}}\right]\right) .
\end{aligned}
$$

We also set $\pi\left(\mathrm{E}_{1, n-1}(u)\right)=\sum_{j, k=0}^{d-1}\left[E_{1 n, \mathbf{j}},\left[X_{0, \mathbf{k}}^{+}, E_{1, n-1, \mathbf{k}}\right]\right]$, where the elements are simply given by $E_{1, n-1, \mathbf{k}}=\left[X_{1, \mathbf{k}}^{+},\left[\cdots,\left[X_{n-3, \mathbf{k}}^{+}, X_{n-2, \mathbf{k}}^{+}\right] \cdots\right]\right]$ and $E_{1 n, \mathbf{j}}=$ $\left[X_{1, \mathbf{j}}^{+},\left[\cdots,\left[X_{n-2, \mathbf{j}}^{+}, X_{n-1, \mathbf{j}}^{+}\right] \cdots\right]\right]$. Then

$$
\begin{aligned}
\pi\left(\mathrm{E}_{1, n-1}(u)\right)=\sum_{k=0}^{d-1}( & {\left[E_{1 n, \mathbf{k}},\left[X_{0, \mathbf{k}}^{+}, E_{1, n-1, \mathbf{k}}\right]\right] } \\
& \left.+\left[E_{1 n, \mathbf{k}-\mathbf{1}},\left[X_{0, \mathbf{k}}^{+}, E_{1, n-1, \mathbf{k}}\right]\right]\right) .
\end{aligned}
$$


We have to find $\left[\pi\left(\mathrm{E}_{n 1}(v)\right), \pi\left(\mathrm{E}_{1, n-1}(u)\right)\right]$. Computing separately the commutator of each pair of sums in (116) and (117), we find that they are all equal to zero except the following one:

$$
\sum_{j, k=0}^{d-1}\left[\left[\left[X_{0,1, \mathbf{j}}^{-}, E_{n 1, \mathbf{j}+\mathbf{1}}\right], E_{n 1, \mathbf{j}}\right],\left[E_{1 n, \mathbf{k}-\mathbf{1}},\left[X_{0, \mathbf{k}}^{+}, E_{1, n-1, \mathbf{k}}\right]\right]\right],
$$

which equals

$$
\begin{aligned}
& \sum_{j, k=0}^{d-1}\left[\left[\left[\left[X_{0,1, \mathbf{j}}^{-}, E_{n 1, \mathbf{j}+\mathbf{1}}\right], E_{n 1, \mathbf{j}}\right], E_{1 n, \mathbf{k}-\mathbf{1}}\right],\left[X_{0, \mathbf{k}}^{+}, E_{1, n-1, \mathbf{k}}\right]\right] \\
& +\sum_{j, k=0}^{d-1}\left[E_{1 n, \mathbf{k}-\mathbf{1}},\left[\left[\left[X_{0,1, \mathbf{j}}^{-},\left[X_{0, \mathbf{k}}^{+}, E_{1, n-1, \mathbf{k}}\right]\right], E_{n 1, \mathbf{j}+\mathbf{1}}\right], E_{n 1, \mathbf{j}}\right]\right] \\
& =\sum_{j=0}^{d-1}\left(\left[\left[\left[X_{0,1, \mathbf{j}}^{-}, E_{n 1, \mathbf{j}+\mathbf{1}}\right], H_{n 1, \mathbf{j}}\right],\left[X_{0, \mathbf{j}+\mathbf{1}}^{+}, E_{1, n-1, \mathbf{j}+\mathbf{1}}\right]\right]\right. \\
& \left.+\left[\left[\left[X_{0,1, \mathbf{j}}^{-}, H_{n 1, \mathbf{j}+\mathbf{1}}\right], E_{n 1, \mathbf{j}}\right],\left[X_{0, \mathbf{j}+\mathbf{2}}^{+}, E_{1, n-1, \mathbf{j}+\mathbf{2}}\right]\right]\right) \\
& -\sum_{j=0}^{d-1}\left[E_{1 n, \mathbf{j}},\left[\left[\left[H_{0,1, \mathbf{j}}, E_{1, n-1, \mathbf{j}+\mathbf{1}}\right], E_{n 1, \mathbf{j}+\mathbf{1}}\right], E_{n 1, \mathbf{j}}\right]\right] \\
& =\sum_{j=0}^{d-1}\left[\left[X_{0,1, \mathbf{j}}^{-}, E_{n 1, \mathbf{j}+\mathbf{1}}\right],\left[X_{0, \mathbf{j}+\mathbf{1}}^{+}, E_{1, n-1, \mathbf{j}+\mathbf{1}}\right]\right] \\
& -\sum_{j=0}^{d-1}\left[E_{1 n, \mathbf{j}},\left[\left[\left[H_{0,1, \mathbf{j}}, E_{1, n-1, \mathbf{j}+\mathbf{1}}\right], E_{n 1, \mathbf{j}+\mathbf{1}}\right], E_{n 1, \mathbf{j}}\right]\right] \\
& =\sum_{j=0}^{d-1}\left(\left[\left[-H_{0,1, \mathbf{j}}, E_{1, n-1, \mathbf{j}+\mathbf{1}}\right], E_{n 1, \mathbf{j}+\mathbf{1}}\right]+\left[E_{1 n, \mathbf{j}},\left[\left[\left[X_{1,1, \mathbf{j}+\mathbf{1}}^{+}, E_{2, n-1, \mathbf{j}+\mathbf{1}}\right]\right.\right.\right.\right. \\
& \left.\left.\left.\left.-\left(\beta_{j+1}-\lambda\right) E_{1, n-1, \mathbf{j}+\mathbf{1}} H_{0, \mathbf{j}}+\beta_{j+1} H_{0, \mathbf{j}} E_{1, n-1, \mathbf{j}+\mathbf{1}}, E_{n 1, \mathbf{j}+\mathbf{1}}\right], E_{n 1, \mathbf{j}}\right]\right]\right) \\
& =\sum_{j=0}^{d-1}\left(\left[\left[X_{1,1, \mathbf{j}+\mathbf{1}}^{+}, E_{2, n-1, \mathbf{j}+\mathbf{1}}\right], E_{n 1, \mathbf{j}+\mathbf{1}}\right]+\left[\lambda E_{1, n-1, \mathbf{j}+\mathbf{1}} H_{0, \mathbf{j}}+\beta_{j+1}\left[H_{0, \mathbf{j}},\right.\right.\right. \\
& \left.\left[\left[E_{1, n-1, \mathbf{j}+\mathbf{1}}\right], E_{n 1, \mathbf{j}+\mathbf{1}}\right]+\left[E_{1 n, \mathbf{j}},-\lambda E_{n, n-1, \mathbf{j}+\mathbf{1}} E_{n 1, \mathbf{j}}+\beta_{j+1}\left[E_{n, n-1, \mathbf{j}+\mathbf{1}}, E_{n 1, \mathbf{j}}\right]\right]\right) \\
& =\sum_{j=0}^{d-1}\left(-J\left(E_{n, n-1}\right)_{\mathbf{j}+\mathbf{1}}-\lambda\left[\left[\omega_{1, \mathbf{j}+\mathbf{1}}^{+}, E_{2, n-1, \mathbf{j}+\mathbf{1}}\right], E_{n 1, \mathbf{j}+\mathbf{1}}\right]+\beta_{j+1} E_{n, n-1, \mathbf{j}+\mathbf{1}}\right) \\
& +\sum_{j=0}^{d-1}\left(\lambda E_{n, n-1, \mathbf{j}+\mathbf{1}}\left(H_{n 1, \mathbf{j}}-H_{0, \mathbf{j}}\right)+\lambda E_{1, n-1, \mathbf{j}+\mathbf{1}} E_{n 1, \mathbf{j}+\mathbf{1}}\right) .
\end{aligned}
$$


Using (115) and (118), we conclude that $\left[\pi\left(\mathrm{E}_{n 1}(v)\right), \pi\left(\mathrm{E}_{1, n-1}(u)\right)\right]-\left[\pi\left(\mathrm{E}_{n 2}(u)\right)\right.$, $\left.\pi\left(\mathrm{E}_{2, n-1}(v)\right)\right]$ equals

$$
\begin{aligned}
& -\sum_{k=0}^{d-1}\left(X_{n-1,1, \mathbf{k}}^{-}+\left(\beta_{k}-\frac{\lambda}{2}\right) X_{n-1, \mathbf{k}}^{-}+\frac{\lambda}{2} S\left(X_{n-1, \mathbf{k}}^{-}, H_{0, \mathbf{k}}\right)\right) \\
& \quad+\sum_{k=0}^{d-1}\left(-\frac{\lambda}{2} S\left(H_{1, \mathbf{k}+\mathbf{1}}, X_{n-1, \mathbf{k}}^{-}\right)+J\left(E_{n, n-1}\right)_{\mathbf{k}}-\left(\beta_{k}-\frac{\lambda}{2}\right) E_{n, n-1, \mathbf{k}}\right) \\
& \quad-\frac{\lambda}{4} \sum_{k=0}^{d-1}\left(\sum_{j=3}^{n-2} S\left(E_{j, n-1, \mathbf{k}}, E_{n j, \mathbf{k}}\right)+S\left(H_{n-1,2, \mathbf{k}}, E_{n, n-1, \mathbf{k}}\right)+S\left(E_{n, n-1, \mathbf{k}}, H_{n 1, \mathbf{k}}\right)\right) \\
& \quad+\frac{\lambda}{4} \sum_{k=0}^{d-1}\left(S\left(E_{n, n-1, \mathbf{k}}, H_{1, \mathbf{k}}\right)+S\left(E_{1, n-1, \mathbf{k}}, E_{n 1, \mathbf{k}}\right)-S\left(E_{n 2, \mathbf{k}}, E_{2, n-1, \mathbf{k}}\right)\right) \\
& \quad-\frac{\lambda}{2} \sum_{k=0}^{d-1}\left(S\left(E_{n, n-1, \mathbf{k}}, H_{n 1, \mathbf{k}-1}-H_{0, \mathbf{k}-\mathbf{1}}\right)+S\left(E_{1, n-1, \mathbf{k}}, E_{n 1, \mathbf{k}}\right)\right) \\
& =-\sum_{k=0}^{d-1}\left(2\left(\beta_{k}-\frac{\lambda}{2}\right) E_{n, n-1, \mathbf{k}}+\frac{\lambda}{2 d}\left(1-\zeta^{k}\right)\left(S\left(E_{n, n-1}\left(\xi^{k}\right), E_{11}\left(\xi^{-k}\right)\right)\right.\right. \\
& \left.\quad-\frac{\lambda}{2} \sum_{k=0}^{d-1}\left(\sum_{j=1}^{n-2} S\left(E_{j, n-1, \mathbf{k}}, E_{n j, \mathbf{k}}\right)+S\left(E_{n, n-1}\left(\xi^{k}\right), E_{22}\left(\xi^{-k}\right)\right)\right)\right)
\end{aligned}
$$

The last expression is exactly what one obtains by applying $\pi$ to the right-hand side of equation (35) (except for the first term) in the case $a=n, b=1, c=$ $n-1, d=2$ for the algebra $\mathrm{D}_{\widetilde{\beta}, \tilde{\mathbf{b}}}^{n}(\mathbb{Z} / d \mathbb{Z})$.

In order to verify that $\pi$ respects relation (36), we now compute that $\left[\pi\left(\mathrm{E}_{n 1}(u)\right)\right.$, $\left.\pi\left(\mathrm{E}_{2, n-1}(v)\right)\right]$ equals

$$
\begin{aligned}
& \sum_{j, k=0}^{d-1}\left[X_{0, \mathbf{k}}^{+},\left[\left[X_{1, \mathbf{j}+\mathbf{1}}^{-}, X_{0,1, \mathbf{j}}^{-}\right], X_{n-1, \mathbf{j}}^{-}\right]\right]=\sum_{j=0}^{d-1}\left[\left[X_{1, \mathbf{j}+\mathbf{1}}^{-},\left[X_{0, \mathbf{j}+\mathbf{1}}^{+}, X_{0,1, \mathbf{j}}^{-}\right]\right], X_{n-1, \mathbf{j}}^{-}\right] \\
= & \sum_{j=0}^{d-1}\left[\left[X_{1, \mathbf{j}+\mathbf{1}}^{-}, H_{0,1, \mathbf{j}}\right], X_{n-1, \mathbf{j}}^{-}\right]=-\sum_{j=0}^{d-1}\left[X_{1,1, \mathbf{j}+\mathbf{1}}^{-}+\beta_{j+1} X_{1, \mathbf{j}+\mathbf{1}}^{-} H_{0, \mathbf{j}}\right. \\
& \left.-\left(\beta_{j+1}-\lambda\right) H_{0, \mathbf{j}} X_{1, \mathbf{j}+\mathbf{1}}^{-}, X_{n-1, \mathbf{j}}^{-}\right] \\
= & -\lambda \sum_{j=0}^{d-1} X_{n-1, \mathbf{j}}^{-} X_{1, \mathbf{j}+\mathbf{1}}^{-}=\frac{\widetilde{\lambda}}{4} \sum_{i=0}^{d-1} \zeta^{i} S\left(\pi\left(\mathrm{E}_{n, n-1}\left(\xi^{-i}\right)\right), \pi\left(\mathrm{E}_{21}\left(\xi^{i}\right)\right)\right) .
\end{aligned}
$$

The other cases of relation (35) for arbitrary $a \neq b \neq c \neq a \neq d \neq c$ and of relation (36) for arbitrary $a \neq b \neq c \neq d \neq a$ follow from the two cases above. 
Let $\Psi_{l}: \mathbb{L}_{\lambda, \underline{\beta}}^{d} \longrightarrow \operatorname{End}_{\mathbb{C}}\left(\bigvee^{l}\right)$ be the algebra map coming from the $\mathbb{L}_{\lambda, \underline{\beta}}^{d}$-module structure on $\mathrm{V}^{\bar{l}}$ given by Theorem 9.2. Then one can check that $\Psi_{l} \circ \pi=\Phi_{l}$ with $\Phi_{l}$ as defined at the end of section 8. From Corollary 8.1, we can deduce that $\pi$ must be injective.

\section{REFERENCES}

[AFLS] J. Alev, M.A. Farinati, T. Lambre, A.L. Solotar, Homologie des invariants d'une algèbre de Weyl sous l'action d'un groupe fini, J. Algebra 232 (2000), no. 2, 564-577. MR 1792746 (2002c:16047)

[ATY] S. Ariki, T. Terasoma, H. Yamada, Schur-Weyl reciprocity for the Hecke algebra of $(\mathbb{Z} / r \mathbb{Z})$ i $S_{n}$, J. Algebra 178 (1995), no. 2, 374-390. MR1359891(96k:20010)

[BEG] Y. Berest, P. Etingof, V. Ginzburg, Cherednik algebras and differential operators on quasi-invariants, Duke Math. J. 118 (2003), no. 2, 279-337. MR1980996 (2004f:16039)

[Bo] D. Boyachenko, Quantization of minimal resolutions of Kleinian singularities, Adv. Math. 211 (2007), no. 1, 244-265. MR22313534 (2008f:14001)

[CBHo] W. Crawley-Boevey, M.P. Holland, Noncommutative deformations of Kleinian singularities, Duke Math. J. 92 (1998), no. 3, 605-635. MR.1620538 (99f:14003)

[Ch1] I. Cherednik, Double affine Hecke algebras and Macdonald's conjectures, Ann. of Math. (2) 141 (1995), no. 1, 191-216. MR.1314036 (96m:33010)

[Ch2] I. Cherednik, Double affine Hecke algebras, London Mathematical Society Lecture Note Series, 319, Cambridge University Press, Cambridge, 2005. xii+434 pp. MR 2133033 (2007e:32012)

[ChPr1] V. Chari, A. Pressley, Quantum affine algebras and affine Hecke algebras, Pacific J. Math. 174 (1996), no. 2, 295-326. MR1405590 (97i:17011)

[ChPr2] V. Chari, A. Pressley, A guide to quantum groups, Cambridge University Press, Cambridge, 1994. xvi+651 pp. MR1300632 (95j:17010)

[De1] C. Dezelée, Generalized graded Hecke algebras of types B and D, Comm. Algebra 34 (2006), no. 6, 2105-2128. MR2235082 (2008b:20009)

[De2] C. Dezelée, Generalized graded Hecke algebra for complex reflection group of type $G(r, 1, n)$, arXiv:math.RT/0605410.

[Dr1] V. Drinfeld, Degenerate affine Hecke algebras and Yangians, (Russian) Funktsional. Anal. i Prilozhen. 20 (1986), no. 1, 69-70. MR831053 (87m:22044)

[Dr2] V. Drinfeld, A new realization of Yangians and of quantum affine algebras, Soviet Math. Dokl. 36 (1988), no. 2, 212-216. MR914215 (88j:17020)

[DuOp] C. Dunkl, E. Opdam, Dunkl operators for complex reflection groups, Proc. London Math. Soc. (3) 86 (2003), no. 1, 70-108. MR1971464 (2004d:20040)

[EGGO] P. Etingof, W.L. Gan, V. Ginzburg, A. Oblomkov, Harish-Chandra homomorphisms and symplectic reflection algebras for wreath-products, Publ. Math. Inst. Hautes Études Sci. No. 105 (2007), 91-155. MR2354206 (2009j:16016)

[En] B. Enriquez, $P B W$ and duality theorems for quantum groups and quantum current algebras, J. Lie Theory 13 (2003), no. 1, 21-64. MR1958574 (2004a:17016)

[EtGi] P. Etingof, V. Ginzburg, Symplectic reflection algebras, Calogero-Moser space, and deformed Harish-Chandra homomorphism, Invent. Math. 147 (2002), no. 2, 243-348. MR1881922(2003b:16021)

[Fa] M. Farinati, Hochschild duality, localization, and smash products, J. Algebra 284 (2005), no. 1, 415-434. MR2115022(2005j:16009)

[FFNR] B. Feigin, M. Finkelberg, A. Negut, L. Rybnikov, Yangians and cohomology rings of Laumon spaces, arXiv:0812.465 [math.AG].

[GaGi] W.L. Gan, V. Ginzburg, Deformed preprojective algebras and symplectic reflection algebras for wreath products, J. Algebra 283 (2005), no. 1, 350-363. MR2102087 (2005h:16049)

[GGOR] V. Ginzburg, N. Guay, E. Opdam, R. Rouquier, On the category $\mathcal{O}$ for rational Cherednik algebras, Invent. Math. 154 (2003), no. 3, 617-651. MR.2018786 (2005f:20010)

[GHL] N. Guay, D. Hernandez, S. Loktev, Double affine Lie algebras and finite groups, to appear in the Pacific Journal of Mathematics. 
[GKV] V. Ginzburg, M. Kapranov, E. Vasserot, Langlands reciprocity for algebraic surfaces, Math. Res. Lett. 2 (1995), no. 2, 147-160. MR.1324698 (96f:11086)

[GoSm] I. Gordon, P. Smith, Representations of symplectic reflection algebras and resolutions of deformations of symplectic quotient singularities, Math. Ann. 330 (2004), no. 1, 185200. MR2091684 (2006f:14013)

[GoSt] I. Gordon, J.T. Stafford, Rational Cherednik algebras and Hilbert schemes, Adv. Math. 198 (2005), no. 1, 222-274. MR2183255 (2008i:14006)

[Gu1] N. Guay, Cherednik algebras and Yangians, Int. Math. Res. Not. 2005, no.57, 3551-3593. MR2199856 (2006m:16040)

[Gu2] N. Guay, Affine Yangians and deformed double current algebras in type A, Adv. Math. 211 (2007), no. 2, 436-484. MR 2323534 (2008d:17020)

[Gu3] N. Guay, Quantum algebras and quivers, Selecta Math. (N.S.) 14 (2009), no. 3-4, 667700. MR 2511195

[He1] D. Hernandez, Representations of quantum affinizations and fusion product, Transformation Groups 10 (2005), no. 2, 163-200. MR2195598(2006k:17025)

[He2] D. Hernandez, Drinfeld coproduct, quantum fusion tensor category and applications, Proc. Lond. Math. Soc. (3) 95 (2007), no. 3, 567-608. MR2368277 (2008k:17017)

[Ka] C. Kassel, Kähler differentials and coverings of complex simple Lie algebras extended over a commutative algebra, Proceedings of the Luminy conference on algebraic $K$ theory (Luminy, 1983), J. Pure Appl. Algebra 34 (1984), no. 2-3, 265-275. MR772062 (86h:17013)

[KaLo] C. Kassel, J.L. Loday, Extensions centrales d'algèbres de Lie, Ann. Inst. Fourier (Grenoble) 32 (1982), no. 4, 119-142 (1983). MR694130 (85g:17004)

[Le1] S.Z. Levendorski, On PBW bases for Yangians, Lett. Math. Phys. 27 (1993), no. 1, 37-42. MR 1212024 (94b:17027)

[Le2] S.Z. Levendorski, On generators and defining relations of Yangians, J. Geom. Phys. 12 (1993), no. 1, 1-11. MR.1226802 (94d:17017)

[Lu] G. Lusztig, Affine Hecke algebras and their graded version, J. Amer. Math. Soc. 2 (1989), no. 3, 599-635. MR991016 (90e:16049)

[MRY] R.V. Moody, S. E. Rao, T. Yokonuma, Toroidal Lie algebras and vertex representations, Geom. Dedicata 35 (1990), no. 1-3, 283-307. MR.1066569 (91i:17032)

[RaSh] A. Ram, A. Shepler, Classification of graded Hecke algebras for complex reflection groups, Comment. Math. Helv. 78 (2003), no. 2, 308-334. MR1988199 (2004d:20007)

[VaVa1] E. Vasserot, M. Varagnolo, Schur duality in the toroidal setting, Comm. Math. Phys. 182 (1996), no. 2, 469-483. MR 1447301 (98a:17024)

[VaVa2] M. Varagnolo, E. Vasserot, Double-loop algebras and the Fock space, Invent. Math. 133 (1998), no. 1, 133-159. MR.1626481 (99g:17035)

[VaVa3] M. Varagnolo, E. Vasserot, On the K-theory of the cyclic quiver variety, Internat. Math. Res. Notices 1999, no. 18, 1005-1028. MR1722361 (2000m:14011)

[We] C. Weibel, An introduction to homological algebra, Cambridge Studies in Advanced Mathematics, 38, Cambridge University Press, Cambridge, 1994. xiv+450 pp. MR:1269324(95f:18001)

Department of Mathematical and Statistical Sciences, University of Alberta, CAB 632, Edmonton, Alberta T6G 2G1, Canada

E-mail address: nguay@math.ualberta.ca 